\title{
31. CHEMOSTRATIGRAPHY OF MADEIRA ABYSSAL PLAIN MIOCENE-PLEISTOCENE TURBIDITES, SITE 950 ${ }^{1}$
}

\author{
Ian Jarvis, ${ }^{2}$ Jennifer Moreton, ${ }^{2}$ and Martine Gérard ${ }^{3}$
}

\begin{abstract}
Miocene to Holocene sediments on the Madeira Abyssal Plain (MAP), northeast Atlantic, are dominated by thick-bedded distal mud turbidites. These turbidites record the history of sedimentary source areas and slope failure on the margins of the Canary Basin since $~ 15$ Ma. Major elements and selected trace elements have been determined in 488 turbidite samples collected between 0 and 325 mbsf (Miocene-Pleistocene) at Site 950, on the western MAP. Carbonate and Ti/Al ratio data have been plotted against a detailed sedimentary log to demonstrate the distribution of turbidite chemofacies through the succession. Three major turbidite groups, recognized previously from Quaternary cores, are confirmed to continue through the older sediment record on the plain. Organic-rich, volcanic, and calcareous turbidites are clearly differentiated on chemostratigraphic logs. Organic-rich turbidites dominate both volumetrically and numerically. They have been deposited since the middle Miocene ( 15 Ma) and may be subdivided into three geochemically distinct subgroups, the relative importance of which has changed through time. The oldest sediments are $\mathrm{Al}$ rich, reflecting more kaolinitic compositions; two $\mathrm{K}$ - and $\mathrm{Mg}$-rich subgroups become dominant upward, implying a trend toward more chloritic and illitic clay-mineral assemblages. These changes indicate an increasing importance of northerly source areas on the northwest African continental slope, and/or climatic changes promoting mineralogical shifts in sediments on the margin. The onset of significant volcanic turbidite deposition occurred in the mid-late Miocene, $\sim 14-16 \mathrm{Ma}$, with the deposition of low-Ti sediments derived from the vicinity of an evolved volcanic source, possibly the slopes of the Canary Islands off Lanzarote or Gomera. A major change toward more basaltic sources occurred in the late Pliocene ( 3.5 Ma), possibly associated with the early development of La Palma. Wide ranges in trace-element compositions and a shift toward less Ti-rich compositions indicate the continued existence of multiple sources with increasing volcanic fractionation since that time. Calcareous turbidites have been deposited regularly since the Miocene, but underwent a major decrease in their volcaniclastic component $\sim 3.5 \mathrm{Ma}$. This is interpreted to indicate the subsidence and draping of the seamount chains to the west of the MAP, which are believed to be the source area for these turbidites.
\end{abstract}

\section{INTRODUCTION}

The Madeira Abyssal Plain (MAP) lies in the deepest part of the Canary Basin at water depths of $\sim 5.4 \mathrm{~km}$. It consists of three linked sub-basins, which when combined occupy an area of approximately $68,000 \mathrm{~km}^{2}$ (Fig. 1). The morphology of the MAP is virtually flat, interrupted only by small abyssal hills that rise a few hundred meters above the plain. Quaternary sequences on the MAP represent one of the most comprehensively studied sedimentary records in the deep ocean (Weaver and Kuijpers, 1983; Colley et al., 1984, 1989; Colley and Thomson, 1985, 1992; Kuijpers and Weaver, 1985; Wilson et al., 1985, 1986; Thomson et al., 1986, 1987, 1993; Weaver et al., 1986, 1992, 1994; Weaver, Buckley et al., 1989; Weaver, Thomson, et al., 1989; De Lange et al., 1987, 1989; Jarvis and Higgs, 1987; Kidd et al., 1987; Searle, 1987; Weaver and Rothwell, 1987; Middelburg and De Lange, 1988; Pearce, 1991; De Lange, 1992a, 1992b; Jones et al., 1992; McArthur et al., 1992; Middelburg, 1993; Pearce and Jarvis 1992a, 1992b, 1995; Rothwell et al., 1992; Weaver, 1993; Weaver and Thomson, 1993).

Sediments have been recovered primarily by piston cores to a depth of up to $35 \mathrm{~m}$, representing deposition since $\sim 750 \mathrm{ka}$. More than 160 cores have been collected from the plain, sequences typically comprising meter-thick, fine-grained distal turbidites, interbedded with thin, centimeter to decimeter pelagic ooze, marl, or clay (Weaver et al., 1986; Weaver and Rothwell, 1987). Thicker turbidites may have a coarser basal facies (Rothwell et al., 1992), comprising thin

'Weaver, P.P.E., Schmincke, H.-U., Firth, J.V., and Duffield, W. (Eds.), 1998. Proc. ODP, Sci. Results, 157: College Station, TX (Ocean Drilling Program).

${ }^{2}$ School of Geological Sciences, Kingston University, Penrhyn Road, Kingston upon Thames, Surrey KT1 2EE, United Kingdom. I.Jarvis@kingston.ac.uk

${ }^{3}$ Laboratoire Petrologie et Mineralogie, Orstom, 72 Route d'Aulnay, 93143 Bondy Cedex, France. sands and silts (Units $\mathrm{T}_{\mathrm{bcd}}$ of Bouma, 1962), but these units progressively disappear distally, leaving only wispy laminated silts and muds at the base (Units $\mathrm{T}_{3-5}$ of Stow and Shanmugam, 1980). Throughout the area, the bulk of each turbidite is typically composed of $\mathrm{T}_{\mathrm{e}}\left(\mathrm{T}_{6-8}\right)$ ungraded muds.

MAP Quaternary sequences have been dated using nannofossil biostratigraphy of the pelagic marls and oozes. The turbidites them-

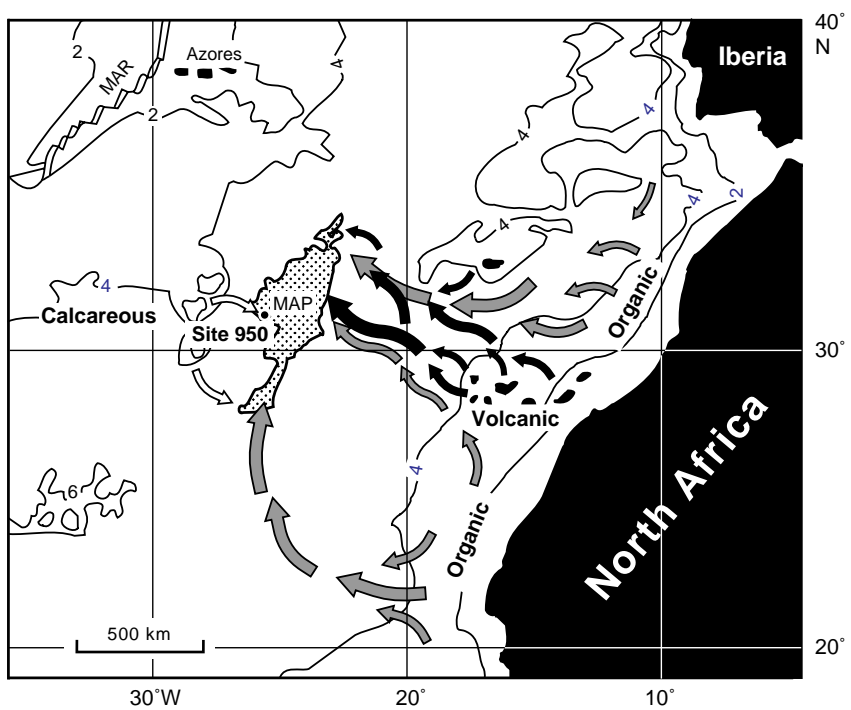

Figure 1. Location map for the Madeira Abyssal Plain (MAP) and ODP Site 950. MAR = Mid-Atlantic Ridge; bathymetry in kilometers; sediment transport pathways from Pearce and Jarvis (1995), gray arrows = organic-rich, black $=$ volcanic, white $=$ calcareous turbidites . 
selves exhibit distinctive color and thickness variation, and yield unique mixed coccolith assemblages. By combining these data, individual turbidites have been successfully correlated between cores (Weaver and Kuijpers, 1983; Weaver et al., 1986; Weaver, Buckley et al., 1989; Weaver, Thomson, et al., 1989; Weaver, 1993). In addition, De Lange et al. $(1987,1989)$ studied the bulk-geochemical analyses of turbidites from two representative MAP cores, and recognized three broad compositional groups: green organic-rich turbidites with $0.3 \%-2.5 \%$ organic carbon $\left(\mathrm{C}_{\mathrm{org}}\right)$; gray "volcanic" turbidites containing volcaniclastic debris and high concentrations of elements such as Ti; and white calcareous turbidites with high $\mathrm{CaCO}_{3}(>75 \%)$ contents. This work was extended by Pearce (1991) and Pearce and Jarvis (1992a, 1995), who used geochemical data for $>500$ samples from 23 cores, to demonstrate that individual MAP turbidites have distinctive and consistent geochemical compositions over distances of $>500 \mathrm{~km}$, which enable the chemostratigraphic correlation of sequences across the whole plain.

A combination of geochemical and sedimentological evidence (De Lange et al., 1987, 1989; Jones, 1988; McCave and Jones, 1988; Pearce, 1991; Pearce and Jarvis 1992a, 1995; Rothwell et al., 1992), mineralogical data (Weaver and Rothwell, 1987; Pearce and Jarvis, 1992b), and geological arguments (Weaver, Buckley et al., 1989; Weaver, Thomson, et al., 1989; 1992, 1994), were employed by Pearce and Jarvis (1995) to demonstrate that the turbidite compositional groups represented deposition from flows derived from five distinct source areas. Organic-rich turbidites are sourced from the northwest African continental slope (Fig. 1), one subgroup being derived from north of the Canaries, probably off the Moroccan coast; the second subgroup was derived from south of the Canaries, off Western Sahara. Volcanic turbidites originate from the slopes of the Canary Islands, one subgroup coming largely from the western islands, the other being derived from the northern flanks of the central and eastern islands. Calcareous turbidites are derived from the margins of the seamount chains lying to the west of the MAP.

Despite their young age, minimal burial, and deep-water depositional setting, MAP Quaternary turbidites display evidence of significant early diagenetic modification. Many turbidites exhibit a distinctive two-tone coloration with decimeter-thick pale gray to brown-colored tops, and thicker, darker, green lower portions. These 'bleached' tops have been produced by the post-depositional oxidation of organic matter in the upper parts of the turbidites by oxygen diffusing into the sediment from bottom water (Colley et al., 1984; Colley and Thomson, 1985; Wilson et al., 1985, 1986; Thomson et al., 1986, 1987, Chap. 32, this volume; Jarvis and Higgs, 1987; Weaver, Thomson, et al., 1989). Oxidation proceeds by the downward migration of a sharply defined redox boundary that remains active until the turbidite is cut off from its oxygen supply by the deposition of a subsequent flow, generally within a few $10 \mathrm{k} . \mathrm{y}$. The color change, which is caused by the destruction of organic matter and oxidation of reduced transitionmetal species, is particularly apparent in organic-rich $(>0.3 \%$ organic carbon) turbidites. Many elements, (As, Cd, $\mathrm{Co}, \mathrm{Cu}, \mathrm{Fe}, \mathrm{Mn}, \mathrm{Ni}, \mathrm{S}$, $\mathrm{Sb}, \mathrm{Se}, \mathrm{Tl}, \mathrm{U}, \mathrm{V}$, and Zn; Thomson et al., 1993, Chap. 32, this volume) are mobilized and relocated in response to the changing redox conditions, but it is important to note that others, particularly $\mathrm{Si}, \mathrm{Ti}$, $\mathrm{Al}, \mathrm{Mg}, \mathrm{K}$, and Zr (Jarvis and Higgs, 1987; Pearce and Jarvis 1995; Thomson et al., Chap. 32, this volume), appear to be unaffected by these early diagenetic processes.

Cores obtained during ODP Leg 157 provide the first opportunity to study the pre-late Quaternary record on the MAP. In this paper, we have applied the methods of De Lange et al. $(1987,1989)$ and Pearce and Jarvis (1992a, 1995) to sediments obtained at Site 950 to develop a chemostratigraphic framework that will be used to document the history of turbidite sedimentation on the plain since the early middle Miocene ( 15 Ma).

\section{MATERIALS}

Site 950 is located in the southwestern part of the central MAP (Fig. 1) at $31^{\circ} 9.01^{\prime} \mathrm{N}, 25^{\circ} 36.00^{\prime} \mathrm{W}$, at a water depth of $5438 \mathrm{~m}$. This location is only $25 \mathrm{~km}$ west-southwest of the site of a 34-m-long giant piston core, MD10, which contains a complete turbidite sequence beginning $\sim 690 \mathrm{ka}$ (isotope Stages 1 through 17). Core MD10 was geochemically characterized by De Lange et al. $(1987,1989)$ and, along with Core D10688, was the first to be used to demonstrate the application of chemostratigraphic methods to sediment provenance studies. The location of the site on the western margin of the plain ensures that it contains a relatively complete sediment record dominated by very distal facies, with little evidence of basal sand or silt development except in calcareous turbidites, which were derived from the west. These characteristics made the site the most appropriate choice for a chemostratigraphic type section. Shipboard (Schmincke, Weaver, Firth, et al., 1995) and subsequent biostratigraphic data (Howe and Sblendorio-Levy, Chap. 29, this volume) confirm that Site 950 exhibits a thick turbidite record down to the lowest middle Miocene.

An informal lithostratigraphy for Site 950 was proposed by Schmincke, Weaver, Firth, et al. (1995): Unit I (0-306 mbsf; meters below seafloor), comprises thick (decimeter to meter) turbidite muds separated by thin (centimeter) pelagic ooze, marl, or clay interbeds; Unit II (306-333 mbsf) consists of carbonate debris flows; Unit III (333-370 mbsf) is predominantly pelagic red clay, with thin carbonate-rich turbidites and zeolitic ash bands; Unit IV (370-381 mbsf) consists of volcaniclastic sandstones and siltstones. Unit I was divided (Schmincke, Weaver, Firth, et al., 1995) into Subunits Ia (0-150 mbsf) and Ib (150-306 mbsf), on the basis that pelagic intervals below 150 mbsf are clays rather than mixed lithologies (clays, clayey nannofossil mixed sediments, and nannofossil oozes). Following the work of De Lange et al. (1987, 1989) and Pearce and Jarvis (1992a, 1995), shipboard sedimentologists employed a genetic classification for MAP turbidites that included green (organic-rich), gray (volcanic-rich), and white (calcareous) turbidites. Other lithologies recognized (Schmincke, Weaver, Firth, et al., 1995) included gray-green (intermediate-type), light brown or white nannofossil clay, and calcareous turbidites with volcanic clasts.

In total, $48810-\mathrm{mL}$ sediment plug samples were collected from the mud portions of each turbidite $>20 \mathrm{~cm}$ thick from $0-325 \mathrm{mbsf}$ (Cores 157-950A-1H to 36X); samples were taken from below redox fronts and above any silty basal facies. Three equally spaced samples were collected from turbidites $>1 \mathrm{~m}$ thick, and one or two samples from thinner beds. The aim was to adequately characterize the primary geochemical composition of each major turbidite, and to use these data to develop a chemostratigraphic framework, which could be used to document changes in sediment composition and provenance through the Miocene-Pleistocene.

\section{ANALYTICAL METHODS}

Sample preparation methods are described elsewhere (Jarvis, 1992). Briefly, unwashed samples were freeze-dried, and ground by hand to a fine powder in an agate mortar and pestle. Homogenized samples were redried overnight at $65^{\circ} \mathrm{C} ; 0.250$-g subsamples were fused with $1.250 \mathrm{~g}$ of lithium metaborate $\left(\mathrm{LiBO}_{2}\right)$ at $1050^{\circ} \mathrm{C}$, and the melts dissolved in dilute $\mathrm{HNO}_{3}$. Final solutions were prepared in 250 $\mathrm{mL} 0.5 \mathrm{MHNO}_{3}$.

Geochemical data were obtained using a Jobin Yvon JY70 Plus ICP-AES at Kingston University. Analytical procedures and operating conditions are listed in Jarvis and Jarvis (1992) and Totland et al. (1992). In this study, nine major elements ( $\mathrm{Si}, \mathrm{Ti}, \mathrm{Al}, \mathrm{Fe}, \mathrm{Mn}, \mathrm{Mg}, \mathrm{Ca}$, 
$\mathrm{Na}$, and $\mathrm{K}$ ) and four trace elements $(\mathrm{Ba}, \mathrm{Cr}, \mathrm{Sr}$, and $\mathrm{Zr}$ ) were determined. Calibration of the ICP-AES was achieved using nine wellcharacterized rock reference materials (RRMs) and a procedural blank, selected to cover the range of elemental concentrations expected in samples. Data are reported as weight percent oxides for $\mathrm{Si}, \mathrm{Ti}$, $\mathrm{Al}, \mathrm{Fe}, \mathrm{Mg} \mathrm{Na}, \mathrm{K}$, and $\mathrm{P}$, and as $\mu \mathrm{g} / \mathrm{g}$ (parts per million) for $\mathrm{Ba}, \mathrm{Cr}$, $\mathrm{Sr}$, and $\mathrm{Zr}$. Calcium data are also presented as $\mathrm{CaCO}_{3}$, since the bulk of the Ca present occurs in the carbonate fraction.

Analytical precision, reproducibility, and accuracy were determined by replicate analyses of multiple digestions of four different RRMs (Table 1) analyzed on a routine basis with each batch of unknowns. Assessments were based on preparations and determinations made on a number of different days, over a period of 1 yr. Long-term reproducibility was generally better than $2 \%$ (with a short-term precision of $0.5 \%$ to $1 \%$ ) for all elements present at or above shale-like concentrations, deteriorating to $\sim 10 \%$ for elements at low concentrations in limestones. With reference to published data (Table 1), accuracy is considered generally to lie within the range of the reproducibility.

Data have been presented as absolute concentrations and as values normalized to $\mathrm{Al}$, the latter being used to exclude the masking affects of high but variable $\mathrm{CaCO}_{3}$ contents in samples. The rationale for this procedure is that biogenic $\mathrm{CaCO}_{3}$ is relatively pure and free from other elements ( $\mathrm{Mg}$ and $\mathrm{Sr}$ excepted), so differences in the bulk chemistry of the noncarbonate fraction are emphasized by relating values to the $\mathrm{Al}$ contents of samples. In the absence of any evidence for porewater advection, significant import or export of $\mathrm{Al}$ ions is considered unlikely for sediments of $50 \%-60 \%$ porosity buried to $<400 \mathrm{~m}$.

\section{RESULTS AND DISCUSSION}

\section{Chemostratigraphy}

De Lange et al. (1987, 1989), Pearce (1991), and Pearce and Jarvis (1995) demonstrated that, although their three major turbidite groups were defined using a range of sedimentological and geochemical criteria, members of each group could be distinguished using only $\mathrm{CaCO}_{3}$ and Ti/Al data. Organic-rich turbidite muds (i.e., excluding basal sands and silts) have the lowest $\mathrm{CaCO}_{3}$ contents, typically $45 \%-55 \%$ (a notable exception is turbidite $a_{l}$, with $18 \%-32 \%$ $\mathrm{CaCO}_{3}$ ), and low but constant $\mathrm{Ti} / \mathrm{Al}$ ratios of $\sim 0.05$. Volcanic turbidite muds have intermediate carbonate contents, generally $55 \%-65 \%$, and high but variable $\mathrm{Ti} / \mathrm{Al}$ ratios of $0.08-1.5$, while calcareous turbidite muds were defined as having $>75 \% \mathrm{CaCO}_{3}$, and yielded marginally higher $\mathrm{Ti} / \mathrm{Al}$ ratios than the organic-rich group, with values $\sim 0.06$.

The above criteria have been applied to turbidite geochemical data obtained from Site 950 (Table 2). Carbonate contents and Ti/Al ratios have been plotted against a detailed lithologic log for the interval 0-325 mbsf (Fig. 2), to illustrate relationships between sediment type, bedding characteristics and geochemical composition. A number of simplifications have been made in the construction of Figure 2. First, only turbidites are shown; pelagic sediments have been omitted for clarity, so beds are defined by the base of each turbidite. Secondly, the geochemical profiles assume constant or little compositional variation within beds. This has been demonstrated to be the case in the Quaternary of the MAP by Jarvis and Higgs, (1987), De Lange et al. (1987, 1989), and Pearce and Jarvis (1992a, 1995). The validity of the approach is confirmed by the close geochemical similarity of multiple samples from most beds (Fig. 2; Table 2); data are commonly within analytical error. Exceptions are generally confined to the lower parts of what must be subtly graded beds. Where possible, only unoxidized sediments from below fossil redox fronts were sampled from Site 950, so the geochemical profiles in Figure 2 reflect primary compositional variation within the sequence. Postdepositional oxidation of organic matter in the bleached tops of organic-rich turbidites leads to the dissolution of carbonate, producing characteristically stepped profiles for $\mathrm{CaCO}_{3}$ (Jarvis and Higgs, 1987; Thomson et al., Chap. 32, this volume); these tops are not represented in our data.

Table 1. Results obtained for rock reference materials used to assess analytical data quality.

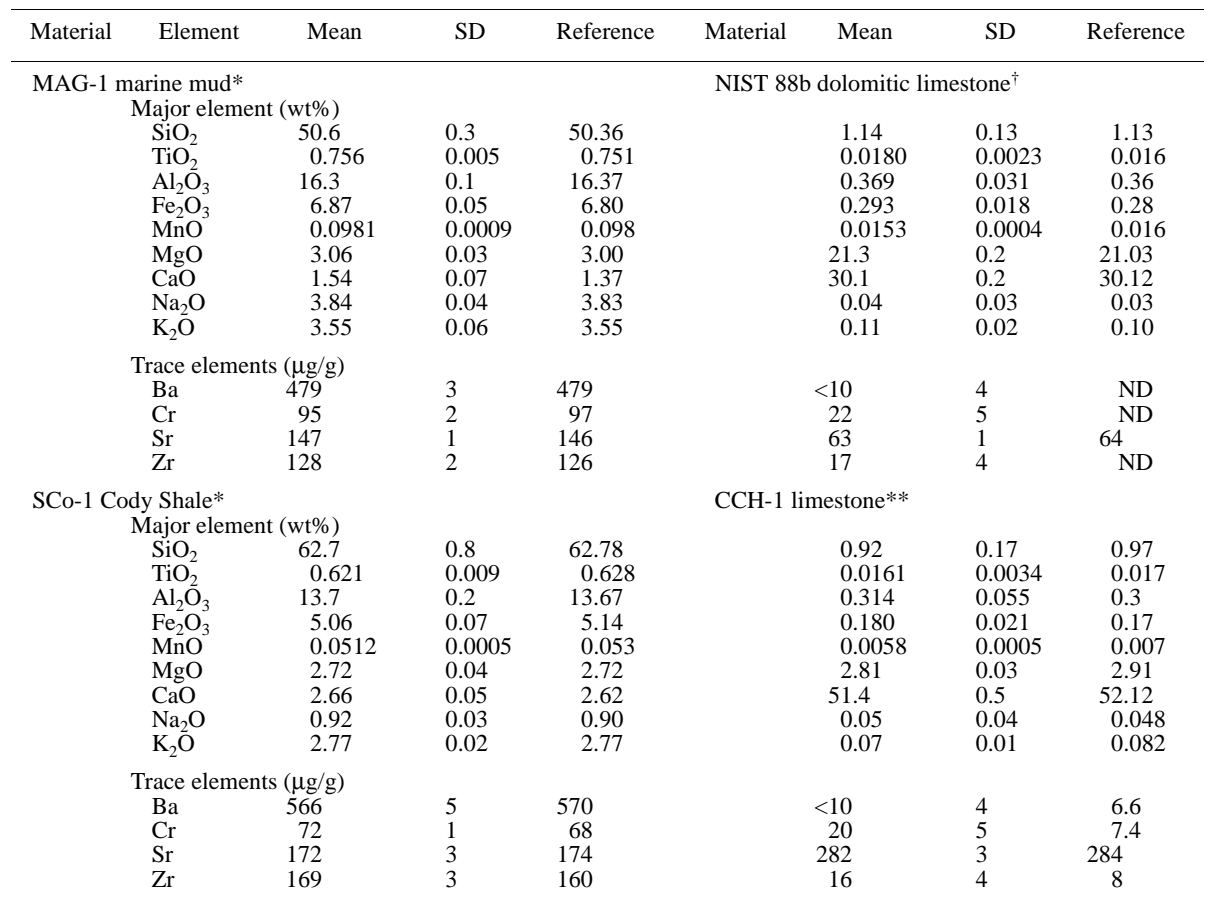

Notes: Originators: $*$ = U.S. Geological Survey; ${ }^{\dagger}=$ National Institute of Standards and Technology, U.S.A.; ** $=$ University of Liège, Belgium. Reference values from Govindaraju $(1994)$; number of determinations $=14 ; \mathrm{SD}=$ standard deviation $(\sigma \mathrm{n})$. Total iron expressed as $\mathrm{Fe}_{2} \mathrm{O}_{3} . \mathrm{ND}=$ no data available. 


\begin{tabular}{|c|c|c|c|c|c|c|c|c|c|c|c|c|c|c|c|c|c|c|}
\hline \multirow[b]{2}{*}{$\begin{array}{l}\text { Core, section, } \\
\text { interval }(\mathrm{cm})\end{array}$} & \multirow[b]{2}{*}{$\begin{array}{l}\text { Depth } \\
\text { (mbsf) }\end{array}$} & \multicolumn{9}{|c|}{ Major elements (wt\%) } & \multicolumn{4}{|c|}{ Trace elements $(\mu \mathrm{g} / \mathrm{g})$} & \multirow[b]{2}{*}{$\begin{array}{l}\mathrm{CaCO}_{3} \\
(\mathrm{wt} \%)\end{array}$} & \multirow[b]{2}{*}{$\mathrm{Ti} / \mathrm{Al}$} & & \\
\hline & & $\mathrm{SiO}_{2}$ & $\mathrm{TiO}_{2}$ & $\mathrm{Al}_{2} \mathrm{O}_{3}$ & $\mathrm{Fe}_{2} \mathrm{O}_{3}$ & $\mathrm{MnO}$ & $\mathrm{MgO}$ & $\mathrm{CaO}$ & $\mathrm{Na}_{2} \mathrm{O}$ & $\mathrm{K}_{2} \mathrm{O}$ & $\mathrm{Ba}$ & $\mathrm{Cr}$ & $\mathrm{Sr}$ & $\mathrm{Zr}$ & & & Group & Color \\
\hline 157-950A- & & & & & & & & & & & & & & & & & & \\
\hline $1 \mathrm{H}-2,63-65$ & 2.03 & 20.7 & 0.908 & 6.75 & 3.90 & 0.110 & 2.20 & 30.8 & 2.78 & 1.15 & 332 & 57 & 1150 & 124 & 55.0 & 0.152 & $\mathrm{v}$ & \\
\hline $1 \mathrm{H}-2,101-103$ & 2.41 & 20.5 & 0.873 & 6.71 & 3.82 & 0.115 & 2.17 & 31.1 & 2.93 & 1.19 & 343 & 40 & 1160 & 120 & 55.4 & 0.147 & $\mathrm{v}$ & \\
\hline $1 \mathrm{H}-2,136-138$ & 2.76 & 19.9 & 0.654 & 6.60 & 3.40 & 0.105 & 1.91 & 32.2 & 2.53 & 1.17 & 289 & 44 & 1150 & 104 & 57.5 & 0.112 & $\mathrm{v}$ & Gray $-b$ \\
\hline $1 \mathrm{H}-3,18-20$ & 3.08 & 16.6 & 0.308 & 5.45 & 2.15 & 0.0737 & 1.28 & 36.1 & 2.17 & 1.00 & 240 & 47 & 1160 & 81 & 64.3 & 0.0641 & o & ale green $-c$ \\
\hline $1 \mathrm{H}-3,113-115$ & 4.03 & 29.4 & 0.540 & 10.3 & 3.71 & 0.0683 & 2.81 & 22.5 & 2.17 & 2.08 & 408 & 86 & 780 & 98 & 40.1 & 0.0595 & $\mathrm{o}$ & Green $-d$ \\
\hline $1 \mathrm{H}-4,130-132$ & 5.70 & 21.8 & 0.433 & 7.56 & 2.76 & $\begin{array}{l}0.0003 \\
0.0621\end{array}$ & 2.06 & 30.1 & 2.42 & 1.58 & 476 & $\begin{array}{l}00 \\
49\end{array}$ & 1120 & $\begin{array}{l}90 \\
83\end{array}$ & $\begin{array}{l}40.1 \\
53.7\end{array}$ & 0.0648 & o & Green - $d$ \\
\hline $\begin{array}{l}1 \mathrm{H}-5,64-66 \\
1\end{array}$ & 6.54 & 22.1 & 0.441 & 7.38 & 2.77 & $\begin{array}{l}0.0021 \\
0.0618\end{array}$ & 2.02 & 29.5 & 2.09 & 1.44 & 474 & 48 & 1100 & $\begin{array}{l}87 \\
87\end{array}$ & 52.7 & $\begin{array}{l}0.00480 \\
0.0676\end{array}$ & o & \\
\hline $1 \mathrm{H}-6,63-65$ & 8.03 & 22.6 & 0.453 & 7.31 & 2.77 & 0.0653 & 2.04 & 29.6 & 2.35 & 1.49 & 442 & 45 & 1090 & 90 & 52.8 & 0.0703 & o & Green - $e$ \\
\hline $2 \mathrm{H}-1,79-81$ & 9.60 & 24.4 & 0.366 & 7.32 & 2.66 & 0.0471 & 1.72 & 27.1 & 2.04 & 1.17 & 582 & 60 & 993 & 79 & 48.4 & 0.0567 & o & Uicen-e \\
\hline $2 \mathrm{H}-2,79-81$ & 11.01 & 26.2 & 0.384 & 7.51 & 2.78 & 0.0501 & 1.78 & 27.4 & 2.12 & 1.24 & 563 & 72 & 1020 & 91 & 49.0 & 0.0579 & o & \\
\hline $2 \mathrm{H}-3,79-81$ & 12.49 & 25.7 & 0.374 & 7.33 & 2.80 & 0.0508 & 1.76 & 27.3 & 2.20 & 1.22 & 559 & 72 & 990 & 90 & 48.6 & 0.0578 & o & Green $-f$ \\
\hline $2 \mathrm{H}-4,98-100$ & 14.19 & 18.1 & 0.617 & 6.03 & 2.90 & 0.0949 & 1.63 & 34.0 & 2.84 & 1.12 & 306 & 36 & 1240 & 149 & 60.7 & 0.116 & $\mathrm{v}$ & \\
\hline $2 \mathrm{H}-5,104-106$ & 15.75 & 18.2 & 0.619 & 6.15 & 2.82 & 0.112 & 1.58 & 34.1 & 2.37 & 1.17 & 308 & 43 & 1240 & 150 & 60.9 & 0.114 & $\mathrm{v}$ & Gray - $g$ \\
\hline $2 \mathrm{H}-6,78-80$ & 16.98 & 25.2 & 0.429 & 8.26 & 3.02 & 0.0630 & 1.92 & 27.0 & 1.74 & 1.38 & 442 & 69 & 990 & 94 & 48.2 & 0.0588 & o & \\
\hline $2 \mathrm{H}-6,89-91$ & 17.09 & 25.3 & 0.434 & 8.31 & 3.05 & 0.0633 & 1.94 & 27.2 & 1.79 & 1.34 & 430 & 74 & 993 & 98 & 48.6 & 0.0592 & o & \\
\hline $2 \mathrm{H}-6,96-98$ & 17.16 & 24.9 & 0.432 & 8.40 & 3.05 & 0.0637 & 1.90 & 27.0 & 1.79 & 1.43 & 421 & 94 & 978 & 98 & 48.2 & 0.0583 & $\mathrm{o}$ & Green - $h$ \\
\hline $2 \mathrm{H}-7,4-6$ & 17.73 & 6.08 & 0.110 & 2.05 & 0.887 & 0.125 & 0.628 & 47.7 & 1.39 & 0.34 & 172 & 15 & 1490 & 31 & 85.1 & 0.0609 & $\mathrm{c}$ & \\
\hline $2 \mathrm{H}-7,11-13$ & 17.80 & 5.41 & 0.0975 & 1.83 & 0.794 & 0.119 & 0.651 & 48.1 & 1.88 & 0.41 & 199 & 24 & 1520 & 24 & 85.9 & 0.0604 & $\mathrm{c}$ & White $-j$ \\
\hline $2 \mathrm{H}-7,35-37$ & 18.04 & 29.2 & 0.515 & $\begin{array}{l}1.03 \\
11.6\end{array}$ & 3.95 & 0.0482 & 1.92 & $\begin{array}{l}40.1 \\
21.7\end{array}$ & $\begin{array}{l}1.00 \\
1.75\end{array}$ & $\begin{array}{l}0.41 \\
1.64\end{array}$ & 662 & 111 & 842 & 94 & $\begin{array}{l}38.8 \\
38.8\end{array}$ & 0.0503 & o & Wrinte $-j$ \\
\hline $2 \mathrm{H}-\mathrm{CC}, 10-12$ & 18.17 & 25.1 & 0.455 & 10.1 & 3.54 & 0.0534 & 1.70 & 24.6 & 1.89 & 1.35 & 550 & 90 & 933 & 86 & 43.9 & 0.0509 & o & Green - $k$ \\
\hline $3 \mathrm{H}-1,40-42$ & 18.81 & 23.8 & 0.410 & 8.67 & 3.35 & 0.0797 & 2.25 & 29.1 & 1.49 & 1.90 & 273 & 75 & 1030 & 66 & 52.0 & 0.0536 & o & Green - $l l$ \\
\hline $3 \mathrm{H}-1,98-100$ & 19.39 & 25.7 & 0.466 & 9.59 & 3.59 & 0.0783 & 2.39 & 26.8 & 1.43 & 2.18 & 273 & 60 & 948 & 78 & 47.9 & 0.0551 & o & Green - 13 \\
\hline $3 \mathrm{H}-3,23-25$ & 20.43 & 16.2 & 0.629 & 5.54 & 2.99 & 0.113 & 1.65 & 34.9 & 2.28 & 1.05 & 348 & 47 & 1260 & 90 & 62.3 & 0.129 & $\mathrm{v}$ & \\
\hline $3 \mathrm{H}-3,50-52$ & 20.70 & 16.2 & 0.575 & 5.60 & 2.87 & 0.108 & 1.62 & 35.2 & 2.20 & 1.08 & 359 & 25 & 1260 & 84 & 62.8 & 0.116 & $\mathrm{v}$ & Gray $-n$ \\
\hline $3 \mathrm{H}-4,25-27$ & 21.79 & 17.8 & 0.639 & 5.90 & 3.10 & 0.104 & 1.56 & 34.8 & 2.18 & 1.09 & 351 & 32 & 1270 & 123 & 62.1 & 0.123 & $\mathrm{v}$ & \\
\hline $3 \mathrm{H}-4,74-76$ & 22.28 & 18.7 & 0.654 & 5.89 & 3.64 & 0.110 & 1.71 & 33.2 & 2.56 & 1.13 & 348 & 52 & 1220 & 129 & 59.2 & 0.126 & $\mathrm{v}$ & \\
\hline $3 \mathrm{H}-5,12-14$ & 23.15 & 17.3 & 0.629 & 5.85 & 2.70 & 0.121 & 1.58 & 34.9 & 2.34 & 1.09 & 323 & 33 & 1270 & 118 & 62.3 & 0.122 & $\mathrm{v}$ & Gray - $o$ \\
\hline $3 \mathrm{H}-6,32-34$ & 24.85 & 16.9 & 0.755 & 5.67 & 3.05 & 0.126 & 1.81 & 35.6 & 2.65 & 0.99 & 335 & 49 & 1310 & 99 & 63.6 & 0.151 & $\mathrm{v}$ & \\
\hline $3 \mathrm{H}-6,70-72$ & 25.23 & 16.3 & 0.698 & 5.47 & 2.91 & 0.110 & 1.71 & 35.6 & 2.40 & 0.90 & 321 & 36 & 1320 & 95 & $\begin{array}{l}63.6 \\
\end{array}$ & 0.145 & $\mathrm{v}$ & Gray $-p$ \\
\hline $3 \mathrm{H}-7,42-44$ & 26.45 & 9.08 & 0.168 & 2.90 & 1.30 & 0.0633 & 0.767 & 45.0 & 1.40 & 0.64 & 197 & 3 & 1430 & 47 & 80.4 & 0.0659 & $\mathrm{c}$ & White - $q$ \\
\hline $3 \mathrm{H}-7,140-142$ & 27.43 & 5.08 & 0.100 & 1.62 & 0.70 & 0.0792 & 0.566 & 48.1 & $\begin{array}{l}1.40 \\
1.20\end{array}$ & 0.33 & 132 & 2 & 1430 & 34 & 85.9 & 0.0700 & $\mathrm{c}$ & $\begin{array}{l}\text { White }-q \\
\text { White }-r\end{array}$ \\
\hline $3 \mathrm{H}-\mathrm{CC}, 20-22$ & 27.72 & 25.6 & 0.466 & $\begin{array}{l}1.02 \\
8.44\end{array}$ & 3.18 & $\begin{array}{l}0.0742 \\
0.0841\end{array}$ & $\begin{array}{l}0.000 \\
2.42\end{array}$ & $\begin{array}{l}48.1 \\
28.1\end{array}$ & $\begin{array}{l}1.20 \\
1.73\end{array}$ & $\begin{array}{l}0.33 \\
1.91\end{array}$ & $\begin{array}{l}132 \\
629\end{array}$ & 54 & 973 & $\begin{array}{l}34 \\
92 \\
22\end{array}$ & $\begin{array}{l}5.9 \\
50.2\end{array}$ & 0.0625 & o & White - $r$ \\
\hline $4 \mathrm{H}-1,18-20$ & 28.09 & 22.9 & 0.420 & 7.56 & 3.05 & 0.0582 & 2.25 & 28.7 & 1.93 & 1.50 & 615 & 48 & 1100 & 85 & 51.3 & 0.0629 & o & Green $-s$ \\
\hline $4 \mathrm{H}-1,77-79$ & 28.66 & 28.5 & 0.512 & 10.6 & 3.44 & 0.0548 & 2.72 & 23.2 & 1.52 & 2.48 & 465 & 69 & 792 & 87 & 41.3 & 0.0547 & o & Green $-s l$ \\
\hline $4 \mathrm{H}-1,97-99$ & 28.85 & 7.55 & 0.131 & 2.30 & 0.799 & 0.0959 & 0.656 & 46.8 & 1.30 & 0.44 & 216 & 19 & 1480 & 35 & 83.6 & 0.0643 & $\mathrm{c}$ & White $-s 2$ \\
\hline $4 \mathrm{H}-1,142-144$ & 29.29 & 26.4 & 0.370 & 6.90 & 2.63 & 0.0481 & 1.92 & 27.6 & 1.86 & 1.31 & 630 & 51 & 949 & 92 & 49.3 & 0.0607 & o & Wilite -32 \\
\hline $4 \mathrm{H}-2,21-23$ & 29.57 & 26.1 & 0.359 & 6.74 & 2.49 & 0.0476 & 1.85 & 27.3 & 1.90 & 1.19 & 601 & 60 & 943 & 89 & 48.7 & 0.0603 & o & \\
\hline $4 \mathrm{H}-2,80-82$ & 30.15 & 26.3 & 0.365 & 6.70 & 2.56 & 0.0525 & 1.85 & 29.0 & 1.91 & 1.22 & 552 & 62 & 991 & 96 & 51.8 & 0.0618 & o & Green $-t$ \\
\hline $4 \mathrm{H}-3,26-28$ & 31.08 & 28.5 & 0.412 & 8.26 & 3.10 & 0.0491 & 1.95 & 24.5 & 1.86 & 1.32 & 653 & 74 & 879 & 82 & 43.8 & 0.0565 & o & \\
\hline $4 \mathrm{H}-3,66-68$ & 31.47 & 29.3 & 0.420 & 8.22 & 3.03 & 0.0515 & 1.94 & 24.6 & 1.92 & 1.31 & 615 & 76 & 882 & 93 & 43.8 & 0.0579 & $\mathrm{o}$ & Green - $u$ \\
\hline $4 \mathrm{H}-3,147-149$ & 32.26 & 24.1 & 0.323 & 6.60 & 2.49 & 0.0611 & 1.69 & 29.9 & 1.97 & 1.22 & 739 & 56 & 1090 & 66 & 53.4 & 0.0555 & o & Green \\
\hline $4 \mathrm{H}-4,66-68$ & 32.94 & 9.10 & 0.157 & 2.80 & 1.03 & 0.0879 & 0.759 & 45.0 & 1.39 & 0.52 & 195 & 18 & 1480 & 40 & 80.3 & 0.0635 & $\mathrm{c}$ & $\begin{array}{l}\text { Green } \\
\text { White }\end{array}$ \\
\hline $4 \mathrm{H}-4,112-114$ & 33.39 & 24.8 & 0.346 & $\begin{array}{l}6.94 \\
6.94\end{array}$ & 2.59 & 0.0567 & 1.72 & 28.1 & 1.90 & 1.20 & 713 & $\begin{array}{l}10 \\
54\end{array}$ & 1030 & 75 & 50.2 & 0.0565 & o & White \\
\hline $4 \mathrm{H}-5,50-52$ & 34.25 & $\begin{array}{l}25.3 \\
25.3\end{array}$ & 0.353 & $\begin{array}{l}0.94 \\
7.04\end{array}$ & 2.65 & 0.0572 & 1.72 & $\begin{array}{l}20.1 \\
28.0\end{array}$ & 1.88 & 1.25 & 719 & $\begin{array}{l}34 \\
65\end{array}$ & 1010 & $\begin{array}{l}73 \\
69\end{array}$ & 50.0 & 0.0568 & o & \\
\hline $4 \mathrm{H}-5,122-124$ & 34.95 & 25.0 & 0.356 & 7.10 & 3.44 & 0.0587 & 1.73 & 28.1 & $\begin{array}{l}1.00 \\
1.77\end{array}$ & 1.23 & 685 & 66 & 1010 & 80 & 50.2 & $\begin{array}{l}0.0508 \\
0.0569\end{array}$ & o & Green \\
\hline $4 \mathrm{H}-6,81-83$ & 36.01 & 26.8 & 0.511 & 9.03 & 3.80 & 0.0729 & 2.41 & 25.2 & 1.79 & 1.97 & 792 & 66 & 937 & 97 & 45.0 & 0.0642 & $\mathrm{o}$ & Gray \\
\hline $4 \mathrm{H}-6,129-131$ & 36.48 & 32.7 & 0.535 & 11.4 & 4.84 & 0.0607 & 2.78 & 19.7 & 1.55 & 2.41 & 537 & 92 & 666 & 86 & 35.1 & 0.0532 & o & Gray \\
\hline $4 \mathrm{H}-7,18-20$ & 36.86 & 4.59 & 0.088 & 1.58 & 0.603 & 0.0803 & 0.474 & 49.9 & 1.05 & 0.32 & 159 & 29 & 1620 & 30 & 89.0 & 0.0632 & $\mathrm{c}$ & Uiay \\
\hline $4 \mathrm{H}-7,39-41$ & 37.06 & 4.75 & 0.0908 & 1.62 & 0.648 & 0.0795 & 0.519 & 48.3 & 1.31 & 0.36 & 149 & 28 & 1580 & 29 & 86.2 & 0.0636 & $\mathrm{c}$ & White \\
\hline $5 \mathrm{H}-1,21-23$ & 37.62 & 18.9 & 0.680 & 6.21 & 3.16 & 0.105 & 1.77 & 33.5 & 2.56 & 1.20 & 335 & 39 & 1290 & 151 & 59.7 & 0.124 & $\mathrm{v}$ & \\
\hline $5 \mathrm{H}-1,55-57$ & 37.95 & 20.2 & 0.713 & 6.44 & 3.50 & 0.115 & 1.89 & 31.6 & 2.70 & 1.17 & 330 & 43 & 1250 & 152 & 56.3 & 0.126 & $\mathrm{v}$ & \\
\hline $5 \mathrm{H}-1,76-78$ & 38.15 & 18.7 & 0.626 & 5.85 & 3.48 & 0.107 & 1.85 & 31.7 & 2.81 & 1.10 & 307 & 40 & 1240 & 132 & 56.6 & 0.121 & $\mathrm{v}$ & Gray \\
\hline $5 \mathrm{H}-1,142-144$ & 38.80 & 25.9 & 0.489 & 9.20 & 3.30 & 0.0606 & 2.34 & 26.1 & 1.61 & 1.88 & 425 & 71 & 951 & 93 & 46.6 & 0.0602 & o & \\
\hline $5 \mathrm{H}-2,8-10$ & 38.96 & 25.2 & 0.480 & 8.99 & 3.27 & 0.0600 & 2.36 & 25.6 & 2.03 & 1.81 & 421 & 66 & 943 & 97 & 45.8 & 0.0605 & o & \\
\hline $5 \mathrm{H}-2,47-49$ & 39.34 & 25.2 & 0.481 & 8.91 & 3.23 & 0.0613 & 2.32 & 25.5 & 1.82 & 1.78 & 405 & 69 & 937 & 97 & 45.6 & 0.0611 & o & Pale green \\
\hline $5 \mathrm{H}-3,39-41$ & 40.72 & 17.5 & $\begin{array}{l}0.811 \\
0.844\end{array}$ & $\begin{array}{l}0.911 \\
5.57\end{array}$ & 3.55 & $\begin{array}{l}0.00106 \\
0.106\end{array}$ & 1.94 & 34.7 & $\begin{array}{l}1.02 \\
2.06\end{array}$ & $\begin{array}{l}1.10 \\
0.89\end{array}$ & 248 & 54 & 1290 & 104 & $\begin{array}{l}61.9 \\
61.9\end{array}$ & 0.172 & $\mathrm{v}$ & rale gienti \\
\hline $5 \mathrm{H}-3,117-119$ & 41.49 & 17.5 & 0.834 & 5.49 & 3.65 & 0.101 & 2.03 & 33.4 & 2.72 & 0.91 & 242 & 58 & 1240 & 103 & 59.6 & 0.172 & $\mathrm{v}$ & \\
\hline $5 \mathrm{H}$ & 42.20 & 18.6 & 0.830 & 5.70 & 3.81 & 0.0995 & 2.04 & 32.9 & 2.33 & 0. & 238 & 57 & 1230 & 106 & 58.6 & 0.165 & $\mathrm{v}$ & Gray \\
\hline $5 \mathrm{H}-4,142-144$ & 43.19 & 9.50 & 0.172 & 3.09 & 1.25 & 0.0815 & 0.759 & 44.2 & 1.0 & 0.5 & 130 & 22 & 1440 & 45 & 78.8 & 0.0629 & $\mathrm{c}$ & White \\
\hline $5 \mathrm{H}-5,72-74$ & 43.9 & 25.1 & 0.5 & 8.68 & 3.15 & 0.05 & 2.31 & 26.8 & 1.7 & 1.8 & 487 & 70 & 1000 & 93 & 47.9 & 0.0673 & o & White \\
\hline $5 \mathrm{H}-6,67-69$ & 45.39 & 25.4 & 0.520 & 8.55 & 3.10 & 0.05 & 2.29 & 27.0 & 1.94 & 1. & 466 & 76 & 1030 & 98 & 48.2 & 0.0689 & o & \\
\hline $5 \mathrm{H}-7,54-56$ & 46.73 & 26.4 & 0.543 & 8.14 & 3.05 & 0.0568 & 2.27 & 26.7 & 1.82 & 1.59 & 459 & 67 & 970 & 110 & 47.7 & 0.0756 & o & Green \\
\hline $6 \mathrm{H}-1,95-97$ & 47.84 & 29.5 & 0.530 & 11.1 & 3.75 & 0.0513 & 2.74 & 23.2 & 1.51 & 2.48 & 436 & 89 & 822 & 92 & 41.4 & 0.0541 & o & \\
\hline $6 \mathrm{H}-2,23-25$ & 48.21 & 15.9 & 0.493 & 5.31 & 2.58 & 0.0794 & 1.57 & 35.5 & 2.31 & 0.99 & 176 & 51 & 1250 & 71 & 63.4 & 0.105 & $\mathrm{v}$ & Gray \\
\hline $6 \mathrm{H}-2,84-86$ & 48.80 & 28.2 & 0.474 & 9.26 & 3.63 & 0.0404 & 2.22 & 22.8 & 2.43 & 1.54 & 551 & 81 & 873 & 97 & 40.6 & 0.0580 & o & \\
\hline
\end{tabular}


Table 2 (continued).

\begin{tabular}{|c|c|c|c|c|c|c|c|c|c|c|c|c|c|c|c|c|c|c|}
\hline \multirow[b]{2}{*}{$\begin{array}{l}\text { Core, section, } \\
\text { interval }(\mathrm{cm})\end{array}$} & \multirow[b]{2}{*}{$\begin{array}{l}\text { Depth } \\
\text { (mbsf) }\end{array}$} & \multicolumn{9}{|c|}{ Major elements (wt\%) } & \multicolumn{4}{|c|}{ Trace elements $(\mu \mathrm{g} / \mathrm{g})$} & \multirow[b]{2}{*}{$\begin{array}{l}\mathrm{CaCO}_{3} \\
(\mathrm{wt} \%)\end{array}$} & \multirow[b]{2}{*}{$\mathrm{Ti} / \mathrm{Al}$} & & \\
\hline & & $\mathrm{SiO}_{2}$ & $\mathrm{TiO}_{2}$ & $\mathrm{Al}_{2} \mathrm{O}_{3}$ & $\mathrm{Fe}_{2} \mathrm{O}_{3}$ & $\mathrm{MnO}$ & $\mathrm{MgO}$ & $\mathrm{CaO}$ & $\mathrm{Na}_{2} \mathrm{O}$ & $\mathrm{K}_{2} \mathrm{O}$ & $\mathrm{Ba}$ & $\mathrm{Cr}$ & $\mathrm{Sr}$ & $\mathrm{Zr}$ & & & Group & Color \\
\hline $6 \mathrm{H}-3,8-10$ & 49.60 & 29.2 & 0.484 & 9.45 & 3.68 & 0.0442 & 2.06 & 23.5 & 1.59 & 1.59 & 566 & 84 & 892 & 104 & 41.9 & 0.0580 & o & \\
\hline $6 \mathrm{H}-3,53-55$ & 50.04 & 29.2 & 0.483 & 9.39 & 3.70 & 0.0431 & 2.08 & 23.3 & 1.57 & 1.70 & 571 & 77 & 881 & 105 & 41.5 & 0.0583 & o & Green \\
\hline $6 \mathrm{H}-4,29-31$ & 51.27 & 22.2 & 0.388 & 7.89 & 2.73 & 0.0569 & 2.05 & 28.3 & 1.44 & 1.73 & 394 & 66 & 1030 & 73 & 50.4 & 0.0558 & o & 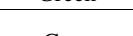 \\
\hline $6 \mathrm{H}-4,97-99$ & 51.94 & 23.1 & 0.404 & 8.04 & 2.86 & 0.0562 & 2.17 & 29.1 & 1.63 & 1.77 & 406 & 60 & 1040 & 76 & 51.9 & 0.0569 & o & Gray \\
\hline $6 \mathrm{H}-5,91-93$ & 53.35 & 19.7 & 0.481 & 7.08 & 2.70 & 0.0611 & 2.09 & 33.2 & 1.46 & 1.47 & 393 & 74 & 1240 & 88 & 59.2 & 0.0769 & o & Pale green \\
\hline $6 \mathrm{H}-6,19-21$ & 54.12 & 4.43 & 0.0741 & 1.54 & 0.460 & 0.0684 & 0.461 & 48.7 & 1.51 & 0.36 & 115 & 14 & 1620 & 27 & 86.9 & 0.0546 & $\mathrm{c}$ & White \\
\hline $6 \mathrm{H}-6,59-61$ & 54.51 & 31.6 & 0.554 & 11.7 & 4.51 & 0.0509 & 2.89 & 20.7 & 1.74 & 2.99 & 403 & 84 & 752 & 93 & 36.9 & 0.0537 & o & Gray \\
\hline $6 \mathrm{H}-6,76-78$ & 54.67 & 11.5 & 0.203 & 3.79 & 1.42 & 0.0487 & 0.901 & 42.9 & 1.31 & 0.74 & 88 & 37 & 1420 & 57 & 76.6 & 0.0608 & c & White \\
\hline 6H-CC, $13-15$ & $\begin{array}{l}55.52 \\
5580\end{array}$ & 4.56 & 0.0842 & 1.48 & $\begin{array}{l}0.583 \\
0532\end{array}$ & 0.0630 & 0.471 & 49.0 & 0.91 & 0.31 & 124 & $\begin{array}{l}35 \\
19\end{array}$ & $\begin{array}{l}1560 \\
1640\end{array}$ & 31 & 87.5 & $\begin{array}{l}0.0643 \\
0.0662\end{array}$ & $\mathrm{c}$ & White \\
\hline $6 \mathrm{H}-\mathrm{CC}, 42-44$ & 55.80 & 3.61 & 0.0725 & 1.24 & 0.538 & 0.0645 & 0.395 & 50.5 & 0.66 & 0.23 & $\begin{array}{l}121 \\
582\end{array}$ & 19 & $\begin{array}{r}1640 \\
021\end{array}$ & 24 & 90.0 & 0.0662 & c & White \\
\hline $6 \mathrm{H}-\mathrm{CC}, 95-96$ & 56.28 & 28.65 & 0.497 & 9.98 & 3.61 & 0.0380 & 2.01 & 23.8 & 1.88 & 1.51 & 583 & 103 & 921 & 103 & 42.5 & 0.0565 & o & \\
\hline $7 \mathrm{H}-1,22-24$ & 56.63 & 28.00 & 0.471 & 9.51 & 3.88 & 0.0408 & 1.88 & 23.1 & 1.66 & 1.50 & 581 & 94 & 887 & 101 & 41.3 & 0.0561 & o & \\
\hline $7 \mathrm{H}-1,37-39$ & 56.78 & 28.00 & 0.477 & 9.60 & 3.52 & 0.0434 & 1.89 & 24.6 & 1.50 & 1.45 & 580 & 100 & 937 & 102 & 44.0 & 0.0563 & o & Green \\
\hline $7 \mathrm{H}-1,98-100$ & 57.39 & 29.1 & 0.497 & 10.3 & 3.91 & 0.0674 & 2.71 & 24.3 & 1.37 & 2.45 & 387 & 82 & 871 & 83 & 43.4 & 0.0548 & o & Givin \\
\hline $7 \mathrm{H}-1,108-110$ & 57.49 & 27.8 & 0.477 & 9.84 & 3.76 & 0.0564 & 2.56 & 23.8 & 1.33 & 2.25 & 370 & 71 & 843 & 84 & 42.4 & 0.0549 & o & Gray \\
\hline $7 \mathrm{H}-1,128-130$ & 57.69 & 5.85 & 0.110 & 2.00 & 0.809 & 0.0631 & 0.577 & 48.5 & 1.05 & 0.40 & 81 & 18 & 1560 & 29 & 86.5 & 0.0623 & $\mathrm{c}$ & White \\
\hline $7 \mathrm{H}-2,67-69$ & 58.58 & 26.5 & 0.477 & 9.78 & 3.38 & 0.0464 & 2.56 & 25.7 & 1.38 & 2.42 & 365 & 77 & 919 & 83 & 45.9 & 0.0553 & o & $\begin{array}{l}\text { White } \\
\text { Green }\end{array}$ \\
\hline $7 \mathrm{H}-3,63-65$ & 60.04 & 14.1 & 0.229 & 4.60 & 1.70 & 0.0688 & 1.10 & 39.9 & 1.26 & 0.82 & 354 & 46 & 1470 & 58 & 71.2 & 0.0563 & o & Green \\
\hline $7 \mathrm{H}-4,62-64$ & 61.53 & 15.4 & 0.240 & 4.66 & 1.72 & 0.0620 & 1.14 & 36.3 & 1.53 & 0.89 & 389 & 41 & 1300 & 64 & 64.8 & 0.0583 & o & \\
\hline $7 \mathrm{H}-5,90-92$ & 63.12 & 15.3 & 0.241 & 4.76 & 1.74 & 0.0657 & 1.15 & 38.5 & 1.33 & 0.89 & 371 & 45 & 1380 & 62 & 68.8 & 0.0575 & o & \\
\hline $7 \mathrm{H}-6,52-54$ & 64.23 & 16.2 & 0.247 & 4.96 & 1.85 & 0.0615 & 1.13 & 36.6 & 1.24 & 0.96 & 432 & 46 & 1310 & 67 & 65.4 & 0.0566 & o & Gray \\
\hline $7 \mathrm{H}-7,42-44$ & 65.45 & 23.1 & 0.438 & 8.38 & 3.01 & 0.0475 & 2.11 & 30.1 & 1.34 & 1.77 & 413 & 77 & 1150 & 90 & 53.8 & 0.0593 & o & \\
\hline $7 \mathrm{H}-7,60-62$ & 65.57 & 22.4 & 0.429 & 8.16 & 2.95 & 0.0461 & 2.04 & 29.8 & 1.34 & 1.71 & 392 & 72 & 1120 & 88 & 53.2 & 0.0596 & o & Green \\
\hline $8 \mathrm{H}-1,107-109$ & 66.58 & 21.8 & 0.436 & 7.67 & 3.22 & 0.0673 & 1.95 & 31.3 & 1.32 & 1.68 & 460 & 65 & 1180 & 106 & 55.8 & 0.0644 & o & Gray \\
\hline $8 \mathrm{H}-2,81-83$ & 67.80 & 16.7 & 0.718 & 5.36 & 3.09 & 0.0740 & 1.67 & 35.6 & 2.14 & 1.05 & 215 & 65 & 1320 & 93 & 63.6 & 0.152 & $\mathrm{v}$ & Uray \\
\hline $8 \mathrm{H}-2,140-142$ & 68.39 & 15.7 & 0.615 & 5.17 & 2.77 & 0.0754 & 1.53 & 36.2 & 1.74 & 1.08 & 198 & 54 & 1320 & 81 & 64.6 & 0.135 & $\mathrm{v}$ & Gray \\
\hline $8 \mathrm{H}-3,122-124$ & 69.71 & 16.3 & 0.722 & 5.35 & 2.89 & 0.0724 & 1.69 & 34.8 & 1.88 & 1.04 & 233 & 66 & 1300 & 78 & 62.1 & 0.153 & $\mathrm{v}$ & Gray \\
\hline $8 \mathrm{H}-4,114-116$ & 71.13 & 25.0 & 0.461 & 8.63 & 2.93 & 0.0550 & 2.41 & 28.4 & 1.39 & 1.86 & 401 & 75 & 1070 & 96 & 50.6 & 0.0605 & o & Gray \\
\hline $8 \mathrm{H}-5,88-90$ & 72.36 & 25.9 & $\begin{array}{l}0.469 \\
0.401\end{array}$ & $\begin{array}{l}8.035 \\
8.35\end{array}$ & 2.91 & 0.0545 & 2.41 & 27.6 & 1.32 & 1.76 & 392 & 78 & 1020 & 108 & 49.3 & 0.0636 & o & \\
\hline $8 \mathrm{H}-6,58-60$ & 73.56 & 25.3 & 0.454 & 7.97 & 2.75 & 0.0463 & 2.30 & 27.3 & 1.35 & 1.69 & 386 & 68 & 993 & 109 & 48.6 & 0.0646 & o & Green \\
\hline $8 \mathrm{H}-7,36-38$ & 74.87 & 17.5 & 0.276 & 5.86 & 2.25 & 0.0422 & 1.50 & 35.4 & 1.37 & 1.02 & 536 & 61 & 1340 & 61 & 63.2 & 0.0534 & o & . \\
\hline 8H-CC, 3-5 & 75.15 & 17.8 & 0.276 & 5.83 & 2.26 & 0.0458 & 1.47 & 36.7 & 1.29 & 1.04 & 494 & 66 & 1390 & 62 & 65.5 & 0.0536 & o & \\
\hline $9 \mathrm{H}-1,6-8$ & 75.47 & 16.5 & 0.258 & 5.48 & 2.11 & 0.0458 & 1.40 & 35.9 & 1.36 & 1.00 & 456 & 57 & 1320 & 59 & 64.0 & 0.0534 & o & Green \\
\hline $9 \mathrm{H}-1,72-74$ & 76.12 & 27.3 & 0.493 & 10.0 & 3.75 & 0.0607 & 2.75 & 24.8 & 1.29 & 2.45 & 320 & 90 & 899 & 87 & 44.3 & 0.0556 & o & Gray \\
\hline $9 \mathrm{H}-1,117-119$ & 76.56 & 3.43 & 0.0673 & 1.12 & 0.458 & 0.0592 & 0.457 & 50.7 & 1.06 & 0.21 & 86 & 22 & 1680 & 21 & 90.5 & 0.0679 & c & White \\
\hline $9 \mathrm{H}-2,39-41$ & 77.26 & 14.4 & 0.538 & 4.73 & 2.36 & 0.0579 & 1.36 & 37.8 & 1.78 & 0.96 & 194 & 54 & 1370 & 86 & 67.5 & 0.129 & $\mathrm{v}$ & winte \\
\hline $9 \mathrm{H}-2,62-64$ & 77.49 & 12.7 & 0.487 & 4.29 & 2.11 & 0.0625 & 1.24 & 39.7 & 1.75 & 0.85 & 204 & 34 & 1470 & 73 & 70.8 & 0.129 & $\mathrm{v}$ & Gray \\
\hline $9 \mathrm{H}-2,126-128$ & 78.12 & 29.1 & 0.497 & 10.3 & 3.82 & 0.0561 & 2.84 & 23.6 & 1.23 & 2.52 & 342 & 75 & 830 & 85 & 42.1 & 0.0546 & o & Gray \\
\hline $9 \mathrm{H}-3,26-28$ & 78.61 & 4.98 & 0.0898 & 1.68 & 0.719 & 0.0503 & 0.507 & 49.4 & 0.98 & 0.33 & 62 & 12 & 1570 & 27 & 88.1 & 0.0606 & $\mathrm{c}$ & $\begin{array}{l}\text { Uray } \\
\text { White }\end{array}$ \\
\hline $9 \mathrm{H}-3,112-114$ & 79.45 & 4.13 & 0.0819 & 1.38 & 0.618 & 0.0827 & 0.464 & 50.9 & 1.01 & 0.29 & 112 & 15 & 1720 & 28 & 90.8 & 0.0675 & c & $\begin{array}{l}\text { White } \\
\text { White }\end{array}$ \\
\hline $9 \mathrm{H}-4,18-20$ & 80.00 & 18.4 & 0.890 & 5.52 & 3.93 & 0.0890 & 1.94 & 34.0 & 2.04 & 0.99 & 188 & 90 & 1300 & 101 & 60.8 & 0.183 & $\mathrm{v}$ & white \\
\hline $9 \mathrm{H}-4,95-97$ & 80.75 & 17.9 & 0.911 & 5.54 & 3.75 & 0.109 & 1.93 & 31.0 & 2.05 & 1.00 & 186 & 91 & 1200 & 96 & 55.3 & 0.186 & $\mathrm{v}$ & \\
\hline $9 \mathrm{H}-5,57-59$ & 81.85 & 17.8 & 0.887 & 5.87 & 3.23 & 0.141 & 1.82 & 32.5 & 1.97 & 1.07 & 186 & 87 & 1270 & 96 & 58.1 & 0.171 & $\mathrm{v}$ & Gray \\
\hline $9 \mathrm{H}-5,121-123$ & 82.48 & 28.0 & 0.504 & 10.4 & 3.69 & 0.0563 & 2.80 & 24.1 & 1.24 & 2.30 & 323 & 75 & 864 & 86 & 43.1 & 0.0550 & o & Hay \\
\hline $9 \mathrm{H}-5,139-141$ & 82.65 & 28.4 & 0.508 & 10.4 & 3.76 & 0.0561 & 2.83 & 23.8 & 1.26 & 2.50 & 335 & 76 & 846 & 79 & 42.4 & 0.0555 & o & Pale green \\
\hline $9 \mathrm{H}-6,66-68$ & 83.41 & 4.77 & 0.0828 & 1.56 & 0.654 & 0.0841 & 0.505 & 49.2 & 1.04 & 0.33 & 136 & 15 & 1720 & 29 & 87.8 & 0.0600 & c & White \\
\hline $9 \mathrm{H}-6,119-121$ & 83.93 & 29.7 & 0.485 & 9.79 & 3.87 & 0.0369 & 2.13 & 23.0 & 1.41 & 1.69 & 747 & 94 & 872 & 103 & 41.1 & 0.0561 & o & Wilie \\
\hline $9 \mathrm{H}-6,133-135$ & 84.06 & 29.3 & 0.481 & 9.76 & 4.09 & 0.0385 & 2.12 & 22.8 & 1.47 & 1.76 & 759 & 80 & 848 & 96 & 40.5 & 0.0558 & o & Dark green \\
\hline $9 \mathrm{H}-7,14-16$ & 84.37 & 25.3 & 0.479 & 9.66 & 3.31 & 0.0489 & 2.55 & 23.0 & 1.08 & 2.30 & 278 & 71 & 812 & 79 & 41.0 & 0.0562 & o & Green \\
\hline $9 \mathrm{H}-7,40-42$ & 84.62 & 26.2 & 0.423 & 8.73 & 3.49 & 0.0419 & 1.81 & 24.8 & 1.26 & 1.44 & 561 & 81 & 917 & 90 & 44.3 & 0.0549 & o & Ureen \\
\hline $9 \mathrm{H}-\mathrm{CC}, 8-10$ & 84.82 & 26.3 & 0.430 & 8.79 & 3.62 & 0.0461 & 1.82 & 25.7 & 1.33 & 1.50 & 582 & 82 & 951 & 95 & 45.8 & 0.0554 & o & \\
\hline $10 \mathrm{H}-1,89-91$ & 85.80 & 26.3 & 0.430 & 8.61 & 3.54 & 0.0541 & 1.79 & 26.8 & 1.42 & 1.46 & 553 & 72 & 979 & 100 & 47.9 & 0.0566 & o & Dark green \\
\hline $10 \mathrm{H}-2,42-44$ & 86.83 & 27.3 & 0.504 & 10.1 & 3.45 & 0.0555 & 2.64 & 25.1 & 1.45 & 2.17 & 379 & 77 & 970 & 94 & 44.8 & 0.0565 & o & \\
\hline $10 \mathrm{H}-2,78-80$ & 87.19 & 27.7 & 0.512 & 9.71 & 3.54 & 0.0551 & 2.57 & 24.8 & 1.23 & 2.04 & 359 & 71 & 912 & 100 & 44.3 & 0.0597 & o & Green \\
\hline $10 \mathrm{H}-4,21-23$ & 88.27 & 8.28 & 0.183 & 3.00 & 1.24 & 0.115 & 0.785 & 44.4 & 0.98 & 0.64 & 172 & 25 & 1510 & 38 & 79.3 & 0.0693 & $\mathrm{c}$ & White \\
\hline $10 \mathrm{H}-4,79-81$ & 88.85 & 16.0 & 0.709 & 5.37 & 2.87 & 0.0911 & 1.47 & 36.5 & 1.79 & 0.99 & 208 & 70 & 1360 & 85 & 65.1 & 0.150 & $\mathrm{v}$ & \\
\hline $10 \mathrm{H}-4,105-107$ & 89.11 & 16.0 & 0.686 & 5.37 & 2.89 & 0.0955 & 1.47 & 37.2 & 1.93 & 1.01 & 202 & 64 & 1350 & 85 & 66.4 & 0.145 & $\mathrm{v}$ & \\
\hline $10 \mathrm{H}-4,133-135$ & 89.36 & 15.9 & 0.657 & 5.28 & 2.78 & 0.0959 & 1.46 & 36.8 & 1.98 & 1.02 & 204 & 69 & 1370 & 83 & 65.6 & 0.141 & $\mathrm{v}$ & Pale gray \\
\hline $10 \mathrm{H}-5,138-140$ & 90.91 & 24.9 & 0.468 & 9.26 & 3.12 & 0.0477 & 2.34 & 28.9 & 1.10 & 2.08 & 370 & 69 & 1080 & 85 & 51.6 & 0.0572 & o & \\
\hline $10 \mathrm{H}-6,2-4$ & 91.05 & 24.3 & 0.458 & 8.84 & 3.01 & 0.0479 & 2.27 & 28.8 & 1.15 & 1.86 & 340 & 63 & 1070 & 84 & 51.4 & 0.0587 & o & Pale green \\
\hline $10 \mathrm{H}-6,58-60$ & 91.61 & 27.2 & 0.504 & 10.2 & 3.44 & 0.0455 & 2.72 & 25.7 & 1.33 & 2.23 & 319 & 77 & 918 & 84 & 45.9 & 0.0561 & o & Green \\
\hline $10 \mathrm{H}-6,100-102$ & 92.03 & 16.8 & 0.361 & 5.97 & 2.48 & 0.0909 & 1.49 & 33.6 & 1.04 & 1.34 & 255 & 43 & 1190 & 74 & 60.0 & 0.0686 & o & Green \\
\hline $10 \mathrm{H}-6,121-123$ & 92.24 & 17.6 & 0.388 & 6.20 & 2.71 & 0.0896 & 1.62 & 34.6 & 1.16 & 1.40 & 392 & 49 & 1250 & 79 & 61.7 & 0.0708 & o & Gray \\
\hline $10 \mathrm{H}-7,23-25$ & 92.76 & 27.4 & 0.522 & 10.3 & 3.60 & 0.0481 & 2.78 & 25.7 & 1.26 & 2.40 & 340 & 82 & 924 & 81 & 45.8 & 0.0573 & o & . \\
\hline $10 \mathrm{H}-7,32-34$ & 92.85 & 26.6 & 0.519 & 10.2 & 3.64 & 0.0502 & 2.75 & 25.7 & 1.17 & 2.40 & 344 & 83 & 925 & 78 & 45.9 & 0.0575 & o & Green \\
\hline $10 \mathrm{H}-7,81-83$ & 93.34 & 24.7 & 0.471 & 9.04 & 3.18 & 0.0583 & 2.54 & 28.0 & 1.42 & 2.00 & 318 & 71 & 1030 & 83 & 49.9 & 0.0590 & o & Sicus \\
\hline
\end{tabular}


Table 2 (continued).

\begin{tabular}{|c|c|c|c|c|c|c|c|c|c|c|c|c|c|c|c|c|c|c|}
\hline \multirow[b]{2}{*}{$\begin{array}{l}\text { Core, section, } \\
\text { interval }(\mathrm{cm})\end{array}$} & \multirow[b]{2}{*}{$\begin{array}{l}\text { Depth } \\
\text { (mbsf) }\end{array}$} & \multicolumn{9}{|c|}{ Major elements (wt\%) } & \multicolumn{4}{|c|}{ Trace elements $(\mu \mathrm{g} / \mathrm{g})$} & \multirow[b]{2}{*}{$\begin{array}{l}\mathrm{CaCO}_{3} \\
\text { (wt\%) }\end{array}$} & & & \\
\hline & & $\mathrm{SiO}_{2}$ & $\mathrm{TiO}_{2}$ & $\mathrm{Al}_{2} \mathrm{O}_{3}$ & $\mathrm{Fe}_{2} \mathrm{O}_{3}$ & $\mathrm{MnO}$ & $\mathrm{MgO}$ & $\mathrm{CaO}$ & $\mathrm{Na}_{2} \mathrm{O}$ & $\mathrm{K}_{2} \mathrm{O}$ & $\mathrm{Ba}$ & $\mathrm{Cr}$ & $\mathrm{Sr}$ & $\mathrm{Zr}$ & & $\mathrm{Ti} / \mathrm{Al}$ & Group & Color \\
\hline $10 \mathrm{H}-7,98-100$ & 93.51 & 24.9 & 0.477 & 9.16 & 3.25 & 0.0605 & 2.55 & 28.0 & 1.45 & 2.08 & 330 & 65 & 1020 & 82 & 50.0 & 0.0590 & o & \\
\hline $10 \mathrm{H}-7,124-126$ & 93.77 & 24.5 & 0.470 & 8.93 & 3.18 & 0.0602 & 2.50 & 27.5 & 1.20 & 1.91 & 323 & 68 & 1010 & 82 & 49.1 & 0.0596 & o & Green \\
\hline $11 \mathrm{H}-1,43-45$ & 94.83 & 25.2 & 0.479 & 8.56 & 3.13 & 0.0623 & 2.51 & 28.1 & 1.31 & 1.86 & 304 & 70 & 1020 & 90 & 50.1 & 0.0634 & o & \\
\hline $11 \mathrm{H}-1,112-114$ & 95.51 & 25.4 & 0.481 & 8.22 & 3.03 & 0.0643 & 2.44 & 28.6 & 1.25 & 1.72 & 307 & 66 & 1020 & 94 & 51.0 & 0.0663 & $\mathrm{o}$ & \\
\hline $11 \mathrm{H}-2,55-57$ & 96.42 & 25.7 & 0.464 & 7.82 & 2.96 & 0.0613 & 2.29 & 28.0 & 1.22 & 1.82 & 287 & 56 & 970 & 102 & 49.9 & 0.0672 & $\mathrm{o}$ & Green \\
\hline $11 \mathrm{H}-3,30-32$ & 97.65 & 2.71 & 0.0534 & 0.986 & 0.430 & 0.0647 & 0.397 & 51.5 & 0.97 & 0.17 & 68 & 15 & 1700 & 19 & 92.0 & 0.0614 & $\mathrm{c}$ & G10 \\
\hline $11 \mathrm{H}-3,60-62$ & 97.95 & 2.77 & 0.0519 & 0.958 & 0.420 & 0.0577 & 0.376 & 50.8 & 0.89 & 0.17 & 62 & 13 & 1710 & 18 & 90.6 & 0.0614 & $\mathrm{c}$ & White \\
\hline $11 \mathrm{H}-4,11-13$ & 98.94 & 27.2 & 0.544 & 10.0 & 3.62 & 0.0408 & 2.68 & 25.1 & 1.22 & 2.37 & 320 & 72 & 885 & 90 & 44.8 & 0.0616 & o & \\
\hline $11 \mathrm{H}-4,70-72$ & 99.52 & 2.92 & 0.0870 & 1.04 & 0.506 & 0.0791 & 0.390 & 51.9 & 0.88 & 0.23 & 74 & 18 & 1670 & 23 & 92.6 & 0.0952 & c & Green \\
\hline $11 \mathrm{H}-4,90-92$ & 99.71 & 3.09 & 0.0938 & 1.08 & 0.511 & 0.0849 & 0.383 & 51.0 & 0.85 & 0.21 & 75 & 16 & 1620 & 21 & 91.0 & 0.0985 & c & White \\
\hline $11 \mathrm{H}-5,8-10$ & 100.38 & 19.1 & 0.449 & 6.98 & 2.58 & 0.0515 & 1.95 & 33.4 & 1.10 & 1.50 & 370 & 57 & 1310 & 74 & 59.5 & 0.0728 & o & Green \\
\hline $11 \mathrm{H}-5,61-63$ & 100.90 & 29.6 & 0.546 & 10.9 & 3.93 & 0.0435 & 3.00 & 22.2 & 1.19 & 2.62 & 331 & 77 & 824 & 93 & 39.6 & 0.0567 & o & Green \\
\hline $11 \mathrm{H}-5,75-77$ & 101.05 & 29.3 & 0.548 & 11.0 & 3.98 & 0.0454 & 3.03 & 22.3 & 1.17 & 2.52 & 323 & 73 & 843 & 93 & 39.8 & 0.0567 & o & Green \\
\hline $11 \mathrm{H}-6,15-17$ & 101.90 & 16.8 & 0.853 & 5.71 & 3.14 & 0.102 & 1.67 & 35.1 & 1.82 & 1.08 & 185 & 101 & 1340 & 90 & 62.6 & 0.169 & $\mathrm{v}$ & \\
\hline $11 \mathrm{H}-6,50-52$ & 102.24 & 16.7 & 0.825 & 5.54 & 3.18 & 0.103 & 1.68 & 35.3 & 1.85 & 1.02 & 169 & 80 & 1330 & 86 & 62.9 & 0.169 & $\mathrm{v}$ & Gray \\
\hline $11 \mathrm{H}-6,121-123$ & 102.94 & 25.1 & 0.476 & 9.21 & 3.24 & 0.0484 & 2.66 & 26.6 & 1.07 & 2.12 & 324 & 69 & 934 & 77 & 47.5 & 0.0585 & o & Green \\
\hline $11 \mathrm{H}-7,29-31$ & 103.51 & 30.2 & 0.548 & 11.1 & 3.85 & 0.0403 & 3.00 & 22.0 & 1.27 & 2.50 & 307 & 83 & 847 & 99 & 39.2 & 0.056 & o & $\begin{array}{l}\text { Green } \\
\text { Green }\end{array}$ \\
\hline $11 \mathrm{H}-\mathrm{CC}, 4-6$ & 103.85 & 26.0 & 0.480 & 9.85 & 3.45 & 0.0452 & 2.56 & 25.9 & 1.22 & 2.19 & 350 & 65 & 973 & 83 & 46.2 & 0.0552 & o & $\begin{array}{l}\text { Green } \\
\text { Green }\end{array}$ \\
\hline $12 \mathrm{H}-1,120-122$ & 105.07 & 27.4 & 0.502 & 9.58 & 3.45 & 0.0466 & 2.67 & 25.5 & 1.11 & 2.10 & 336 & 79 & 962 & 90 & 45.4 & 0.0594 & o & Green \\
\hline $12 \mathrm{H}-2,19-21$ & 105.54 & 26.3 & 0.481 & 9.05 & 3.40 & $\begin{array}{l}0.0450 \\
0.040\end{array}$ & 2.56 & 26.4 & 1.13 & 1.99 & 318 & 75 & 976 & 90 & 47.0 & 0.0602 & 0 & Green \\
\hline $12 \mathrm{H}-2,58-60$ & 105.92 & 24.7 & 0.427 & 9.05 & 3.31 & 0.0899 & 2.86 & 28.0 & 1.01 & 2.12 & 293 & 64 & 947 & 73 & 50.0 & 0.0535 & o & Gray \\
\hline $12 \mathrm{H}-2,104-106$ & 106.37 & 20.8 & 0.457 & 7.32 & 3.02 & 0.0761 & 2.03 & 32.1 & $\begin{array}{l}1.20 \\
1.20\end{array}$ & 1.66 & 413 & $\begin{array}{l}04 \\
50\end{array}$ & $\begin{array}{r}947 \\
1150\end{array}$ & 101 & 57.3 & 0.0708 & o & Gray \\
\hline $\begin{array}{l}12 \mathrm{H}-2,-104-100 \\
1 \mathrm{H}-3,60-62\end{array}$ & 107.40 & $\begin{array}{l}24.8 \\
24.7\end{array}$ & $\begin{array}{l}0.451 \\
0.457\end{array}$ & $\begin{array}{l}1.32 \\
9.08\end{array}$ & 3.38 & $\begin{array}{l}0.0101 \\
0.0522\end{array}$ & $\begin{array}{l}2.03 \\
2.57\end{array}$ & $\begin{array}{l}32.1 \\
27.8\end{array}$ & $\begin{array}{l}1.20 \\
1.15\end{array}$ & $\begin{array}{l}1.00 \\
2.00\end{array}$ & $\begin{array}{l}413 \\
333\end{array}$ & $\begin{array}{l}50 \\
71\end{array}$ & $\begin{array}{l}1100 \\
1030\end{array}$ & $\begin{array}{r}101 \\
80\end{array}$ & $\begin{array}{l}3.3 \\
49.7\end{array}$ & $\begin{array}{l}0.0708 \\
0.0571\end{array}$ & $\begin{array}{l}0 \\
0\end{array}$ & Gray \\
\hline $12 \mathrm{H}-3,119-121$ & 107.97 & 24.2 & 0.447 & 9.05 & 3.05 & 0.0528 & 2.53 & 27.6 & 1.14 & 1.89 & 335 & 70 & 1020 & $\begin{array}{l}\text { ov } \\
78\end{array}$ & 49.2 & 0.0560 & o & \\
\hline $12 \mathrm{H}-4,30-32$ & 108.56 & 24.9 & 0.457 & 9.21 & 3.15 & 0.0573 & 2.59 & 27.9 & 1.13 & 2.04 & 334 & 78 & 1040 & 80 & 49.7 & 0.0562 & o & \\
\hline $12 \mathrm{H}-4,96-98$ & 109.20 & 26.1 & 0.471 & 8.09 & 3.10 & 0.0657 & 2.49 & 27.8 & 1.18 & 1.91 & 312 & 58 & 956 & $\begin{array}{l}80 \\
96\end{array}$ & $\begin{array}{l}49.7 \\
49.7\end{array}$ & $\begin{array}{l}0.0302 \\
0.0660\end{array}$ & $\begin{array}{l}0 \\
0\end{array}$ & \\
\hline $12 \mathrm{H}-5,26-28$ & 109.98 & 27.5 & 0.473 & 7.40 & 2.68 & 0.0642 & 2.32 & 27.2 & 1.20 & 1.77 & 302 & 47 & 904 & 105 & 48.5 & 0.0724 & o & Green \\
\hline $12 \mathrm{H}-5,90-92$ & 110.60 & 27.5 & 0.493 & 9.82 & 3.58 & 0.0506 & 2.87 & 24.6 & 1.16 & 2.38 & 347 & 75 & 877 & 80 & 43.9 & 0.0569 & o & Ginn \\
\hline $12 \mathrm{H}-5,110-112$ & 110.79 & 28.3 & 0.501 & 9.85 & 3.37 & 0.0501 & 2.90 & 24.9 & 1.14 & 2.37 & 357 & 80 & 881 & 88 & 44.5 & 0.0576 & o & Green \\
\hline $12 \mathrm{H}-6,27-29$ & 111.44 & 31.3 & 0.577 & 11.4 & 3.89 & 0.0397 & 3.03 & 21.1 & 1.17 & 2.50 & 390 & 95 & 783 & 102 & 37.6 & 0.0576 & o & Green \\
\hline $12 \mathrm{H}-6,100-102$ & 112.15 & 4.49 & 0.0939 & 1.57 & 0.632 & 0.0704 & 0.530 & 50.6 & 0.86 & 0.29 & 62 & 20 & 1610 & 24 & 90.3 & 0.0677 & $\mathrm{c}$ & Green \\
\hline $12 \mathrm{H}-7,30-32$ & 112.93 & 5.59 & 0.115 & 1.96 & 0.737 & 0.0637 & 0.593 & 49.2 & 0.89 & 0.36 & 67 & 22 & 1540 & 28 & 87.9 & 0.0663 & c & White \\
\hline $13 \mathrm{H}-1,88-90$ & 114.19 & 30.5 & 0.497 & $\begin{array}{l}1.30 \\
10.3\end{array}$ & 3.84 & 0.0314 & 2.01 & 22.4 & 1.34 & 1.59 & 464 & 110 & 878 & 113 & 40.0 & 0.0547 & o & Winte \\
\hline $13 \mathrm{H}-2,49-51$ & 115.30 & 30.1 & 0.487 & 10.1 & $\begin{array}{l}3.04 \\
3.67\end{array}$ & $\begin{array}{l}0.0314 \\
0.0315\end{array}$ & 1.98 & 22.3 & 1.34 & 1.60 & $\begin{array}{l}404 \\
461\end{array}$ & 98 & $\begin{array}{l}810 \\
862\end{array}$ & 111 & 39.7 & 0.0547 & 0 & \\
\hline $13 \mathrm{H}-2,138-140$ & $\begin{array}{l}116.30 \\
116.19\end{array}$ & 30.0 & $\begin{array}{l}0.481 \\
0.480\end{array}$ & $\begin{array}{l}10.1 \\
9.90\end{array}$ & 3.56 & $\begin{array}{l}0.0329 \\
0.0329\end{array}$ & $\begin{array}{l}1.98 \\
1.93\end{array}$ & 23.7 & $\begin{array}{l}1.04 \\
1.28\end{array}$ & $\begin{array}{l}1.00 \\
1.54\end{array}$ & $\begin{array}{l}401 \\
480\end{array}$ & $\begin{array}{l}98 \\
99\end{array}$ & $\begin{array}{l}802 \\
909\end{array}$ & 111 & 42.2 & $\begin{array}{l}0.0347 \\
0.0550\end{array}$ & $\begin{array}{l}0 \\
0\end{array}$ & \\
\hline $13 \mathrm{H}-3,81-83$ & 117.12 & 29.6 & 0.465 & 9.46 & 3.60 & 0.0389 & 1.85 & 25.3 & 1.27 & 1.52 & 471 & 99 & 945 & 115 & 45.1 & 0.0556 & o & Green \\
\hline $13 \mathrm{H}-4,4-6$ & 117.85 & 30.9 & 0.549 & 11.3 & 3.78 & 0.0584 & 2.98 & 22.1 & 1.17 & 2.51 & 322 & 89 & 821 & 112 & 39.4 & 0.0551 & o & Green \\
\hline $13 \mathrm{H}-4,52-54$ & 118.33 & 21.9 & 1.24 & 7.34 & 4.10 & 0.0964 & 2.08 & 30.8 & 2.36 & 1.25 & 157 & 47 & 1210 & 138 & 55.0 & 0.191 & $\mathrm{v}$ & Green \\
\hline $13 \mathrm{H}-4,136-138$ & 119.16 & 22.0 & 1.26 & 7.39 & 4.42 & 0.0809 & 2.17 & 28.7 & 2.39 & 1.17 & 163 & 54 & 1140 & 129 & 51.1 & 0.194 & $\mathrm{v}$ & Gray \\
\hline $13 \mathrm{H}-\mathrm{CC}, 15-17$ & 119.87 & 20.6 & 0.384 & 7.41 & 2.51 & 0.0508 & 1.87 & 33.1 & 1.04 & 1.60 & 338 & 59 & 1207 & 67 & 59.0 & 0.0588 & o & Green \\
\hline $14 \mathrm{H}-1,32-34$ & 123.23 & 27.0 & 0.494 & 9.99 & 3.79 & 0.0494 & 2.63 & 24.4 & 1.11 & 2.20 & 308 & 67 & 897 & 82 & 43.5 & 0.0560 & o & Green \\
\hline $14 \mathrm{H}-1,75-77$ & 123.66 & 28.6 & 0.517 & 10.3 & 3.84 & 0.0497 & 2.72 & 24.7 & 1.12 & 2.22 & 293 & 74 & 911 & 85 & 44.1 & 0.0570 & o & \\
\hline $14 \mathrm{H}-1,140-142$ & 124.31 & 26.4 & 0.482 & 9.38 & 3.26 & 0.0557 & 2.52 & 27.5 & 1.12 & 1.98 & 273 & 65 & 997 & 84 & 49.1 & 0.0582 & o & Green \\
\hline $14 \mathrm{H}-2,59-61$ & 125.00 & 25.7 & 0.459 & 9.26 & 3.30 & 0.0503 & 2.75 & 28.4 & 1.07 & 2.15 & 307 & 74 & 1030 & 72 & 50.7 & 0.0562 & 0 & Green \\
\hline $14 \mathrm{H}-2,137-139$ & 125.78 & 28.0 & 0.516 & 9.85 & 3.78 & 0.0494 & 2.69 & 26.2 & 1.13 & 2.13 & 321 & 75 & 972 & 93 & 46.7 & 0.0594 & o & Green \\
\hline $14 \mathrm{H}-3,77-79$ & 126.68 & 27.6 & $\begin{array}{l}0.510 \\
0.505\end{array}$ & $\begin{array}{l}.03 \\
9.42\end{array}$ & 3.50 & $\begin{array}{l}0.0494 \\
0.0507\end{array}$ & 2.60 & 25.5 & 1.06 & 2.22 & 308 & 61 & 914 & 83 & $\begin{array}{l}4.1 \\
45.4\end{array}$ & 0.0608 & 0 & \\
\hline $14 \mathrm{H}-4,10-12$ & 127.51 & $\begin{array}{l}26.0 \\
26.6\end{array}$ & 0.476 & $\begin{array}{l}. .42 \\
8.80\end{array}$ & 3.19 & $\begin{array}{l}0.0501 \\
0.0562\end{array}$ & 2.44 & 28.3 & $\begin{array}{l}1.00 \\
1.11\end{array}$ & 2.01 & $\begin{array}{l}308 \\
280\end{array}$ & $\begin{array}{l}01 \\
72\end{array}$ & $\begin{array}{r}914 \\
1020\end{array}$ & $\begin{array}{l}83 \\
96\end{array}$ & $\begin{array}{l}4.4 \\
50.5\end{array}$ & $\begin{array}{l}0.0008 \\
0.0613\end{array}$ & o & Green \\
\hline $14 \mathrm{H}-4,58-60$ & 127.99 & $\begin{array}{l}20.0 \\
15.8\end{array}$ & $\begin{array}{l}0.470 \\
0.939\end{array}$ & $\begin{array}{l}8.80 \\
5.46\end{array}$ & $\begin{array}{l}5.19 \\
3.52\end{array}$ & $\begin{array}{l}0.0502 \\
0.0942\end{array}$ & $\begin{array}{l}2.44 \\
1.64\end{array}$ & $\begin{array}{l}28.3 \\
35.7\end{array}$ & $\begin{array}{l}1.11 \\
2.07\end{array}$ & $\begin{array}{l}2.01 \\
0.93\end{array}$ & $\begin{array}{l}280 \\
117\end{array}$ & 38 & $\begin{array}{l}1020 \\
1350\end{array}$ & $\begin{array}{l}70 \\
89\end{array}$ & 63.6 & $\begin{array}{l}0.0013 \\
0.195\end{array}$ & $\mathrm{v}$ & Green \\
\hline $14 \mathrm{H}-4,90-92$ & 128.31 & 16.6 & 0.965 & 5.62 & 3.71 & 0.0978 & 1.71 & 36.7 & 2.06 & 0.91 & 114 & 50 & 1400 & 103 & 65.5 & 0.195 & $\mathrm{v}$ & Gray \\
\hline $14 \mathrm{H}-5,15-17$ & 129.06 & 14.6 & 0.721 & $\begin{array}{l}5.02 \\
5.04\end{array}$ & 2.59 & $\begin{array}{l}0.0920 \\
0.0920\end{array}$ & 1.28 & $\begin{array}{l}30.1 \\
38.8\end{array}$ & $\begin{array}{l}2.00 \\
1.73\end{array}$ & $\begin{array}{l}0.91 \\
1.02\end{array}$ & $\begin{array}{l}114 \\
195\end{array}$ & 32 & $\begin{array}{l}1400 \\
1410\end{array}$ & $\begin{array}{l}103 \\
119\end{array}$ & $\begin{array}{l}6.5 \\
69.2\end{array}$ & $\begin{array}{l}0.195 \\
0.162\end{array}$ & $\begin{array}{l}\mathrm{v} \\
\mathrm{v}\end{array}$ & Gray \\
\hline $\begin{array}{l}14 \mathrm{H}-6,7-9 \\
\text {. }\end{array}$ & 130.48 & 15.0 & 0.782 & 5.26 & 2.61 & 0.104 & 1.26 & 37.6 & 1.71 & 1.06 & 199 & 28 & 1380 & 126 & 67.1 & 0.169 & $\mathrm{v}$ & \\
\hline $14 \mathrm{H}-6,134-136$ & 131.73 & 15.4 & 0.716 & 5.22 & 2.49 & 0.105 & 1.21 & 37.9 & 1.67 & 1.01 & 194 & 51 & 1420 & 119 & 67.7 & 0.156 & $\mathrm{v}$ & Gray \\
\hline $15 \mathrm{H}-1,7-9$ & 132.48 & 22.1 & 0.356 & 7.56 & 3.56 & 0.0457 & 1.46 & 31.2 & 1.15 & 1.28 & 423 & 87 & 1170 & 79 & 55.7 & 0.0534 & o & \\
\hline $15 \mathrm{H}-1,50-52$ & 132.91 & 21.9 & 0.352 & 7.51 & 3.11 & 0.0478 & 1.42 & 31.4 & 1.13 & 1.33 & 413 & 83 & 1170 & 77 & 56.1 & 0.0531 & o & \\
\hline $15 \mathrm{H}-1,88-90$ & 133.29 & 21.3 & 0.347 & 7.35 & 2.71 & 0.0498 & 1.39 & 31.3 & 1.12 & 1.18 & 431 & 70 & 1150 & 75 & 55.8 & 0.0535 & o & Green \\
\hline $15 \mathrm{H}-1,130-132$ & 133.71 & 26.0 & 0.516 & 10.1 & 3.45 & 0.0659 & 2.84 & 25.6 & 1.12 & 2.34 & 279 & 100 & 922 & 85 & 45.7 & 0.0581 & o & Green \\
\hline $15 \mathrm{H}-2,54-55$ & 134.44 & 45.3 & 0.943 & 20.4 & 6.53 & 0.0445 & 1.70 & 4.48 & 1.36 & 2.22 & 417 & 150 & 269 & 165 & 8.00 & 0.0523 & o & Green \\
\hline $15 \mathrm{H}-2,58-59$ & 134.48 & 44.5 & 0.933 & 20.0 & 6.51 & $\begin{array}{l}0.0450 \\
0.045\end{array}$ & 1.66 & $\begin{array}{l}4.40 \\
4.34\end{array}$ & 1.34 & 2.16 & 409 & 142 & 264 & 159 & $\begin{array}{l}0.00 \\
7.74\end{array}$ & 0.0528 & o & \\
\hline $15 \mathrm{H}-2,69-70$ & $\begin{array}{l}134.48 \\
134.59\end{array}$ & 45.7 & $\begin{array}{l}0.953 \\
0.955\end{array}$ & 20.5 & $\begin{array}{l}0.01 \\
6.77\end{array}$ & $\begin{array}{l}0.0450 \\
0.0462\end{array}$ & $\begin{array}{l}1.00 \\
1.69\end{array}$ & $\begin{array}{l}4.34 \\
4.34\end{array}$ & $\begin{array}{l}1.04 \\
1.33\end{array}$ & 2.21 & 397 & 149 & $\begin{array}{l}204 \\
264\end{array}$ & 163 & 7.75 & $\begin{array}{l}0.0328 \\
0.0529\end{array}$ & $\begin{array}{l}0 \\
0\end{array}$ & Dark green \\
\hline $15 \mathrm{H}-2,120-122$ & 135.11 & 4.13 & 0.0911 & 1.54 & $\begin{array}{l}0.711 \\
0.729\end{array}$ & $\begin{array}{l}0.0402 \\
0.0869\end{array}$ & $\begin{array}{l}1.09 \\
0.454\end{array}$ & 50.3 & $\begin{array}{l}1.33 \\
0.88\end{array}$ & 0.30 & $\begin{array}{r}391 \\
32\end{array}$ & $\begin{array}{r}149 \\
19\end{array}$ & $\begin{array}{r}204 \\
1600\end{array}$ & $\begin{array}{r}103 \\
23\end{array}$ & 89.8 & $\begin{array}{l}0.0329 \\
0.0671\end{array}$ & $\begin{array}{l}0 \\
\mathrm{c}\end{array}$ & White \\
\hline $15 \mathrm{H}-3,4-6$ & 135.45 & 4.52 & 0.115 & 1.52 & 0.761 & 0.0924 & 0.473 & 50.7 & 0.67 & 0.29 & 43 & 18 & 1590 & 24 & 90.5 & 0.0855 & c & $\begin{array}{l}\text { White } \\
\text { White }\end{array}$ \\
\hline $15 \mathrm{H}-3,62-64$ & 136.03 & 25.9 & 0.485 & 9.12 & 3.26 & 0.0502 & 2.82 & 25.3 & 1.07 & 2.18 & 267 & 70 & 873 & 80 & 45.1 & 0.0603 & 0 & White \\
\hline $0-72$ & 136.11 & 25.8 & 0.488 & 9.34 & 3.26 & 0.0501 & 2.81 & 26.0 & 1.05 & 2.19 & 26 & 82 & 904 & 81 & 46.4 & 0.0592 & o & Green \\
\hline $15 \mathrm{H}-4,51-53$ & 137 & 23.8 & $\begin{array}{l}0.400 \\
1.12\end{array}$ & 8.42 & 4.62 & 0.122 & 2. & 27.8 & 1.90 & 1.78 & 29 & $\begin{array}{l}02 \\
55\end{array}$ & $\begin{array}{r}304 \\
1100\end{array}$ & 151 & $\begin{array}{l}40.4 \\
49.5\end{array}$ & & $\mathrm{v}$ & \\
\hline $15 \mathrm{H}-4,62-64$ & 137.53 & 23.5 & 1.09 & 8.35 & 4. & 0.131 & 2. & 29.0 & 1.8 & 1.76 & 292 & 56 & 1140 & 147 & 51.8 & 0.148 & $\mathrm{v}$ & Gray \\
\hline $15 \mathrm{H}-4,95-97$ & 137.86 & 27.9 & 0.528 & 10.6 & 3.63 & 0.0546 & 2.99 & 24.0 & 1.04 & 2.51 & 279 & 90 & 841 & 88 & 42.9 & 0.0567 & o & Dark o \\
\hline $15 \mathrm{H}-5,114-116$ & 139.55 & 23.8 & 0.658 & 8.94 & 3.39 & 0.0918 & 2.02 & 29.2 & 1.34 & 1.91 & 281 & 60 & 1120 & 112 & 52.1 & 0.0835 & $\mathrm{v}$ & Dalk geen \\
\hline
\end{tabular}


Table 2 (continued).

\begin{tabular}{|c|c|c|c|c|c|c|c|c|c|c|c|c|c|c|c|c|c|c|}
\hline \multirow{2}{*}{$\begin{array}{l}\text { Core, section, } \\
\text { interval }(\mathrm{cm})\end{array}$} & \multirow[b]{2}{*}{$\begin{array}{l}\text { Depth } \\
\text { (mbsf) }\end{array}$} & \multicolumn{9}{|c|}{ Major elements (wt\%) } & \multicolumn{4}{|c|}{ Trace elements $(\mu \mathrm{g} / \mathrm{g})$} & \multirow[b]{2}{*}{$\begin{array}{c}\mathrm{CaCO}_{3} \\
(\mathrm{wt} \%)\end{array}$} & \multirow[b]{2}{*}{$\mathrm{Ti} / \mathrm{Al}$} & & \\
\hline & & $\mathrm{SiO}_{2}$ & $\mathrm{TiO}_{2}$ & $\mathrm{Al}_{2} \mathrm{O}_{3}$ & $\mathrm{Fe}_{2} \mathrm{O}_{3}$ & $\mathrm{MnO}$ & $\mathrm{MgO}$ & $\mathrm{CaO}$ & $\mathrm{Na}_{2} \mathrm{O}$ & $\mathrm{K}_{2} \mathrm{O}$ & $\mathrm{Ba}$ & $\mathrm{Cr}$ & $\mathrm{Sr}$ & $\mathrm{Zr}$ & & & Group & Color \\
\hline $15 \mathrm{H}-5,118-120$ & 139.59 & 24.3 & 0.675 & 8.74 & 3.33 & 0.0926 & 1.97 & 28.9 & 1.35 & 1.96 & 292 & 62 & 1080 & 116 & 51.6 & 0.0876 & $\mathrm{v}$ & Gray \\
\hline $15 \mathrm{H}-6,34-36$ & 140.25 & 24.5 & 0.454 & 9.11 & 3.27 & 0.0841 & 2.50 & 28.2 & 1.04 & 2.09 & 308 & 62 & 1030 & 75 & 50.3 & 0.0565 & o & \\
\hline $15 \mathrm{H}-6,67-69$ & 140.58 & 24.2 & 0.441 & 8.88 & 3.22 & 0.0908 & 2.45 & 28.5 & 1.05 & 2.20 & 304 & 70 & 1010 & 74 & 50.8 & 0.0562 & o & Gray/green \\
\hline $15 \mathrm{H}-6,116-118$ & 141.07 & 34.8 & 0.638 & 13.5 & 5.09 & 0.0390 & 2.12 & 16.1 & 1.30 & 1.92 & 482 & 132 & 670 & 119 & 28.8 & 0.0534 & o & \\
\hline $15 \mathrm{H}-\mathrm{CC}, 9-11$ & 141.23 & 34.8 & 0.640 & 13.5 & 6.11 & 0.0396 & 2.13 & 16.2 & 1.32 & 1.95 & 491 & 129 & 675 & 119 & 28.9 & 0.0537 & o & \\
\hline $16 \mathrm{H}-1,10-12$ & 142.01 & 35.4 & 0.632 & 13.0 & 4.90 & 0.0382 & 2.14 & 16.8 & 1.30 & 1.90 & 467 & 121 & 701 & 125 & 30.0 & 0.0551 & o & \\
\hline $16 \mathrm{H}-1,66-68$ & 142.55 & 34.7 & 0.604 & 12.3 & 5.97 & 0.0373 & 2.02 & 17.0 & 1.28 & 1.96 & 458 & 114 & 684 & 129 & 30.4 & 0.0556 & o & \\
\hline $16 \mathrm{H}-1,117-119$ & 143.04 & 34.4 & 0.600 & 12.2 & 4.51 & 0.0372 & 2.00 & 17.9 & 1.27 & 1.97 & 455 & 111 & 711 & 132 & 32.0 & 0.0557 & o & Dark green \\
\hline $16 \mathrm{H}-2,28-30$ & 143.62 & 27.9 & 0.528 & 10.3 & 3.76 & 0.0691 & 3.06 & 23.0 & 1.12 & 2.47 & 274 & 68 & 815 & 85 & 41.1 & 0.0583 & o & 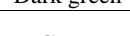 \\
\hline $16 \mathrm{H}-2,45-47$ & 143.79 & 28.1 & 0.532 & 10.4 & 3.86 & 0.0665 & 3.12 & 23.1 & 1.15 & 2.52 & 273 & 67 & 808 & 87 & 41.3 & 0.0582 & o & Green \\
\hline $16 \mathrm{H}-2,102-104$ & 144.32 & 28.3 & 0.510 & 10.3 & 3.96 & 0.106 & 3.12 & 24.4 & 1.05 & 2.44 & 263 & 73 & 831 & 83 & 43.6 & 0.0563 & o & Gray \\
\hline $16 \mathrm{H}-3,8-10$ & 144.88 & 28.6 & 0.530 & 10.9 & 3.74 & 0.0633 & 2.60 & 23.0 & 1.14 & 2.38 & 327 & 71 & 863 & 93 & 41.1 & 0.0551 & o & Gray \\
\hline $16 \mathrm{H}-3,68-70$ & 145.46 & $\begin{array}{l}28.0 \\
28.3\end{array}$ & 0.523 & 10.5 & $\begin{array}{l}5.14 \\
4.01\end{array}$ & 0.0639 & 2.53 & 22.7 & $\begin{array}{l}1.14 \\
1.10\end{array}$ & 2.35 & 308 & 74 & 830 & 94 & $\begin{array}{l}1.1 \\
40.5\end{array}$ & 0.0564 & 0 & \\
\hline $16 \mathrm{H}-3,117-119$ & 145.93 & 28.8 & 0.533 & 10.3 & 3.71 & 0.0676 & 2.50 & 24.2 & 1.14 & 2.28 & 315 & 64 & 901 & 97 & 43.2 & 0.0584 & o & Gray \\
\hline $16 \mathrm{H}-4,6-8$ & 146.31 & 46.3 & 0.988 & 21.4 & 6.68 & 0.0387 & 1.71 & 3.93 & 1.30 & 2.22 & 358 & 173 & 247 & 171 & 7.02 & 0.0522 & o & ( \\
\hline $16 \mathrm{H}-4,29-31$ & 146.53 & 44.6 & 0.956 & 20.8 & 7.07 & 0.0344 & 1.64 & 3.77 & 1.29 & 2.18 & 356 & 164 & 239 & 164 & 6.73 & 0.0520 & o & \\
\hline $16 \mathrm{H}-4,46-48$ & 146.69 & 46.1 & 0.992 & 21.5 & 6.64 & 0.0385 & 1.68 & 4.09 & 1.28 & 2.21 & 359 & 170 & 251 & 173 & 7.29 & 0.0524 & o & Dark green \\
\hline $16 \mathrm{H}-4,117-119$ & 147.38 & 5.24 & 0.126 & 1.87 & 0.824 & 0.0990 & 0.542 & 49.9 & 0.95 & 0.37 & 38 & 18 & 1460 & 34 & 89.1 & 0.0761 & $\mathrm{c}$ & 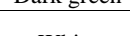 \\
\hline $16 \mathrm{H}-5,2-5$ & 147.72 & 2.04 & 0.0725 & 0.714 & 0.388 & 0.0635 & 0.464 & 51.0 & 1.11 & 0.14 & 16 & 3 & 1060 & 20 & 91.0 & 0.115 & c & White \\
\hline $16 \mathrm{H}-5,21-23$ & 147.90 & 30.3 & 0.577 & 11.8 & 4.46 & 0.0700 & 3.32 & 20.7 & 1.09 & 2.97 & 244 & 76 & 727 & 131 & 36.9 & 0.0555 & o & Gray \\
\hline $16 \mathrm{H}-5,87-89$ & 148.54 & 19.3 & 0.907 & 6.54 & 3.42 & 0.102 & 1.52 & 31.0 & 1.98 & 1.46 & 262 & 19 & 1170 & 194 & 55.3 & 0.157 & $\mathrm{v}$ & Uiay \\
\hline $16 \mathrm{H}-5,117-119$ & 148.83 & 19.7 & 0.914 & 6.79 & 3.33 & 0.113 & 1.58 & 32.8 & 2.05 & 1.50 & 261 & 20 & 1240 & 178 & 58.6 & 0.152 & $\mathrm{v}$ & Gray \\
\hline $16 \mathrm{H}-6,2-4$ & 149.17 & 30.8 & 0.564 & 11.8 & 3.88 & 0.0684 & 2.86 & 21.4 & 1.09 & 2.66 & 311 & 85 & 771 & 99 & 38.3 & 0.0544 & o & Gray \\
\hline $16 \mathrm{H}-6,60-62$ & 149.73 & 20.4 & 0.384 & 7.73 & 2.63 & 0.0690 & 1.74 & 32.6 & 1.06 & 1.72 & 311 & 54 & 1170 & 74 & 58.2 & 0.0562 & o & Gray \\
\hline $16 \mathrm{H}-6,70-72$ & 149.83 & 20.3 & 0.384 & 7.70 & 2.62 & 0.0744 & 1.77 & 32.2 & 1.03 & 1.60 & 301 & 46 & 1170 & 75 & 57.5 & 0.0565 & o & Gray \\
\hline $16 \mathrm{H}-6,136-138$ & 150.46 & 18.8 & 0.415 & 7.09 & 2.69 & 0.0858 & 1.90 & 33.3 & 1.09 & 1.58 & 260 & 51 & 1270 & 77 & 59.4 & 0.0663 & o & (3) \\
\hline $16 \mathrm{H}-7,71-73$ & 151.20 & 19.3 & 0.444 & 6.92 & 2.65 & 0.103 & 1.84 & 34.2 & 1.09 & 1.62 & 263 & 48 & 1300 & 84 & 61.0 & 0.0727 & o & \\
\hline $16 \mathrm{H}-\mathrm{CC}, 11-13$ & 151.33 & 18.6 & 0.433 & 6.71 & 2.60 & 0.104 & 1.81 & 33.3 & 1.09 & 1.45 & 249 & 52 & 1280 & 83 & 59.5 & 0.0731 & o & Gray/green \\
\hline $17 \mathrm{H}-1,48-50$ & 151.59 & 30.4 & 0.550 & 11.3 & 4.06 & 0.0554 & 2.93 & 21.2 & 1.16 & 2.51 & 315 & 84 & 772 & 100 & 37.8 & 0.0551 & o & Oray/gitem \\
\hline $17 \mathrm{H}-1,69-71$ & 151.78 & 30.7 & 0.564 & 11.3 & 4.11 & 0.0568 & 2.92 & 22.1 & 1.14 & 2.51 & 299 & 82 & 810 & 103 & 39.4 & 0.0564 & o & Green \\
\hline $17 \mathrm{H}-2,50-52$ & 152.06 & 23.0 & 0.426 & 8.63 & 3.09 & 0.0587 & 2.52 & 29.4 & 0.98 & 2.17 & 262 & 73 & 1010 & 70 & 52.4 & 0.0559 & o & \\
\hline $17 \mathrm{H}-2,63-65$ & 152.17 & 24.6 & 0.459 & 8.81 & 3.32 & 0.0638 & 2.74 & 29.4 & 1.00 & 2.12 & 253 & 68 & 1020 & 80 & 52.4 & 0.0590 & o & Green \\
\hline $17 \mathrm{H}-3,26-28$ & 152.65 & 2.97 & 0.0721 & 1.10 & 0.570 & 0.115 & 0.367 & 50.8 & 0.79 & 0.21 & 32 & 20 & 1570 & 20 & 90.7 & 0.0744 & $\mathrm{c}$ & \\
\hline $17 \mathrm{H}-3,35-37$ & 152.73 & 3.54 & 0.0826 & 1.20 & 0.699 & 0.108 & 0.421 & 51.1 & 0.88 & 0.23 & 33 & 15 & 1610 & 24 & 91.2 & 0.0783 & $\mathrm{c}$ & White \\
\hline $17 \mathrm{H}-3,122-123$ & 153.43 & 41.5 & 0.879 & 18.3 & 5.60 & 0.023 & 1.70 & 9.06 & 1.17 & 1.98 & 362 & 137 & 429 & 148 & 16.2 & 0.0544 & o & wime \\
\hline $17 \mathrm{H}-3,130-131$ & 153.50 & 40.8 & 0.863 & 18.0 & 5.57 & 0.0233 & 1.67 & 8.97 & 1.17 & 1.93 & 355 & 135 & 425 & 145 & 16.0 & 0.0543 & o & \\
\hline $17 \mathrm{H}-3,138-139$ & 153.57 & $\begin{array}{l}40.0 \\
41.4\end{array}$ & 0.873 & $\begin{array}{l}18.0 \\
18.2\end{array}$ & 5.69 & 0.0236 & 1.70 & 9.12 & 1.35 & 2.00 & 373 & 137 & 432 & 154 & $\begin{array}{l}10.0 \\
16.3\end{array}$ & 0.0542 & o & \\
\hline $17 \mathrm{H}-4,14-16$ & 153.78 & 44.1 & 0.931 & 19.2 & 6.35 & 0.0256 & 1.79 & 8.27 & 1.33 & 2.11 & 378 & 168 & 415 & 165 & 14.8 & 0.0549 & o & Dark green \\
\hline $17 \mathrm{H}-4,60-62$ & 154.15 & $\begin{array}{l}4.1 \\
27.4\end{array}$ & $\begin{array}{l}0.931 \\
0.497\end{array}$ & $\begin{array}{l}19.2 \\
10.1\end{array}$ & $\begin{array}{l}0.35 \\
3.55\end{array}$ & $\begin{array}{l}0.0250 \\
0.0542\end{array}$ & 2.72 & 25.0 & $\begin{array}{l}1.33 \\
1.08\end{array}$ & $\begin{array}{l}2.11 \\
2.22\end{array}$ & 290 & $\begin{array}{r}108 \\
78\end{array}$ & 916 & $\begin{array}{r}103 \\
93\end{array}$ & $\begin{array}{l}44.8 \\
44.7\end{array}$ & $\begin{array}{l}0.0349 \\
0.0557\end{array}$ & $\begin{array}{l}0 \\
0\end{array}$ & Dark green \\
\hline $17 \mathrm{H}-\mathrm{CC}, 22-24$ & 154.35 & 27.6 & 0.492 & 9.51 & 3.40 & 0.0559 & 2.72 & 24.3 & 1.06 & 2.13 & 287 & 83 & 857 & 97 & 43.4 & 0.0586 & o & Green \\
\hline $18 \mathrm{X}-1,111-113$ & 154.65 & $\begin{array}{l}28.0 \\
28.1\end{array}$ & 0.503 & 9.59 & 3.97 & 0.0574 & 2.78 & 24.8 & 1.07 & 2.31 & 317 & $\begin{array}{l}53 \\
70\end{array}$ & 857 & $\begin{array}{l}91 \\
99\end{array}$ & $\begin{array}{l}45.4 \\
44.3\end{array}$ & 0.0594 & o & Oreent \\
\hline $18 \mathrm{X}-1,128-130$ & 154.82 & 28.1 & 0.501 & 9.42 & 3.52 & 0.0585 & 2.79 & 25.1 & 1.16 & 2.19 & 297 & 65 & 861 & 101 & 44.8 & 0.0602 & o & Pale green \\
\hline $18 \mathrm{X}-1,143-145$ & 154.97 & 15.4 & 0.283 & 5.83 & 1.97 & 0.0832 & 1.35 & 38.4 & 0.96 & 1.11 & 335 & 52 & 1360 & 58 & 68.6 & 0.0549 & o & Gray \\
\hline $18 \mathrm{X}-2,47-49$ & 155.50 & 28.1 & 0.506 & 10.3 & 3.68 & 0.0589 & 2.86 & 24.0 & 1.07 & 2.50 & 306 & 73 & 838 & 95 & 42.8 & 0.0555 & o & Giay \\
\hline $18 X-2,94-96$ & 155.97 & 27.6 & 0.496 & 9.88 & 4.64 & 0.0622 & 2.79 & 23.6 & 1.07 & 2.36 & 290 & 68 & 818 & 95 & 42.1 & 0.0569 & o & \\
\hline $18 \mathrm{X}-2,118-120$ & 156.21 & 28.0 & 0.503 & 9.89 & 3.49 & 0.0632 & 2.77 & 24.9 & 1.06 & 2.33 & 300 & 68 & 860 & 98 & 44.4 & 0.0576 & o & Green \\
\hline $18 \mathrm{X}-\mathrm{CC}, 10-12$ & 157.01 & 28.0 & 0.520 & 10.3 & 3.52 & 0.0473 & 2.60 & 24.4 & 1.09 & 2.27 & 300 & 75 & 858 & 98 & 43.5 & 0.0571 & o & Givenin \\
\hline $18 \mathrm{X}-\mathrm{CC}, 37-39$ & 157.28 & 27.4 & 0.508 & 9.98 & 3.52 & 0.0486 & 2.57 & 24.4 & 1.06 & 2.30 & 288 & 68 & 849 & 96 & 43.5 & 0.0577 & o & Green \\
\hline $19 \mathrm{X}-1,70-72$ & 160.84 & 13.8 & 0.258 & 5.09 & 1.74 & 0.0585 & 1.20 & 40.0 & 0.93 & 0.99 & 245 & 40 & 1410 & 54 & 71.4 & 0.0573 & o & \\
\hline $19 X-1,87-89$ & 161.01 & 15.3 & 0.279 & 4.99 & 1.76 & 0.0620 & 1.29 & 39.3 & 1.04 & 0.99 & 236 & 44 & 1320 & 68 & 70.1 & 0.0635 & o & Pale green \\
\hline $19 \mathrm{X}-1,135-137$ & 161.49 & 8.69 & $\begin{array}{l}0.321 \\
0.321\end{array}$ & 3.15 & $\begin{array}{l}1.70 \\
1.39\end{array}$ & $\begin{array}{l}0.0020 \\
0.0822\end{array}$ & $\begin{array}{l}1.29 \\
0.90\end{array}$ & 45.1 & 1.15 & 0.68 & $\begin{array}{l}230 \\
136\end{array}$ & $\begin{array}{l}44 \\
27\end{array}$ & $\begin{array}{l}1320 \\
1560\end{array}$ & $\begin{array}{l}08 \\
59\end{array}$ & 80.5 & $\begin{array}{l}0.0035 \\
0.115\end{array}$ & $\begin{array}{l}0 \\
\mathrm{c}\end{array}$ & Pale green \\
\hline $19 \mathrm{X}-2,15-17$ & 161.72 & 8.79 & 0.330 & 3.23 & 1.31 & 0.0849 & 0.89 & 44.7 & 1.26 & 0.71 & 140 & 22 & 1560 & 55 & 79.8 & 0.116 & c & \\
\hline $19 \mathrm{X}-2,40-42$ & 161.97 & 9.43 & 0.362 & 3.45 & 1.36 & 0.0838 & 0.94 & 44.1 & 1.40 & 0.74 & 142 & 24 & 1550 & 55 & 78.8 & 0.119 & c & Gray \\
\hline $19 X-2,66-68$ & 162.23 & 27.4 & 0.503 & 10.3 & 3.73 & 0.0799 & 3.58 & 24.2 & 1.02 & 2.58 & 246 & 75 & 805 & 88 & 43.2 & 0.0554 & o & Gray \\
\hline $19 \mathrm{X}-2,103-105$ & 162.60 & 3.13 & 0.0875 & 1.07 & 0.491 & 0.0754 & 0.394 & 51.2 & 0.80 & 0.26 & 46 & 9 & 1410 & 22 & 91.4 & 0.0923 & $\mathrm{c}$ & White \\
\hline $19 \mathrm{X}-2,141-143$ & 162.98 & 3.02 & 0.0647 & 1.06 & 0.460 & 0.0767 & 0.384 & 51.7 & 0.80 & 0.25 & 43 & 8 & 1560 & 21 & 92.2 & 0.0689 & $\mathrm{c}$ & White \\
\hline $19 \mathrm{X}-3,38-40$ & 163.45 & 3.44 & 0.0855 & 1.18 & 0.511 & 0.0685 & 0.412 & 50.9 & 0.80 & 0.27 & 46 & 7 & 1510 & 20 & 90.8 & 0.0823 & $\mathrm{c}$ & $\begin{array}{l}\text { White } \\
\text { White }\end{array}$ \\
\hline $19 \mathrm{X}-3,118-120$ & 164.25 & 23.8 & 0.437 & 8.63 & 3.08 & 0.0551 & 2.66 & 28.7 & 1.08 & 1.91 & 268 & 57 & 1010 & 84 & 51.3 & 0.0574 & o & wnite \\
\hline $19 \mathrm{X}-4,39-41$ & 164.96 & 24.3 & 0.440 & 8.44 & 3.06 & 0.0591 & 2.76 & 27.9 & 1.07 & 1.88 & 261 & 53 & 964 & 87 & 49.8 & 0.0591 & o & \\
\hline $19 \mathrm{X}-4,127-129$ & 165.84 & 26.7 & 0.461 & 7.60 & 2.81 & 0.0732 & 2.80 & 28.2 & 1.27 & 1.71 & 253 & 49 & 919 & 106 & 50.4 & 0.0688 & o & Green \\
\hline $19 \mathrm{X}-5,27-29$ & $\begin{array}{l}10.04 \\
166.34\end{array}$ & 31.4 & 0.564 & 11.1 & 4.78 & 0.0274 & 2.14 & $\begin{array}{l}2.2 \\
19.7\end{array}$ & 1.23 & 1.71 & 360 & 101 & 769 & 119 & 35.2 & 0.0575 & o & Uicent \\
\hline $19 \mathrm{X}-5,61-63$ & 166.68 & 33.2 & $\begin{array}{l}0.504 \\
0.597\end{array}$ & 11.6 & 4.36 & $\begin{array}{l}0.0296 \\
0.0296\end{array}$ & 2.23 & 20.8 & 1.25 & 1.77 & 370 & $\begin{array}{l}104 \\
104\end{array}$ & 815 & 126 & 37.1 & 0.0582 & o & \\
\hline $19 X-5,93-95$ & 167.00 & 32.8 & 0.590 & 11.6 & 3.89 & 0.0293 & 2.21 & 20.1 & 1.21 & 1.74 & 366 & 103 & 777 & 121 & 35.9 & 0.0577 & o & Dark green \\
\hline $19 \mathrm{X}-5,144-146$ & 167.51 & 20.2 & 0.394 & 7.16 & 2.86 & 0.0852 & 2.83 & 33.2 & 0.96 & $\begin{array}{l}1.69 \\
\end{array}$ & 252 & 50 & 1110 & 65 & 59.2 & 0.0624 & o & Daingetit \\
\hline $19 \mathrm{X}-6,28-30$ & 167.85 & 20.4 & 0.399 & 7.11 & 2.86 & 0.0844 & 2.78 & 32.2 & 0.97 & 1.70 & 239 & 49 & 1080 & 67 & 57.5 & 0.0636 & o & \\
\hline
\end{tabular}


Table 2 (continued).

\begin{tabular}{|c|c|c|c|c|c|c|c|c|c|c|c|c|c|c|c|c|c|c|}
\hline \multirow[b]{2}{*}{$\begin{array}{l}\text { Core, section, } \\
\text { interval }(\mathrm{cm})\end{array}$} & \multirow[b]{2}{*}{$\begin{array}{l}\text { Depth } \\
\text { (mbsf) }\end{array}$} & \multicolumn{9}{|c|}{ Major elements (wt\%) } & \multicolumn{4}{|c|}{ Trace elements $(\mu \mathrm{g} / \mathrm{g})$} & \multirow[b]{2}{*}{$\begin{array}{l}\mathrm{CaCO}_{3} \\
(\mathrm{wt} \%)\end{array}$} & & & \\
\hline & & $\mathrm{SiO}_{2}$ & $\mathrm{TiO}_{2}$ & $\mathrm{Al}_{2} \mathrm{O}_{3}$ & $\mathrm{Fe}_{2} \mathrm{O}_{3}$ & $\mathrm{MnO}$ & $\mathrm{MgO}$ & $\mathrm{CaO}$ & $\mathrm{Na}_{2} \mathrm{O}$ & $\mathrm{K}_{2} \mathrm{O}$ & $\mathrm{Ba}$ & $\mathrm{Cr}$ & $\mathrm{Sr}$ & $\mathrm{Zr}$ & & $\mathrm{Ti} / \mathrm{Al}$ & Group & Color \\
\hline $19 \mathrm{X}-6,62-64$ & 168.19 & 22.6 & 0.431 & 7.13 & 2.90 & 0.0870 & 2.87 & 30.8 & 1.06 & 1.71 & 245 & 48 & 1010 & 75 & 55.0 & 0.0685 & o & Gray/green \\
\hline 19X-CC, $9-11$ & 168.47 & 23.1 & 0.427 & 8.60 & 2.93 & 0.0682 & 2.58 & 29.0 & 1.01 & 1.91 & 275 & 60 & 1030 & 81 & 51.7 & 0.0562 & o & \\
\hline 19X-CC, $43-45$ & 168.81 & 22.3 & 0.412 & 8.31 & 5.05 & 0.0704 & 2.42 & 27.9 & 1.02 & 1.85 & 258 & 57 & 995 & 80 & 49.9 & 0.0562 & o & \\
\hline $20 \mathrm{X}-1,20-22$ & 169.97 & 23.8 & 0.444 & 8.38 & 3.60 & 0.0743 & 2.49 & 28.6 & 1.13 & 1.92 & 261 & 56 & 1010 & 86 & 51.0 & 0.0600 & o & \\
\hline $20 \mathrm{X}-1,61-63$ & 170.34 & 23.8 & 0.448 & 8.47 & 2.92 & 0.0714 & 2.37 & 29.1 & 1.08 & 1.91 & 252 & 57 & 1030 & 91 & 52.0 & 0.0598 & o & Green \\
\hline $20 \mathrm{X}-1,93-95$ & 170.62 & 15.8 & 0.496 & 5.55 & 2.28 & 0.130 & 1.21 & 38.5 & 1.27 & 1.16 & 184 & 29 & 1350 & 108 & 68.8 & 0.101 & $\mathrm{v}$ & (1) \\
\hline $20 \mathrm{X}-1,126-128$ & 170.91 & 13.5 & 0.781 & 4.78 & 2.24 & 0.108 & 1.24 & 39.2 & 1.67 & 0.99 & 206 & 25 & 1360 & 99 & 70.0 & 0.185 & $\mathrm{v}$ & Gray \\
\hline 20X-2, 97-99 & 171.96 & 26.3 & 0.481 & $\begin{array}{l}4.60 \\
9.61\end{array}$ & 3.20 & 0.0533 & $\begin{array}{l}1.24 \\
2.22\end{array}$ & 27.3 & $\begin{array}{l}1.01 \\
1.15\end{array}$ & 2.03 & 265 & 62 & 988 & 92 & 48.8 & 0.0568 & o & \\
\hline $20 \mathrm{X}-2,115-117$ & 172.12 & 26.3 & 0.490 & 10.0 & 3.34 & 0.0622 & 2.28 & 29.2 & 1.10 & 2.12 & 259 & 68 & 1060 & 108 & 52.2 & 0.0553 & o & Green \\
\hline $20 \mathrm{X}-3,5-7$ & 172.48 & $\begin{array}{l}20.3 \\
23.2\end{array}$ & $\begin{array}{l}0.490 \\
1.31\end{array}$ & 7.98 & $\begin{array}{l}5.34 \\
4.91\end{array}$ & $\begin{array}{l}0.0022 \\
0.115\end{array}$ & 2.25 & 28.2 & $\begin{array}{l}1.10 \\
2.38\end{array}$ & $\begin{array}{l}2.22 \\
1.26\end{array}$ & 204 & $\begin{array}{l}08 \\
44\end{array}$ & 1070 & $\begin{array}{l}108 \\
167\end{array}$ & 50.4 & 0.185 & $\begin{array}{l}0 \\
\mathrm{v}\end{array}$ & Green \\
\hline $\begin{array}{l}20 \times-3,5-1 \\
20 X-3,23-25\end{array}$ & $\begin{array}{l}1 / 2.48 \\
172.64\end{array}$ & 23.3 & $\begin{array}{l}1.31 \\
1.32\end{array}$ & $\begin{array}{l}.1 .98 \\
8.02\end{array}$ & $\begin{array}{l}4.91 \\
4.85\end{array}$ & $\begin{array}{l}0.115 \\
0.115\end{array}$ & 2.25 & $\begin{array}{l}28.2 \\
28.2\end{array}$ & $\begin{array}{l}2.38 \\
2.40\end{array}$ & $\begin{array}{l}1.26 \\
1.30\end{array}$ & $\begin{array}{l}204 \\
206\end{array}$ & $\begin{array}{l}44 \\
42\end{array}$ & $\begin{array}{l}10 / 0 \\
1070\end{array}$ & $\begin{array}{l}16 / \\
170\end{array}$ & $\begin{array}{l}50.4 \\
50.4\end{array}$ & $\begin{array}{l}0.185 \\
0.186\end{array}$ & $\begin{array}{llll}\mathrm{v} \\
\mathrm{v}\end{array}$ & \\
\hline $20 \mathrm{X}-3,46-48$ & 172.84 & 24.2 & 1.37 & 8.43 & 4.83 & 0.0978 & 2.27 & 26.6 & 2.48 & 1.39 & 225 & 39 & 1030 & 168 & 47.4 & 0.184 & $\mathrm{v}$ & Dark gray \\
\hline $20 X-3,106-108$ & 173.38 & 3.16 & 0.088 & 1.03 & 0.470 & 0.0860 & 0.600 & 50.8 & 1.03 & 0.24 & 41 & 10 & 1100 & 23 & 90.7 & 0.0966 & $\mathrm{c}$ & Pale gray \\
\hline $20 \mathrm{X}-4,41-43$ & 174.13 & 8.94 & 0.288 & 3.11 & 1.40 & 0.101 & 0.910 & 44.6 & 1.21 & 0.67 & 162 & 24 & 1570 & 54 & 79.6 & 0.105 & $\mathrm{c}$ & 1 aiv gita \\
\hline $20 \mathrm{X}-4,64-66$ & 174.33 & 8.81 & 0.290 & 3.05 & 1.40 & 0.109 & 0.870 & 45.1 & 1.19 & 0.67 & 161 & 28 & 1600 & 52 & 80.5 & 0.108 & $\mathrm{c}$ & Gray \\
\hline $20 \mathrm{X}-4,109-111$ & 174.73 & 17.9 & 0.309 & 6.43 & 2.16 & 0.0660 & 1.25 & 35.6 & 1.14 & 1.06 & 356 & 50 & 1290 & 61 & 63.6 & 0.0544 & o & Gray \\
\hline $20 \mathrm{X}-5,8-10$ & 175.17 & 38.4 & 0.763 & 16.1 & 5.40 & 0.0277 & 1.82 & 11.2 & 1.35 & 1.86 & 530 & 129 & 502 & 137 & 20.0 & 0.0538 & o & \\
\hline $20 X-5,49-51$ & 175.52 & 39.3 & 0.774 & 16.2 & 5.38 & 0.0281 & 1.85 & 11.5 & 1.29 & 1.90 & 543 & 130 & 510 & 140 & 20.5 & 0.0542 & o & \\
\hline $20 \mathrm{X}-5,110-112$ & 176.07 & 40.0 & 0.793 & 16.6 & 5.30 & 0.0287 & 1.84 & 11.0 & 1.33 & 1.90 & 506 & 134 & 496 & 144 & 19.7 & 0.0543 & o & Dark green \\
\hline $20 \mathrm{X}-6,39-41$ & 176.77 & 21.9 & 0.409 & 8.10 & 2.92 & 0.0703 & 2.13 & 31.4 & 1.06 & 1.77 & 285 & 60 & 1110 & 80 & 56.0 & 0.0571 & o & \\
\hline $20 \mathrm{X}-6,112-114$ & 177.42 & 21.9 & 0.406 & 8.09 & 2.86 & 0.0741 & 2.17 & 31.0 & 1.07 & 1.73 & 276 & 61 & 1100 & 78 & 55.4 & 0.0568 & o & \\
\hline 20X-CC, $21-23$ & 178.24 & 23.9 & 0.436 & 7.79 & 2.83 & 0.0710 & 2.20 & 29.8 & 1.09 & 1.71 & 265 & 55 & 1030 & 91 & 53.1 & 0.0634 & o & Green \\
\hline $21 \mathrm{X}-1,81-83$ & 179.27 & 15.5 & 0.283 & 5.60 & 1.87 & 0.0613 & 1.28 & 39.2 & 1.04 & 0.97 & 300 & 52 & 1380 & 61 & 70.0 & 0.0572 & o & \\
\hline $21 \mathrm{X}-1,109-110$ & 179.55 & 15.4 & 0.280 & 5.53 & 1.84 & 0.0628 & 1.29 & 38.1 & 1.10 & 1.02 & 284 & 53 & 1330 & 62 & 68.1 & 0.0574 & o & Pale green \\
\hline $21 \mathrm{X}-2,39-41$ & 180.33 & 23.9 & 0.439 & 8.45 & 3.05 & 0.0778 & 2.37 & 29.4 & 1.03 & 1.76 & 319 & 70 & 1020 & 85 & 52.4 & 0.0589 & 0 & 1 aic gictil \\
\hline $21 \mathrm{X}-2,107-109$ & 181.00 & 24.1 & 0.444 & $\begin{array}{l}0.43 \\
8.38\end{array}$ & 3.29 & 0.0853 & 2.37 & 29.0 & 1.06 & 1.84 & 309 & 65 & 1010 & 86 & 51.8 & 0.0601 & 0 & \\
\hline $21 \mathrm{X}-3,40-42$ & 181.84 & 24.8 & 0.446 & 8.16 & 2.95 & 0.0875 & 2.28 & 29.2 & 1.11 & 1.74 & 315 & 66 & 1000 & 91 & 52.1 & 0.0619 & o & Green \\
\hline $21 X-3,124-126$ & 182.67 & 32.5 & 0.564 & 11.6 & 3.99 & 0.0374 & 1.98 & 18.9 & 1.16 & 1.73 & 470 & 107 & 709 & 124 & 33.7 & 0.0552 & o & \\
\hline $21 X-4,6-8$ & 182.99 & 33.1 & 0.575 & 11.6 & 4.18 & 0.0410 & 2.00 & 19.4 & 1.20 & 1.66 & 460 & 108 & 726 & 124 & 34.5 & 0.0562 & o & \\
\hline $21 \mathrm{X}-4,62-64$ & 183.55 & 34.6 & 0.595 & 12.1 & 4.05 & 0.0446 & 2.06 & 20.3 & 1.26 & 1.76 & 467 & 109 & 762 & 129 & 36.3 & 0.0556 & o & Dark green \\
\hline $21 \mathrm{X}-4,119-121$ & 184.11 & 28.4 & 0.504 & 10.5 & 3.53 & 0.0622 & 2.38 & 25.2 & 1.12 & 2.27 & 342 & 77 & 900 & 90 & 44.9 & 0.0543 & o & \\
\hline $21 \mathrm{X}-4,145-147$ & 184.37 & 30.5 & 0.536 & 10.9 & 3.89 & 0.0656 & 2.49 & 26.2 & 1.18 & 2.43 & 355 & 79 & 925 & 98 & 46.8 & 0.0556 & o & Green \\
\hline $21 X-5,19-21$ & 184.61 & 26.6 & 0.516 & 9.79 & 3.30 & 0.0696 & 2.92 & 26.6 & 1.16 & 2.38 & 275 & 76 & 888 & 87 & 47.5 & 0.0597 & o & \\
\hline $21 \mathrm{X}-5,88-90$ & $\begin{array}{l}184.01 \\
185.29\end{array}$ & $\begin{array}{l}20.0 \\
17.1\end{array}$ & $\begin{array}{l}0.010 \\
0.284\end{array}$ & 5.95 & 2.26 & $\begin{array}{l}0.0090 \\
0.0791\end{array}$ & 1.24 & $\begin{array}{l}20.0 \\
38.1\end{array}$ & $\begin{array}{l}1.10 \\
0.90\end{array}$ & $\begin{array}{l}2.38 \\
1.10\end{array}$ & 323 & $\begin{array}{l}10 \\
55\end{array}$ & $\begin{array}{r}808 \\
1310\end{array}$ & $\begin{array}{l}81 \\
62\end{array}$ & 68.1 & 0.0541 & $\begin{array}{l}0 \\
0\end{array}$ & Green \\
\hline $21 X-5,103-105$ & 185.44 & 19.3 & 0.328 & 6.75 & 2.55 & 0.0647 & 1.37 & 36.8 & 1.01 & 1.21 & 357 & 59 & 1310 & 71 & 65.6 & 0.0551 & o & Pale green \\
\hline $21 X-5,125-127$ & 185.66 & 5.31 & 0.125 & 1.84 & 0.766 & 0.134 & 0.533 & 50.2 & 1.09 & 0.39 & 60 & 26 & 1500 & 34 & 89.5 & 0.0770 & $\mathrm{c}$ & \\
\hline $21 \mathrm{X}-6,4-6$ & 185.95 & 3.74 & 0.098 & 1.29 & 0.573 & 0.139 & 0.437 & 52.1 & 0.98 & 0.27 & 53 & 25 & 1520 & 29 & 93.0 & 0.0859 & $\mathrm{c}$ & White \\
\hline 21X-6, 89-91 & 186.79 & 13.8 & 0.277 & 5.02 & 1.84 & 0.0726 & 1.12 & 40.6 & 1.12 & 0.98 & 243 & 48 & 1430 & 64 & 72.4 & 0.0625 & o & wimle \\
\hline $21 X-6,142-144$ & 187.32 & 14.1 & 0.281 & 5.07 & 1.84 & 0.0835 & 1.12 & 41.3 & 1.05 & 1.01 & 243 & 49 & 1440 & $\begin{array}{l}04 \\
66\end{array}$ & 73.7 & 0.0629 & 0 & \\
\hline $21 \mathrm{X}-\mathrm{CC}, 7-9$ & 187.97 & 15.0 & 0.300 & 5.11 & 1.79 & 0.0856 & 1.11 & 40.2 & 1.03 & 1.00 & 245 & 46 & 1410 & 70 & 71.7 & 0.0664 & o & Gray \\
\hline $22 \mathrm{X}-1,62-64$ & 188.81 & 8.91 & 0.203 & 3.05 & 1.26 & 0.141 & 0.738 & 46.0 & 0.92 & 0.60 & 53 & 31 & 1360 & 46 & 82.0 & 0.0755 & c & \\
\hline $22 \mathrm{X}-1,84-86$ & 189.02 & 8.58 & 0.217 & 2.88 & 1.18 & 0.143 & 0.788 & 46.4 & 1.07 & 0.58 & 56 & 31 & 1270 & 47 & 82.8 & 0.0850 & $\mathrm{c}$ & White \\
\hline $22 \mathrm{X}-2,3-5$ & 189.69 & 26.9 & 0.481 & 9.62 & 3.30 & 0.0466 & $\begin{array}{l}0.680 \\
1.68\end{array}$ & 27.6 & 1.09 & 1.37 & 372 & 89 & 1010 & 104 & $\begin{array}{l}49.3 \\
-19.3\end{array}$ & 0.0567 & o & \\
\hline $22 \mathrm{X}-2,48-50$ & 190.12 & 25.8 & 0.457 & 9.16 & 3.28 & 0.0497 & 1.64 & 27.2 & 1.08 & 1.34 & 357 & 84 & 992 & $\begin{array}{r}104 \\
99\end{array}$ & 48.6 & 0.0565 & 0 & \\
\hline $22 \mathrm{X}-2,87-89$ & 190.50 & 26.4 & 0.469 & 9.40 & 3.07 & 0.0542 & 1.66 & 28.7 & 1.14 & 1.37 & 363 & 91 & 1050 & 102 & 51.2 & 0.0566 & o & Dark green \\
\hline $22 \mathrm{X}-3,26-28$ & 191.37 & 20.5 & $\begin{array}{l}0.409 \\
0.401\end{array}$ & $\begin{array}{l}.4 .40 \\
7.72\end{array}$ & 2.72 & $\begin{array}{l}0.0342 \\
0.0728\end{array}$ & $\begin{array}{l}1.00 \\
1.89\end{array}$ & 34.4 & $\begin{array}{l}1.14 \\
1.10\end{array}$ & $\begin{array}{l}1.51 \\
1.58\end{array}$ & $\begin{array}{l}303 \\
244\end{array}$ & $\begin{array}{l}91 \\
57\end{array}$ & 1230 & $\begin{array}{r}102 \\
79\end{array}$ & 61.5 & $\begin{array}{l}0.0300 \\
0.0588\end{array}$ & o & Dark green \\
\hline $\begin{array}{l}22 \mathrm{X}-3,2 \mathrm{C}-28 \\
22 \mathrm{X}-3,98-100\end{array}$ & 192.07 & $\begin{array}{l}20.9 \\
19.9\end{array}$ & $\begin{array}{l}0.401 \\
0.385\end{array}$ & 7.47 & 2.65 & $\begin{array}{l}0.0728 \\
0.0692\end{array}$ & $\begin{array}{l}1.89 \\
1.86\end{array}$ & $\begin{array}{l}34.4 \\
33.4\end{array}$ & $\begin{array}{l}1.10 \\
0.99\end{array}$ & $\begin{array}{l}1.58 \\
1.60\end{array}$ & 243 & 57 & $\begin{array}{l}1230 \\
1180\end{array}$ & 77 & $\begin{array}{l}0.3 \\
59.6\end{array}$ & $\begin{array}{l}0.0388 \\
0.0584\end{array}$ & $\begin{array}{l}0 \\
0\end{array}$ & \\
\hline $22 \mathrm{X}-4,37-39$ & 192.93 & 23.7 & 0.433 & 8.60 & 3.06 & 0.0785 & 2.10 & 30. & 1.00 & 1.83 & 253 & 65 & 1090 & 82 & 54.7 & 0.0570 & o & Gray \\
\hline $22 \mathrm{X}-5,58-60$ & 194.59 & 20.7 & 0.401 & 7.57 & 2.73 & 0.0684 & 1.94 & 33. & 1.06 & 1.60 & 234 & 58 & 1180 & 81 & 60.0 & 0.0601 & 0 & \\
\hline $22 \mathrm{X}-6,92-94$ & 196.39 & 20.7 & 0.402 & 7.53 & 2.73 & 0.0703 & 1.92 & 33. & 1.02 & 1.60 & 245 & 58 & 118 & $\begin{array}{l}01 \\
82\end{array}$ & 59.5 & 0.0605 & o & \\
\hline $22 \mathrm{X}-\mathrm{CC}, 24-26$ & 197.66 & 23.8 & 0.465 & 7.63 & 2.92 & 0.0820 & 1.99 & 32.1 & 1.06 & 1.66 & 259 & $\begin{array}{l}50 \\
59\end{array}$ & 1110 & 98 & 57.3 & 0.0691 & o & \\
\hline $23 \mathrm{X}-1,14-16$ & 197.95 & 25.6 & 0.501 & 7.67 & 2.96 & 0.0847 & 2.0 & 30.6 & 1.08 & 1.65 & 267 & 58 & 1060 & 110 & 54.6 & 0.0740 & o & \\
\hline $23 \mathrm{X}-1,36-38$ & 198.17 & 27.8 & 0.530 & 7.52 & 2.93 & 0.0819 & 2.00 & 28.7 & 1.15 & 1.59 & 273 & 55 & 978 & 127 & 51.2 & 0.0799 & o & Gray \\
\hline $23 \mathrm{X}-2,8-10$ & 199.35 & 4.23 & 0.119 & 1.46 & 0.671 & 0.0919 & 0.451 & 50.7 & 0.99 & 0.34 & 37 & 27 & 1430 & 33 & 90.5 & 0.0925 & $\mathrm{c}$ & \\
\hline $23 \mathrm{X}-2,55-57$ & 199.82 & 4.53 & 0.158 & 1.51 & 0.742 & 0.0785 & 0.511 & 50.4 & 0.98 & 0.34 & 49 & 29 & 1260 & 37 & 90.0 & 0.119 & $\mathrm{c}$ & White \\
\hline $23 \mathrm{X}-2,142-144$ & 200.69 & 13.9 & 0.375 & 4.92 & 2.19 & 0.0833 & 1.21 & 41.0 & 0.93 & 1.03 & 173 & 42 & 1400 & 76 & 73.2 & 0.0864 & $\mathrm{v}$ & \\
\hline $23 \mathrm{X}-3,26-28$ & 201.03 & 12.9 & 0.381 & 4.68 & 1.90 & 0.0893 & 1.14 & 40.8 & 1.12 & 0.97 & 178 & 43 & 1380 & 78 & 72.7 & 0.0924 & $\mathrm{v}$ & Gray \\
\hline $23 \mathrm{X}-3,119-121$ & 201.96 & 26.3 & $\begin{array}{l}0.301 \\
0.485\end{array}$ & $\begin{array}{l}4.00 \\
9.95\end{array}$ & 3.12 & 0.0481 & $\begin{array}{l}1.14 \\
1.64\end{array}$ & $\begin{array}{l}40.0 \\
27.7\end{array}$ & 1.06 & 1.44 & 311 & 82 & 1030 & 93 & 49.4 & 0.0553 & o & Oray \\
\hline $23 \mathrm{X}-4,35-37$ & 202.62 & $\begin{array}{l}28.3 \\
28.0\end{array}$ & $\begin{array}{l}0.485 \\
0.508\end{array}$ & $\begin{array}{l}9.93 \\
10.1\end{array}$ & 3.42 & $\begin{array}{l}0.0481 \\
0.0544\end{array}$ & $\begin{array}{l}1.04 \\
1.69\end{array}$ & 28.5 & $\begin{array}{l}1.00 \\
1.10\end{array}$ & $\begin{array}{l}1.44 \\
1.57\end{array}$ & 331 & $\begin{array}{l}82 \\
84\end{array}$ & $\begin{array}{l}1030 \\
1050\end{array}$ & $\begin{array}{r}93 \\
102\end{array}$ & $\begin{array}{l}49.4 \\
50.9\end{array}$ & $\begin{array}{l}0.0533 \\
0.0568\end{array}$ & $\begin{array}{l}0 \\
0 \\
0\end{array}$ & \\
\hline $23 \mathrm{X}-4,114-116$ & 203.41 & 28.4 & 0.495 & 9.57 & 3.25 & 0.0515 & 1.61 & 26.1 & 1.04 & 1.52 & 325 & 80 & 945 & 109 & 46.5 & 0.0586 & o & Green \\
\hline $23 X-5,71-73$ & 204.48 & 4.29 & 0.0992 & 1.53 & 0.654 & 0.126 & 0.470 & 49.9 & 0.93 & 0.33 & 34 & 26 & 1480 & 32 & 89.1 & 0.0733 & $\mathrm{c}$ & \\
\hline 0507 & 204.72 & 3.53 & 0.0846 & 1.24 & 0.566 & 0.135 & 0.422 & 50 & 0.83 & 0.2 & 28 & 23 & 1480 & 27 & $\begin{array}{l}8.1 \\
90.9\end{array}$ & 0.0770 & $\mathrm{c}$ & White \\
\hline $144-146$ & 205.21 & 15.2 & $\begin{array}{l}0.0040 \\
0.294\end{array}$ & $\begin{array}{l}1.24 \\
5.80\end{array}$ & 1. & 3 & 1. & 38 & 0.8 & 1.8 r & 239 & 50 & 138 & 59 & 68 & 0 & o & Gray \\
\hline $23 \mathrm{X}-6,28-30$ & 20 & 25.8 & 0.471 & $\begin{array}{l}9.80 \\
9.89 \\
\end{array}$ & $\begin{array}{l}1.9 \\
3.2\end{array}$ & 0.0 & 2. & 26 & $\begin{array}{l}0.92 \\
0.99\end{array}$ & 2.1. & 249 & $\begin{array}{l}50 \\
69\end{array}$ & $\begin{array}{r}1500 \\
983\end{array}$ & 81 & 47.7 & 0.0539 & 0 & $\begin{array}{l}\text { Gray } \\
\text { Green }\end{array}$ \\
\hline 23X-CC, $10-12$ & 205.90 & 4.85 & 0.126 & 1.73 & 0.738 & 0.173 & 0.495 & 50.0 & 0.94 & 0.34 & 45 & 27 & 1500 & 34 & 89.1 & 0.0825 & $\mathrm{c}$ & $\begin{array}{l}\text { Ureen } \\
\text { White }\end{array}$ \\
\hline $24 \mathrm{X}-2,40-42$ & 209.40 & 35.6 & 0.644 & 12.8 & 3.99 & 0.0501 & 2.07 & 18.7 & 1.16 & 1.80 & 379 & 108 & 702 & 136 & 33.4 & 0.0571 & o & Green \\
\hline $24 \mathrm{X}-3,54-56$ & 211.02 & 26.4 & 0.844 & 9.15 & 4.48 & 0.141 & 2.07 & 24.6 & 1.51 & 2.26 & 179 & 63 & 889 & 193 & 44.0 & 0.105 & $\mathrm{v}$ & \\
\hline
\end{tabular}


Table 2 (continued).

\begin{tabular}{|c|c|c|c|c|c|c|c|c|c|c|c|c|c|c|c|c|c|c|}
\hline \multirow[b]{2}{*}{$\begin{array}{l}\text { Core, section, } \\
\text { interval }(\mathrm{cm})\end{array}$} & \multirow[b]{2}{*}{$\begin{array}{l}\text { Depth } \\
\text { (mbsf) }\end{array}$} & \multicolumn{9}{|c|}{ Major elements (wt\%) } & \multicolumn{4}{|c|}{ Trace elements $(\mu \mathrm{g} / \mathrm{g})$} & \multirow[b]{2}{*}{$\begin{array}{l}\mathrm{CaCO}_{3} \\
(\mathrm{wt} \%)\end{array}$} & \multirow[b]{2}{*}{$\mathrm{Ti} / \mathrm{Al}$} & & \\
\hline & & $\mathrm{SiO}_{2}$ & $\mathrm{TiO}_{2}$ & $\mathrm{Al}_{2} \mathrm{O}_{3}$ & $\mathrm{Fe}_{2} \mathrm{O}_{3}$ & $\mathrm{MnO}$ & $\mathrm{MgO}$ & $\mathrm{CaO}$ & $\mathrm{Na}_{2} \mathrm{O}$ & $\mathrm{K}_{2} \mathrm{O}$ & $\mathrm{Ba}$ & $\mathrm{Cr}$ & $\mathrm{Sr}$ & $\mathrm{Zr}$ & & & Group & Color \\
\hline $24 X-4,86-88$ & 212.83 & 29.3 & 0.983 & 9.91 & 4.63 & 0.178 & 2.23 & 24.1 & 1.59 & 2.24 & 228 & 55 & 860 & 218 & 43.1 & 0.112 & $\mathrm{v}$ & \\
\hline $24 X-5,15-17$ & 213.61 & 31.4 & 0.995 & 10.6 & 4.84 & 0.149 & 2.34 & 22.1 & 1.61 & 2.37 & 206 & 57 & 812 & 227 & 39.5 & 0.106 & $\mathrm{v}$ & Gray \\
\hline $24 \mathrm{X}-5,112-114$ & 214.58 & 16.8 & 0.454 & 6.09 & 2.49 & 0.231 & 1.20 & 37.7 & 1.12 & 1.47 & 171 & 38 & 1250 & 133 & 67.3 & 0.0846 & $\mathrm{v}$ & Pale gray \\
\hline $24 \mathrm{X}-6,17-19$ & 215.11 & 25.1 & 0.513 & 7.93 & 2.61 & 0.0810 & 1.67 & 30.6 & 0.97 & 1.84 & 270 & 55 & 1050 & 108 & 54.7 & 0.0733 & o & Gray \\
\hline $24 \mathrm{X}-6,39-41$ & 215.33 & 4.13 & 0.119 & 1.51 & 0.672 & 0.189 & 0.439 & 49.5 & 0.85 & 0.32 & $\begin{array}{l}33 \\
39\end{array}$ & 17 & 1440 & 30 & $\begin{array}{l}88.3 \\
878\end{array}$ & $\begin{array}{l}0.0893 \\
0.0867\end{array}$ & $\mathrm{c}$ & White \\
\hline $24 \mathrm{X}-6,71-73$ & 215.65 & 5.76 & 0.161 & 2.10 & 0.942 & 0.184 & 0.542 & 49.2 & 0.85 & 0.40 & 39 & 19 & 1460 & 39 & 87.8 & 0.0867 & c & White \\
\hline $24 \mathrm{X}-6,111-113$ & 216.05 & 19.1 & 0.694 & 6.96 & 3.21 & 0.111 & 1.46 & 33.8 & 1.36 & 1.50 & 125 & 38 & 1210 & 140 & 60.3 & 0.113 & $\mathrm{v}$ & Gray \\
\hline $24 \mathrm{X}-\mathrm{CC}, 20-22$ & 217.00 & 26.3 & 1.45 & 8.70 & 6.70 & 0.185 & 3.12 & 24.2 & 1.94 & 1.78 & 218 & 61 & 807 & 185 & 43.2 & 0.189 & $\mathrm{v}$ & Gray \\
\hline $25 \mathrm{X}-1,49-51$ & 217.62 & 34.9 & 0.625 & 13.0 & 3.81 & 0.0454 & 2.09 & 18.9 & 1.17 & 1.90 & 372 & 115 & 729 & 114 & 33.8 & 0.0546 & o & Green \\
\hline $25 \mathrm{X}-1,89-91$ & 218.02 & 35.4 & 0.645 & 13.6 & 4.56 & 0.0944 & 3.02 & 17.3 & 1.09 & 2.87 & 267 & 91 & 644 & 114 & 31.0 & 0.0539 & o & Gray \\
\hline $25 \mathrm{X}-1,123-125$ & 218.36 & 17.9 & 0.483 & 6.57 & 2.82 & 0.170 & 1.51 & 35.6 & 1.00 & 1.48 & 175 & 49 & 1210 & 101 & 63.6 & 0.0832 & $\mathrm{v}$ & \\
\hline $25 \mathrm{X}-1,146-148$ & 218.59 & 16.1 & 0.460 & 5.87 & 2.56 & 0.191 & 1.39 & 38.4 & 1.02 & 1.36 & 160 & 46 & 1290 & 99 & 68.5 & 0.0888 & $\mathrm{v}$ & Gray \\
\hline $25 \mathrm{X}-2,54-56$ & 219.17 & 16.5 & 0.576 & 5.61 & 3.02 & 0.139 & 1.60 & 37.9 & 1.02 & 1.27 & 139 & 44 & 1130 & 95 & 67.7 & $\begin{array}{l}0.117 \\
0.000\end{array}$ & $\mathrm{v}$ & Oray \\
\hline $25 \mathrm{X}-2,111-113$ & 219.74 & $\begin{array}{l}10.5 \\
15.7\end{array}$ & 0.567 & 5.26 & 2.88 & 0.141 & 1.56 & 38.2 & 0.96 & 1.13 & 149 & 41 & 1140 & 92 & $\begin{array}{l}68.1 \\
68.1\end{array}$ & 0.122 & $\mathrm{v}$ & \\
\hline $25 X-3,5-7$ & 220.18 & 15.9 & 0.577 & 5.32 & 2.74 & 0.143 & 1.56 & 37.4 & 1.06 & 1.19 & 165 & 44 & 1110 & 93 & 66.8 & 0.123 & $\mathrm{v}$ & Gray \\
\hline $25 \mathrm{X}-3,90-92$ & 221.03 & 19.5 & 0.358 & 7.40 & 2.48 & 0.0932 & 1.37 & 34.4 & 0.88 & 1.37 & 245 & 65 & 1260 & 75 & 61.4 & 0.0547 & o & Pale green \\
\hline $25 \mathrm{X}-4,9-11$ & 221.72 & 34.3 & 0.614 & 12.4 & 4.38 & 0.0729 & 2.62 & 19.3 & 1.12 & 2.70 & 275 & 80 & 711 & 115 & 34.4 & 0.0562 & o & File gieen \\
\hline $25 X-4,37-39$ & 222.00 & 33.1 & 0.590 & 12.0 & 4.26 & 0.0702 & 2.54 & 19.4 & 1.23 & 2.57 & 280 & 80 & 727 & 111 & 34.7 & 0.0555 & o & Gray \\
\hline $25 X-4,80-82$ & 222.43 & 33.6 & 0.568 & 11.8 & 4.21 & 0.0376 & 2.01 & 18.9 & 1.16 & 1.89 & 410 & 112 & 730 & 111 & 33.7 & 0.0544 & o & \\
\hline $25 \mathrm{X}-4,107-109$ & 222.70 & 31.7 & 0.535 & 11.1 & 7.96 & 0.0354 & 1.90 & 17.4 & 1.09 & 1.70 & 415 & 109 & 672 & 109 & 31.1 & 0.0547 & o & Dark green \\
\hline $25 X-5,13-15$ & 223.26 & 4.44 & 0.126 & 1.48 & 0.746 & 0.163 & 0.463 & 50.3 & 0.86 & 0.33 & 49 & 23 & 1530 & 29 & 89.7 & 0.0961 & $\mathrm{c}$ & \\
\hline $25 \mathrm{X}-5,30-32$ & 223.43 & 4.66 & 0.132 & 1.54 & 0.790 & 0.161 & 0.471 & 49.8 & 0.83 & 0.33 & 49 & 21 & 1520 & 29 & 88.8 & 0.0970 & $\mathrm{c}$ & White \\
\hline $25 \mathrm{X}-5,97-100$ & 224.10 & 41.2 & 0.738 & 15.7 & 5.31 & 0.0295 & 2.21 & 10.7 & 1.25 & 2.16 & 551 & 132 & 466 & 129 & 19.1 & 0.0532 & o & \\
\hline $25 \mathrm{X}-5,134-137$ & 224.47 & 41.0 & 0.728 & 15.3 & 6.13 & 0.0315 & 2.19 & 11.0 & 1.28 & 2.08 & 527 & 130 & 473 & 130 & 19.6 & 0.0540 & o & \\
\hline $25 \mathrm{X}-6,25-27$ & 224.88 & 40.5 & 0.707 & 14.6 & 6.80 & 0.0318 & 2.09 & 10.8 & 1.22 & 2.09 & 489 & 126 & 456 & 134 & 19.3 & 0.0548 & o & \\
\hline $25 \mathrm{X}-6,74-76$ & 225.37 & 41.3 & 0.726 & $\begin{array}{l}14.0 \\
15.2\end{array}$ & $\begin{array}{l}0.00 \\
5.33\end{array}$ & 0.0335 & 2.06 & $\begin{array}{l}11.0 \\
11.2\end{array}$ & 1.17 & 2.17 & 482 & 128 & 471 & 135 & 20.0 & 0.0543 & o & Dark green \\
\hline $25 \mathrm{X}-6,135-137$ & 225.98 & 34.8 & 0.626 & 13.2 & 4.50 & 0.0944 & 2.65 & 18.2 & 1.11 & 2.94 & 314 & 88 & 681 & 108 & 32.4 & 0.0537 & o & Gray/brown \\
\hline $25 \mathrm{X}-\mathrm{CC}, 29-31$ & 226.42 & 26.1 & 0.470 & 9.40 & 3.59 & 0.141 & 1.81 & 27.4 & 1.04 & 2.06 & 238 & 66 & 966 & 101 & 48.9 & 0.0567 & o & Gray/brown \\
\hline $26 \mathrm{X}-1,36-38$ & 227.05 & 25.7 & 0.463 & 9.11 & 3.59 & 0.127 & $\begin{array}{l}1.01 \\
1.74\end{array}$ & 28.7 & $\begin{array}{l}1.04 \\
1.05\end{array}$ & 2.12 & 247 & 62 & $\begin{array}{r}7020 \\
1020\end{array}$ & 101 & 51.3 & 0.0576 & 0 & \\
\hline $26 \mathrm{X}-1,121-123$ & 227.90 & 24.3 & 0.448 & 8.75 & 3.07 & 0.0962 & 1.65 & 29.8 & 1.03 & 1.92 & 258 & 61 & 1080 & 99 & 53.3 & 0.0580 & o & \\
\hline $26 \mathrm{X}-2,22-24$ & 228.41 & 24.8 & 0.457 & 8.99 & 3.13 & 0.101 & 1.67 & 30.2 & 1.04 & 1.94 & 256 & 60 & 1090 & 100 & 53.9 & 0.0577 & o & Pale gray \\
\hline $26 \mathrm{X}-2,141-143$ & 229.60 & 26.6 & 0.500 & 9.79 & 3.57 & 0.0974 & 2.65 & 26.2 & 0.97 & 2.45 & 256 & 68 & 901 & 86 & 46.8 & 0.0578 & o & \\
\hline $26 \mathrm{X}-3,17-19$ & 229.86 & 26.7 & 0.500 & 9.72 & 3.59 & 0.104 & 2.69 & 26.4 & 1.01 & 2.43 & 255 & 70 & 897 & 88 & 47.0 & 0.0583 & o & \\
\hline $26 \mathrm{X}-3,61-63$ & 230.30 & 26.9 & 0.502 & 9.68 & 3.68 & 0.114 & 2.71 & 26.3 & 1.00 & 2.44 & 252 & 69 & 892 & 89 & 46.9 & 0.0587 & o & Pale gray \\
\hline $26 \mathrm{X}-3,134-135$ & 231.02 & 37.9 & 0.688 & 14.2 & 4.87 & 0.0790 & 2.16 & 15.0 & 1.17 & 2.03 & 432 & 127 & 595 & 119 & 26.7 & 0.0549 & o & 1 ate gray \\
\hline $26 \mathrm{X}-3,140-141$ & 231.08 & 38.5 & 0.696 & 14.5 & 4.99 & 0.0755 & 2.20 & 14.3 & 1.18 & 2.04 & 414 & 130 & 572 & 124 & 25.5 & 0.0545 & o & \\
\hline $26 \mathrm{X}-3,146-147$ & 231.14 & 37.6 & 0.682 & 14.1 & 4.98 & 0.0728 & 2.14 & 15.0 & 1.19 & 2.00 & 416 & 129 & 597 & 124 & 26.7 & 0.0547 & o & Dark green \\
\hline $26 \mathrm{X}-4,79-81$ & 231.98 & 10.8 & 0.257 & 3.83 & 1.69 & 0.239 & 0.908 & 43.0 & 1.18 & 0.73 & 52 & 32 & 1240 & 47 & 76.8 & 0.0762 & $\mathrm{c}$ & White \\
\hline $26 \mathrm{X}-5,19-21$ & 232.88 & 34.0 & 0.611 & $\begin{array}{l}3.03 \\
13.0\end{array}$ & $\begin{array}{l}1.09 \\
5.43\end{array}$ & 0.206 & 2.60 & 18.7 & $\begin{array}{l}1.10 \\
1.10\end{array}$ & 3.05 & 270 & 84 & 678 & $\begin{array}{r}47 \\
105\end{array}$ & 33.4 & 0.0533 & o & White \\
\hline $26 \mathrm{X}-5,64-66$ & 233.33 & $\begin{array}{l}3.0 \\
29.6\end{array}$ & 0.543 & $\begin{array}{l}13.0 \\
11.4\end{array}$ & $\begin{array}{l}5.43 \\
3.69\end{array}$ & $\begin{array}{l}0.119 \\
0.119\end{array}$ & 2.30 & $\begin{array}{l}18.1 \\
23.6\end{array}$ & $\begin{array}{l}1.10 \\
1.01\end{array}$ & $\begin{array}{l}3.59 \\
2.59\end{array}$ & 295 & $\begin{array}{l}84 \\
74\end{array}$ & $\begin{array}{l}018 \\
829\end{array}$ & 95 & $\begin{array}{l}33.4 \\
42.2\end{array}$ & 0.0540 & ${ }_{0}^{\circ}$ & Pale brown \\
\hline $26 \mathrm{X}-5,145-147$ & 234.14 & 38.3 & 0.712 & $\begin{array}{l}11.4 \\
15.0\end{array}$ & 4.73 & 0.0850 & 2.47 & 14.3 & 1.22 & 3.29 & 348 & 88 & 553 & 138 & 25.6 & 0.0538 & o & 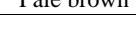 \\
\hline $26 \mathrm{X}-6,28-30$ & 234.47 & 38.0 & 0.710 & 14.9 & 5.14 & 0.0959 & 2.45 & 14.8 & 1.16 & 3.25 & 343 & $\begin{array}{l}80 \\
89\end{array}$ & 569 & $\begin{array}{l}130 \\
137\end{array}$ & 26.4 & 0.0541 & 0 & Green \\
\hline $26 \mathrm{X}-6,88-90$ & 235.07 & 51.4 & 0.956 & 19.1 & 7.53 & 0.103 & 3.36 & 1.09 & 1.47 & 4.29 & 346 & $\begin{array}{l}87 \\
109\end{array}$ & 151 & 191 & 1.94 & 0.0569 & o & Green \\
\hline $26 \mathrm{X}-\mathrm{CC}, 3-5$ & 235.72 & 37.6 & 0.693 & 14.0 & 4.77 & 0.101 & 2.47 & 15.0 & 1.32 & 3.03 & 349 & 83 & 558 & 142 & 26.7 & 0.0561 & o & \\
\hline $27 \mathrm{X}-1,12-14$ & 236.63 & 38.5 & 0.717 & 14.3 & 4.91 & 0.101 & 2.49 & 15.3 & 1.22 & 2.77 & 346 & 87 & 577 & 151 & 27.3 & 0.0567 & o & \\
\hline $27 \mathrm{X}-1,42-44$ & 236.92 & 37.9 & 0.712 & 14.5 & 4.99 & 0.123 & 2.49 & 15.2 & 1.20 & 2.91 & 328 & 88 & 576 & 150 & 27.1 & 0.0557 & o & Green \\
\hline $27 \mathrm{X}-1,103-105$ & 237.51 & 50.4 & 1.07 & 22.9 & 6.87 & 0.0355 & 1.74 & 0.67 & 1.30 & 2.13 & 470 & 160 & 136 & 179 & 1.20 & 0.0529 & o & \\
\hline $27 \mathrm{X}-1,135-137$ & 237.82 & 49.0 & 1.04 & 22.3 & 6.70 & 0.0344 & 1.68 & 1.67 & 1.25 & 2.11 & 462 & 156 & 169 & 176 & 2.98 & 0.0528 & o & Dark green \\
\hline $27 \mathrm{X}-2,21-23$ & 238.15 & 7.34 & 0.19 & 2.60 & 1.29 & 0.324 & 0.639 & 46.8 & 0.97 & 0.55 & 32 & 35 & 1430 & 42 & 83.5 & 0.0828 & $\mathrm{c}$ & White \\
\hline $27 \mathrm{X}-2,80-82$ & 238.73 & 39.6 & 0.731 & 15.5 & 6.35 & 0.404 & 2.87 & 12.2 & 1.23 & 3.05 & 414 & 96 & 479 & 132 & 21.7 & 0.0534 & o & $\begin{array}{l}\text { White } \\
\text { Brown }\end{array}$ \\
\hline $27 \mathrm{X}-3,14-16$ & 239.55 & 33.5 & 0.599 & 12.9 & 5.32 & 0.377 & 2.31 & 17.8 & 1.10 & 2.81 & 340 & 83 & 649 & 118 & 31.8 & 0.0527 & o & $\frac{\text { Brown }}{\text { Brown }}$ \\
\hline $27 \mathrm{X}-3,60-62$ & 239.99 & $\begin{array}{l}33.3 \\
37.2\end{array}$ & 0.644 & 13.5 & $\begin{array}{l}5.32 \\
4.64\end{array}$ & 0.0899 & 2.08 & $\begin{array}{l}1.8 \\
15.6\end{array}$ & 1.17 & $\begin{array}{l}2.81 \\
1.89\end{array}$ & $\begin{array}{l}340 \\
417\end{array}$ & $\begin{array}{r}83 \\
110\end{array}$ & $\begin{array}{l}049 \\
590\end{array}$ & 118 & $\begin{array}{l}37.8 \\
27.9\end{array}$ & 0.0540 & o & Brown \\
\hline $27 \mathrm{X}-3,98-100$ & 240.36 & 37.3 & 0.640 & 13.4 & 4.54 & 0.0822 & 2.07 & 15.8 & 1.19 & 1.88 & 397 & 110 & 595 & 122 & 28.2 & 0.0543 & o & Green \\
\hline $27 \mathrm{X}-3,134-136$ & 240.71 & 8.85 & 0.196 & 3.11 & 1.38 & 0.400 & 0.772 & 45.2 & 1.00 & 0.58 & 40 & 27 & 1330 & 38 & 80.7 & 0.0715 & $\mathrm{c}$ & White \\
\hline $27 \mathrm{X}-4,50-52$ & 241.36 & 32.0 & 0.600 & 11.8 & 5.31 & 0.277 & 3.05 & 21.8 & 1.03 & 2.80 & 321 & 84 & 70 & 102 & 38.9 & 0.0579 & o & $\begin{array}{l}\text { White } \\
\text { Brown }\end{array}$ \\
\hline $27 \mathrm{X}-4,110-112$ & 241.94 & 50.3 & 1.07 & 23.0 & 7.19 & 0.0404 & 1.55 & 0.26 & 1.24 & 2.34 & 256 & $\begin{array}{r}04 \\
140\end{array}$ & 102 & 195 & 0.46 & 0.0526 & o & Green \\
\hline $27 X-5,42-44$ & 242.75 & 50.8 & 1.10 & 22.8 & 6.07 & 0.0188 & 1.54 & 0.27 & 1.17 & 1.99 & 330 & 144 & 111 & 196 & 0.47 & 0.0545 & o & \\
\hline $27 X-5,72-74$ & 243.04 & 51.8 & 1.10 & 22.6 & 6.33 & 0.0300 & 1.57 & 1.30 & 1.18 & 2.03 & 316 & 143 & 147 & 202 & 2.33 & 0.0553 & o & \\
\hline $27 X-5,100-102$ & 243.31 & 50.1 & 1.06 & 22.0 & 6.19 & 0.0327 & 1.54 & 1.45 & 1.16 & 1.95 & 308 & 139 & 148 & 194 & 2.58 & 0.0549 & o & Dark green \\
\hline $27 \mathrm{X}-5,138-140$ & 243.68 & 41.6 & 0.696 & 14.5 & 5.48 & 0.0633 & 2.24 & 11.1 & 1.26 & 2.23 & 398 & 120 & 428 & 130 & 19.8 & 0.0544 & o & \\
\hline $27 X-6,7-9$ & 243.87 & 41.7 & 0.701 & 14.6 & 5.82 & 0.0611 & 2.24 & 11.2 & 1.24 & 2.11 & 378 & 123 & 432 & 130 & 20.0 & 0.0542 & o & Dark green \\
\hline $27 \mathrm{X}-6,120-122$ & 244.97 & 5.38 & 0.123 & 1.87 & 1.04 & 0.422 & 0.504 & 49.3 & 0.79 & 0.42 & 50 & 26 & 1440 & 37 & 88.0 & 0.0743 & $\mathrm{c}$ & White \\
\hline $27 \mathrm{X}-7,37-39$ & 245.62 & 43.30 & 0.720 & 15.7 & $\begin{array}{l}1.04 \\
5.98\end{array}$ & 0.0610 & 2.28 & 9.54 & 1.30 & 2.05 & 337 & 131 & 403 & 124 & $\begin{array}{l}\text { O. } \\
17.0\end{array}$ & 0.0519 & o & White \\
\hline $27 \mathrm{X}-\mathrm{CC}, 14-16$ & 245.85 & 43.1 & 0.706 & 15.3 & 6.00 & 0.0602 & 2.24 & 9.46 & 1.31 & 2.06 & 351 & 129 & 395 & 127 & 16.9 & 0.0523 & o & Dark green \\
\hline $28 \mathrm{X}-1,21-23$ & 246.22 & 40.3 & 0.661 & 14.0 & 5.50 & 0.0898 & 2.23 & 10.9 & 1.29 & 2.21 & 396 & 124 & 428 & 125 & 19.4 & 0.0535 & o & Dark greел! \\
\hline
\end{tabular}


Table 2 (continued).

\begin{tabular}{|c|c|c|c|c|c|c|c|c|c|c|c|c|c|c|c|c|c|c|}
\hline \multirow[b]{2}{*}{$\begin{array}{l}\text { Core, section, } \\
\text { interval }(\mathrm{cm})\end{array}$} & \multirow[b]{2}{*}{$\begin{array}{l}\text { Depth } \\
\text { (mbsf) }\end{array}$} & \multicolumn{9}{|c|}{ Major elements (wt\%) } & \multicolumn{4}{|c|}{ Trace elements $(\mu \mathrm{g} / \mathrm{g})$} & \multirow[b]{2}{*}{$\begin{array}{l}\mathrm{CaCO}_{3} \\
\text { (wt } \%)\end{array}$} & \multirow[b]{2}{*}{$\mathrm{Ti} / \mathrm{Al}$} & & \\
\hline & & $\mathrm{SiO}_{2}$ & $\mathrm{TiO}_{2}$ & $\mathrm{Al}_{2} \mathrm{O}_{3}$ & $\mathrm{Fe}_{2} \mathrm{O}_{3}$ & $\mathrm{MnO}$ & $\mathrm{MgO}$ & $\mathrm{CaO}$ & $\mathrm{Na}_{2} \mathrm{O}$ & $\mathrm{K}_{2} \mathrm{O}$ & $\mathrm{Ba}$ & $\mathrm{Cr}$ & $\mathrm{Sr}$ & $\mathrm{Zr}$ & & & Group & Color \\
\hline $28 \mathrm{X}-1,42-44$ & 246.43 & 39.4 & 0.649 & 13.9 & 6.16 & 0.0766 & 2.22 & 11.1 & 1.28 & 2.25 & 383 & 121 & 432 & 120 & 19.7 & 0.0531 & o & Dark green \\
\hline $28 \mathrm{X}-1,123-125$ & 247.24 & 37.0 & 0.707 & 14.2 & 4.64 & 0.131 & 2.43 & 15.9 & 1.11 & 2.93 & 364 & 84 & 596 & 143 & 28.3 & 0.0566 & o & \\
\hline $28 \mathrm{X}-2,11-13$ & 247.62 & 37.1 & 0.709 & 14.1 & 4.56 & 0.135 & 2.42 & 16.1 & 1.11 & 2.99 & 375 & 84 & 599 & 149 & 28.8 & 0.0570 & o & Dark green \\
\hline $28 \mathrm{X}-2,76-78$ & 248.27 & 40.1 & 0.637 & 13.6 & 5.24 & 0.111 & 2.20 & 13.7 & 1.23 & 2.21 & 397 & 120 & 552 & 120 & 24.5 & 0.0531 & o & Dark green \\
\hline $28 \mathrm{X}-3,10-12$ & 249.11 & 40.2 & 0.672 & 15.1 & 5.49 & 0.149 & 2.07 & 12.7 & 1.23 & 2.14 & 427 & 120 & 524 & 111 & 22.6 & 0.0506 & o & Dark green \\
\hline $28 \mathrm{X}-3,45-47$ & 249.46 & 40.3 & 0.668 & 14.2 & 5.61 & 0.104 & 2.21 & 12.2 & 1.20 & 2.10 & 387 & 132 & 488 & 123 & 21.8 & 0.0532 & o & Dark green \\
\hline $28 \mathrm{X}-\mathrm{CC}, 7-9$ & 249.64 & 41.4 & 0.662 & 13.9 & 5.49 & 0.112 & 2.18 & 12.4 & 1.24 & 2.18 & 349 & 133 & 489 & 132 & 22.0 & 0.0541 & o & Green \\
\hline $28 \mathrm{X}-\mathrm{CC}, 36-38$ & 249.93 & 40.3 & 0.690 & 14.7 & 5.69 & 0.157 & 2.20 & 12.1 & 1.22 & 2.29 & 356 & 132 & 492 & 119 & 21.5 & 0.0533 & o & Dark green \\
\hline $29 \mathrm{X}-1,88-90$ & 256.59 & 42.9 & 0.756 & 15.9 & 5.84 & 0.109 & 2.19 & 9.32 & 1.27 & 2.23 & 377 & 139 & 407 & 134 & 16.6 & 0.0540 & o & Dark green \\
\hline $29 \mathrm{X}-1,134-136$ & 257.05 & 48.3 & 0.984 & 22.2 & 6.77 & 0.0332 & 2.02 & 0.65 & 1.31 & 2.14 & 431 & 174 & 136 & 152 & 1.16 & 0.0503 & o & Dark green \\
\hline $29 \mathrm{X}-2,22-24$ & 257.43 & 41.2 & 0.795 & 16.2 & 6.36 & 0.309 & 2.46 & 11.5 & 1.15 & 3.32 & 388 & 93 & 505 & 142 & 20.5 & 0.0557 & o & Brown \\
\hline $29 \times-2,54-56$ & 257.75 & 5.03 & 0.124 & 1.76 & 0.963 & 0.448 & 0.488 & 49.0 & 0.84 & 0.33 & 88 & 19 & 1470 & 30 & 87.4 & 0.0801 & c & \\
\hline $29 \times-2,109-111$ & 258.30 & 5.19 & 0.143 & 1.77 & 0.858 & 0.340 & 0.58 & 48.4 & 1.17 & 0.38 & 85 & 17 & 1190 & 30 & 86.4 & 0.0919 & c & White \\
\hline $29 \times-3,34-36$ & 259.05 & 42.7 & 0.773 & 16.7 & 5.45 & 0.137 & 2.18 & 9.93 & 1.18 & 2.33 & 409 & 146 & 444 & 134 & 17.7 & 0.0524 & o & Dark green \\
\hline $29 \mathrm{X}-3,73-75$ & 259.44 & 7.01 & 0.185 & 2.46 & 1.05 & 0.564 & 0.600 & 47.2 & 0.99 & 0.46 & 49 & 23 & 1400 & 35 & 84.2 & 0.0850 & $\mathrm{c}$ & White \\
\hline $29 \mathrm{X}-3,146-148$ & 260.17 & 51.7 & 1.17 & 22.0 & 5.89 & 0.0561 & 1.91 & 0.64 & 1.13 & 2.14 & 326 & 165 & 141 & 201 & 1.14 & 0.0600 & o & Wark green \\
\hline $29 \mathrm{X}-4,36-38$ & 260.56 & 43.7 & 0.774 & 16.7 & 5.57 & 0.163 & 2.40 & 9.12 & 1.23 & 2.33 & 415 & 150 & 437 & 132 & 16.3 & 0.0524 & o & Dark green \\
\hline $29 \mathrm{X}-4,78-80$ & 260.98 & 49.8 & 0.965 & 21.5 & 6.74 & 0.0713 & 2.10 & 1.17 & 1.28 & 2.37 & 348 & 172 & 160 & 155 & 2.09 & 0.0510 & o & Dark green \\
\hline $29 \mathrm{X}-5,23-25$ & 261.93 & 51.3 & 1.10 & 23.5 & 5.91 & 0.0192 & 1.90 & 0.38 & 1.27 & 2.41 & 285 & 198 & 154 & 186 & 0.68 & 0.0531 & o & Dark green \\
\hline $29 \mathrm{X}-5,57-59$ & 262.27 & 49.5 & 0.920 & 19.8 & 6.64 & 0.225 & 2.65 & 1.35 & 1.26 & 2.56 & 543 & 187 & 173 & 152 & 2.41 & 0.0526 & o & Dark green \\
\hline $29 \mathrm{X}-5,116-118$ & 262.86 & 50.3 & 1.06 & 21.9 & 6.09 & 0.0690 & 1.96 & 1.59 & 1.17 & 2.25 & 319 & 168 & 180 & 184 & 2.84 & 0.0547 & o & Dark green \\
\hline $29 \mathrm{X}-5,140-142$ & 263.10 & 51.0 & 1.12 & 17.3 & 6.81 & 0.206 & 2.99 & 1.12 & 1.60 & 3.14 & 246 & 93 & 169 & 212 & 2.00 & 0.0730 & o & Dark brown \\
\hline $29 \mathrm{X}-6,27-29$ & 263.48 & 5.53 & 0.164 & 1.83 & 0.866 & 0.266 & 0.521 & 49.2 & 0.96 & 0.39 & 125 & 24 & 1400 & 36 & 87.8 & 0.102 & $\mathrm{c}$ & $\begin{array}{l}\text { Dark brown } \\
\text { White }\end{array}$ \\
\hline $29 \mathrm{X}-6,78-80$ & 263.99 & 5.36 & 0.148 & 1.75 & 0.942 & 0.285 & 0.500 & 49.0 & 0.93 & 0.32 & 163 & 21 & 1510 & 34 & 87.5 & 0.0956 & c & White \\
\hline $29 \mathrm{X}-6,131-133$ & 264.52 & 5.75 & 0.151 & 1.87 & 0.937 & 0.289 & 0.488 & 49.0 & 0.88 & 0.36 & 107 & 18 & 1570 & 33 & 87.5 & 0.0911 & c & White \\
\hline $29 \mathrm{X}-\mathrm{CC}, 28-30$ & 265.13 & 51.8 & 1.01 & 21.1 & 6.09 & 0.185 & 2.21 & 0.60 & 1.22 & 2.38 & 241 & 175 & 133 & 179 & 1.07 & 0.0541 & o & Dark gray \\
\hline $30 \times-1,25-27$ & 265.54 & 7.23 & 0.190 & 2.26 & 1.14 & 0.203 & 0.610 & 47.5 & 1.04 & 0.42 & 170 & 30 & 1490 & 40 & 84.8 & 0.0949 & $\mathrm{c}$ & White \\
\hline $30 \mathrm{X}-1,99-101$ & 266.26 & 44.6 & 0.749 & 15.3 & 5.56 & 0.0593 & 2.66 & 8.74 & 1.29 & 2.47 & 463 & 130 & 404 & 147 & 15.6 & 0.0553 & o & $\begin{array}{l}\text { Wnite } \\
\text { Green }\end{array}$ \\
\hline $30 \times-1,147-149$ & 266.74 & 50.0 & 0.913 & 20.4 & 6.08 & 0.0213 & 2.28 & 0.57 & 1.37 & 2.22 & 345 & 169 & 139 & 166 & 1.02 & 0.0508 & o & $\begin{array}{l}\text { Green } \\
\text { Dark green }\end{array}$ \\
\hline $30 \mathrm{X}-2,44-46$ & 267.21 & 39.7 & 0.661 & 13.5 & 4.94 & 0.0981 & 2.67 & 12.1 & 1.20 & 2.09 & 344 & 125 & 508 & 132 & 21.6 & 0.0553 & o & Dark green \\
\hline $30 X-2,115-117$ & 267.92 & 47.8 & 0.830 & 17.0 & 6.04 & 0.0509 & 2.79 & 5.51 & 1.46 & 2.23 & 341 & 143 & 300 & 162 & 9.84 & 0.0553 & o & Dark green \\
\hline $30 \mathrm{X}-2,144-146$ & 268.21 & 7.30 & 0.163 & 2.30 & 1.00 & 0.252 & 0.587 & 48.4 & 0.94 & 0.49 & 120 & 30 & 1520 & 44 & 86.4 & 0.0806 & $\mathrm{c}$ & White \\
\hline $30 \mathrm{X}-3,49-51$ & 268.76 & 32.9 & 0.660 & 9.72 & 3.32 & 0.0736 & 2.06 & 22.8 & 1.17 & 1.77 & 340 & 62 & 844 & 145 & 40.7 & 0.0770 & o & $\begin{array}{l}\text { White } \\
\text { Dark green }\end{array}$ \\
\hline $30 \mathrm{X}-3,118-120$ & 269.45 & 38.4 & 0.616 & 12.5 & 4.86 & 0.0622 & 2.73 & 14.9 & 1.27 & 1.95 & 443 & 109 & 620 & 125 & 26.6 & 0.0560 & o & Dark green \\
\hline $30 X-4,66-68$ & 270.43 & 33.4 & 0.725 & 12.5 & 4.20 & 0.0861 & 2.25 & 19.3 & 1.11 & 2.49 & 466 & 79 & 792 & 130 & 34.4 & 0.0656 & o & Daingitenti \\
\hline $30 X-4,115-117$ & 270.92 & 34.4 & 0.757 & 12.4 & 4.15 & 0.0902 & 2.26 & 19.3 & 1.17 & 2.35 & 428 & 83 & 782 & 137 & 34.4 & 0.0690 & o & Pale green \\
\hline $30 \times-5,77-79$ & 272.04 & 43.5 & 0.752 & 14.4 & 6.73 & 0.0499 & 2.82 & 7.90 & 1.35 & 1.88 & 437 & 128 & 345 & 153 & 14.1 & 0.0590 & o & Dark green \\
\hline $30 X-5,123-125$ & 272.50 & 46.1 & 0.714 & 14.1 & 6.05 & 0.0506 & 2.96 & 9.13 & 1.47 & 2.10 & 324 & 127 & 405 & 142 & 16.3 & 0.0575 & o & Dain gitenit \\
\hline $30 \mathrm{X}-6,20-22$ & 272.97 & 45.0 & 0.704 & 14.2 & 5.48 & 0.0486 & 2.96 & 9.14 & 1.48 & 2.14 & 309 & 127 & 403 & 136 & 16.3 & 0.0562 & o & Dark green \\
\hline $30 \mathrm{X}-6,73-75$ & 273.50 & 40.0 & 0.599 & 12.0 & 5.00 & 0.0649 & 2.67 & 14.0 & 1.32 & 1.94 & 405 & 113 & 569 & 129 & 25.0 & 0.0564 & o & \\
\hline $30 \mathrm{X}-\mathrm{CC}, 22-24$ & 274.17 & 41.0 & 0.586 & 11.6 & 5.05 & 0.0658 & 2.50 & 14.0 & 1.26 & 1.93 & 403 & 107 & 552 & 135 & 25.0 & 0.0571 & o & Dark green \\
\hline $31 \mathrm{X}-1,58-60$ & 275.49 & 43.2 & 0.700 & 14.4 & 5.10 & 0.0556 & 2.77 & 10.2 & 1.36 & 1.87 & 344 & 128 & 489 & 129 & 18.3 & 0.0552 & o & Dark green \\
\hline $31 \mathrm{X}-2,41-43$ & 276.82 & 52.8 & 0.953 & 18.1 & 5.79 & 0.0263 & 2.69 & 2.32 & 1.59 & 2.07 & 280 & 149 & 205 & 168 & 4.14 & 0.0595 & o & $\begin{array}{l}\text { Dark green } \\
\text { Dark green }\end{array}$ \\
\hline $31 \mathrm{X}-2,127-129$ & 277.68 & 36.8 & 0.513 & 10.6 & 4.38 & 0.0660 & 2.37 & 18.4 & 1.25 & 1.61 & 366 & 100 & 743 & 120 & 32.8 & 0.0550 & o & Dark gieen \\
\hline $31 \mathrm{X}-3,27-30$ & 278.19 & 37.8 & 0.497 & 10.1 & 3.92 & 0.0635 & 2.26 & 17.4 & 1.2 & 1. & 363 & 96 & 698 & 129 & 31.0 & 0.0556 & o & Gray/brown \\
\hline $31 \mathrm{X}-3,66-68$ & 278.5 & 5.68 & 0.143 & 1.69 & 0.693 & 0.271 & 0.485 & 49.1 & 0.9 & 0.34 & 157 & 26 & 1590 & 36 & 87.7 & 0.0963 & $\mathrm{c}$ & Uraypotion \\
\hline $31 \mathrm{X}-3,102-104$ & 278.94 & 7.77 & 0.206 & 2.32 & 0.860 & 0.256 & 0.597 & 46.8 & 1.00 & 0.46 & 141 & 30 & 1460 & 44 & 83.6 & 0.101 & $\mathrm{c}$ & White \\
\hline $31 \mathrm{X}-3,138-140$ & 279.30 & 47.1 & 0.721 & 14.1 & 6.50 & 0.126 & 3.14 & 8.04 & 1.83 & 1.68 & 236 & 128 & 425 & 141 & 14.4 & 0.0582 & o & Green \\
\hline $31 X-4,36-38$ & 279.78 & 7.41 & 0.171 & 2.16 & 0.816 & 0.299 & 0.561 & 48.4 & 0.96 & 0.47 & 137 & 30 & 1570 & 42 & 86.4 & 0.0896 & c & White \\
\hline $31 \mathrm{X}-4,108-110$ & 280.50 & 35.9 & 0.529 & 11.0 & 4.07 & 0.0828 & 2.60 & 18.3 & 1.29 & 1.57 & 355 & 109 & 736 & 112 & 32.6 & 0.0547 & o & Green/brown \\
\hline $31 \mathrm{X}-4,146-148$ & 280.88 & 39.3 & 0.584 & 11.7 & 4.47 & 0.0806 & 2.67 & 16.0 & 1.36 & 1.87 & 331 & 110 & 680 & 125 & 28.5 & 0.0566 & o & Uivecinorown \\
\hline $31 \mathrm{X}-5,30-32$ & 281.22 & 39.1 & 0.578 & 11.7 & 4.40 & 0.0798 & 2.66 & 15.8 & 1.30 & 1.82 & 305 & 103 & 679 & 122 & 28.3 & 0.0561 & o & Green \\
\hline $31 X-5,63-65$ & 281.55 & 6.32 & 0.136 & 1.90 & 0.715 & 0.379 & 0.807 & 48.8 & 0.94 & 0.34 & 116 & 32 & 1310 & 36 & 87.2 & 0.0812 & $\mathrm{c}$ & White \\
\hline $31 \mathrm{X}-\mathrm{CC}, 19-21$ & 282.40 & 35.8 & 0.480 & 10.2 & 4.13 & 0.0781 & 2.57 & 17.7 & 1.28 & 1.52 & 353 & 100 & 733 & 104 & 31.6 & 0.0531 & o & White \\
\hline $32 \mathrm{X}-1,16-18$ & 284.97 & 38.4 & 0.502 & 10.7 & 4.03 & 0.0803 & 2.70 & 18.4 & 1.42 & 1.62 & 339 & 106 & 763 & 112 & 32.9 & 0.0531 & o & Green \\
\hline $32 \mathrm{X}-1,108-110$ & 285.88 & 42.9 & 0.483 & 9.80 & 3.86 & 0.0658 & 2.57 & $\begin{array}{l}15.4 \\
15.1\end{array}$ & 1.42 & 1.51 & 435 & 101 & 621 & 116 & 27.0 & 0.0559 & o & Uinerin \\
\hline $32 \mathrm{X}-2,58-60$ & 286.86 & 43.4 & 0.468 & 9.43 & 4.19 & 0.0589 & 2.40 & 14.9 & 1.39 & 1.52 & 414 & 96 & 599 & 116 & 26.5 & 0.0562 & o & Green/brown \\
\hline $32 \mathrm{X}-2,123-125$ & 287.50 & 46.5 & 0.506 & 10.3 & 4.13 & 0.0667 & 2.54 & 12.5 & 1.44 & 1.58 & 410 & 101 & 514 & 120 & 22.3 & 0.0557 & o & G) \\
\hline $32 \mathrm{X}-3,8-10$ & 287.85 & 45.1 & 0.512 & 10.6 & 4.75 & 0.0596 & 2.60 & 12.5 & 1.4 & 1.65 & 405 & 104 & 521 & 114 & 22.2 & 0.0546 & o & Green/brown \\
\hline$-4,9-11$ & 289.33 & 54.2 & 0.822 & 16.7 & 5.63 & 0.0 & 3.14 & 1.98 & 1.74 & 1. & 448 & 148 & 206 & 14 & 3.53 & 0.0 & o & Green/brown \\
\hline 32 & 28 & 51.9 & 0.8 & 17.1 & 5. & & 3.21 & 0.75 & 1.96 & 1. & 296 & 179 & 170 & 162 & 1.33 & 0.0564 & o & Green/brown \\
\hline $32 \mathrm{X}-4,88-$ & 290.10 & 51.9 & 0.874 & 17.2 & 5.3 & 0.0271 & 2.99 & 2.82 & 1.75 & 1. & 219 & 138 & 238 & 151 & 5.04 & 0.0577 & o & Green \\
\hline $32 X-5,31-33$ & 291.04 & 49.2 & 0.506 & 10.1 & 3.97 & 0.0640 & 2.43 & 11.4 & 1.51 & 1.39 & 525 & 102 & 508 & 124 & 20.4 & 0.0570 & o & Uiver \\
\hline $32 X-5,104-106$ & 291.76 & 50.1 & 0.487 & 9.77 & 4.42 & 0.0472 & 2.31 & 10.9 & 1.45 & 1.46 & 480 & 95 & 481 & 126 & 19.5 & 0.0565 & o & Green/brown \\
\hline $32 \mathrm{X}-6,14-16$ & 292.34 & 9.97 & 0.218 & 2.79 & 1.25 & 0.145 & 0.729 & 45.7 & 0.89 & 0.54 & 194 & 35 & 1550 & 52 & 81.5 & 0.0885 & $\mathrm{c}$ & \\
\hline $32 \mathrm{X}-6,102-104$ & 293.20 & 46.3 & 0.546 & 11.3 & 4.52 & 0.0616 & 2.74 & 10.0 & 1.53 & 1.63 & 477 & 105 & 447 & 127 & 17.9 & 0.0549 & o & $\begin{array}{l}\text { White } \\
\text { Green/brown }\end{array}$ \\
\hline
\end{tabular}


Table 2 (continued).

\begin{tabular}{|c|c|c|c|c|c|c|c|c|c|c|c|c|c|c|c|c|c|c|}
\hline \multirow[b]{2}{*}{$\begin{array}{l}\text { Core, section, } \\
\text { interval }(\mathrm{cm})\end{array}$} & \multirow[b]{2}{*}{$\begin{array}{l}\text { Depth } \\
\text { (mbsf) }\end{array}$} & \multicolumn{9}{|c|}{ Major elements (wt\%) } & \multicolumn{4}{|c|}{ Trace elements $(\mu \mathrm{g} / \mathrm{g})$} & \multirow[b]{2}{*}{$\begin{array}{l}\mathrm{CaCO}_{3} \\
(\mathrm{wt} \%)\end{array}$} & \multirow[b]{2}{*}{$\mathrm{Ti} / \mathrm{Al}$} & \multirow[b]{2}{*}{ Group } & \multirow[b]{2}{*}{ Color } \\
\hline & & $\mathrm{SiO}_{2}$ & $\mathrm{TiO}_{2}$ & $\mathrm{Al}_{2} \mathrm{O}_{3}$ & $\mathrm{Fe}_{2} \mathrm{O}_{3}$ & $\mathrm{MnO}$ & $\mathrm{MgO}$ & $\mathrm{CaO}$ & $\mathrm{Na}_{2} \mathrm{O}$ & $\mathrm{K}_{2} \mathrm{O}$ & $\mathrm{Ba}$ & $\mathrm{Cr}$ & $\mathrm{Sr}$ & $\mathrm{Zr}$ & & & & \\
\hline $32 \mathrm{X}-\mathrm{CC}, 16-18$ & 294.26 & 50.3 & 0.675 & 13.6 & 5.72 & 0.0342 & 2.81 & 5.72 & 1.62 & 1.73 & 629 & 122 & 314 & 146 & 10.2 & 0.0565 & o & brown \\
\hline $33 \mathrm{X}-1,76-78$ & 295.08 & 53.4 & 0.786 & 16.5 & 5.73 & 0.0245 & 3.50 & 0.76 & 1.67 & 2.22 & 465 & 153 & 169 & 153 & 1.36 & 0.0541 & o & Dark gray \\
\hline $33 X-1,133-135$ & 295.63 & 53.4 & 0.796 & 16.2 & 5.91 & 0.0282 & 3.60 & 1.18 & 1.66 & 2.33 & 323 & 144 & 169 & 150 & 2.11 & 0.0556 & o & Dark green \\
\hline $33 \mathrm{X}-2,44-46$ & 296.31 & 53.6 & 0.703 & 15.2 & 6.07 & 0.0469 & 4.05 & 1.56 & 1.81 & 2.36 & 412 & 162 & 165 & 132 & 2.79 & 0.0524 & o & Dark green \\
\hline $33 \mathrm{X}-2,98-100$ & 296.84 & 34.7 & 0.702 & 10.9 & 4.09 & 0.0887 & 2.50 & 19.6 & 1.21 & 2.02 & 478 & 94 & 816 & 108 & 35.0 & 0.0731 & $\mathrm{v}$ & Dan ginti \\
\hline $33 \times-2,138-140$ & 297.23 & 35.0 & 0.773 & 10.9 & 4.14 & 0.0923 & 2.44 & 20.2 & 1.29 & 2.03 & 502 & 93 & 819 & 117 & 36.0 & 0.0808 & $\mathrm{v}$ & Gray \\
\hline $33 \mathrm{X}-3,62-64$ & 297.96 & 48.4 & 0.649 & 13.6 & 5.58 & 0.0528 & 3.56 & 7.40 & 1.54 & 2.19 & 409 & 138 & 341 & 125 & 13.2 & 0.0540 & o & Green/brown \\
\hline $33 \mathrm{X}-3,143-146$ & 298.75 & 54.5 & 0.736 & 15.4 & 6.00 & 0.0522 & 3.73 & 2.06 & 1.66 & 2.29 & 340 & 151 & 178 & 139 & 3.68 & 0.0542 & o & $\begin{array}{l}\text { Ureen/forown } \\
\text { Dark gray }\end{array}$ \\
\hline $33 \mathrm{X}-4,34-36$ & 299.15 & 52.2 & 0.786 & 16.5 & 5.60 & 0.0196 & 3.38 & 0.84 & 1.73 & 2.06 & 592 & 166 & 181 & 138 & 1.50 & 0.0541 & o & $\begin{array}{l}\text { Dark gray } \\
\text { Dark gray }\end{array}$ \\
\hline $33 X-4,56-58$ & 299.37 & 54.7 & 0.885 & 18.1 & 6.04 & 0.0277 & 3.43 & 1.15 & 1.63 & 2.18 & 359 & 163 & 196 & 150 & 2.05 & 0.0555 & o & Dark gray \\
\hline $33 \mathrm{X}-4,145-147$ & 300.24 & 53.8 & $\begin{array}{l}0.000 \\
1.28\end{array}$ & $\begin{array}{l}10.1 \\
15.0\end{array}$ & 8.50 & 0.104 & 3.55 & $\begin{array}{l}1.13 \\
1.42\end{array}$ & 1.80 & 2.71 & 365 & 124 & 182 & 158 & 2.53 & 0.0970 & $\mathrm{v}$ & $\begin{array}{l}\text { Dark gray } \\
\text { Gray }\end{array}$ \\
\hline $33 \mathrm{X}-5,55-57$ & 300.83 & 55.3 & 1.18 & 16.2 & $\begin{array}{l}0.00 \\
7.21\end{array}$ & 0.0408 & 3.41 & $\begin{array}{l}1.27 \\
1.27\end{array}$ & 1.77 & 2.86 & 259 & $\begin{array}{r}124 \\
99\end{array}$ & $\begin{array}{l}182 \\
188\end{array}$ & 196 & 2.27 & 0.0827 & $\mathrm{v}$ & $\begin{array}{l}\text { Gray } \\
\text { Gray }\end{array}$ \\
\hline $33 \mathrm{X}-5,127-129$ & 301.54 & 54.1 & 0.845 & 17.9 & 5.86 & 0.0213 & 3.25 & 1.01 & 1.52 & 2.06 & 721 & 154 & $\begin{array}{l}169 \\
\end{array}$ & 154 & 1.80 & 0.0536 & o & $\begin{array}{l}\text { Gray gray } \\
\text { Dark }\end{array}$ \\
\hline $33 \mathrm{X}-6,22-24$ & 301.98 & 52.7 & 0.979 & 19.7 & 5.62 & 0.0172 & 2.93 & 0.75 & 1.53 & 2.11 & 337 & 157 & 183 & 183 & 1.33 & 0.0563 & o & Dark gray \\
\hline $33 X-7,27-29$ & 303.51 & 50.8 & 1.21 & 16.4 & 6.64 & 0.0306 & 3.23 & 4.46 & 1.59 & 2.87 & 488 & 134 & 271 & 152 & 7.96 & 0.0837 & $\mathrm{v}$ & $\begin{array}{l}\text { Dark gray } \\
\text { Dark gray }\end{array}$ \\
\hline $33 \mathrm{X}-\mathrm{CC}, 28-30$ & 303.99 & 53.1 & 1.10 & 15.6 & 7.37 & 0.0478 & 3.55 & 1.21 & 1.73 & 2.35 & 213 & 146 & 169 & 159 & 2.16 & 0.0797 & $\mathrm{v}$ & Dark gray \\
\hline $34 \mathrm{X}-1,79-81$ & 304.82 & 43.6 & 0.997 & 13.9 & 5.51 & 0.0418 & 2.85 & 9.39 & 1.54 & 2.02 & 453 & 139 & 423 & 140 & 16.8 & 0.0814 & $\mathrm{v}$ & \\
\hline $34 \mathrm{X}-1,104-106$ & 305.07 & 43.3 & 0.783 & 13.3 & 5.15 & 0.0420 & 2.87 & 10.0 & 1.50 & 1.90 & 456 & 131 & 438 & 123 & 17.9 & 0.0666 & $\mathrm{v}$ & Dark gray \\
\hline $34 \mathrm{X}-2,16-18$ & 305.67 & 39.6 & 0.571 & 11.9 & 4.93 & 0.0369 & 2.92 & 14.0 & 1.50 & 1.42 & 534 & 138 & 491 & 100 & 25.0 & 0.0543 & o & Dark gray \\
\hline $34 \mathrm{X}-2,33-35$ & 305.84 & 33.3 & 0.564 & 9.62 & 3.94 & 0.0664 & 2.26 & 23.1 & 1.17 & 1.31 & 450 & 99 & 573 & 126 & 41.1 & 0.0664 & c & \\
\hline $34 \mathrm{X}-2,44-46$ & 305.95 & 15.8 & 0.359 & 4.59 & 2.07 & 0.173 & 1.45 & 38.4 & 0.78 & 0.73 & 176 & 55 & 658 & 77 & 68.5 & 0.0886 & c & \\
\hline $34 \mathrm{X}-2,60-62$ & 306.11 & 6.30 & 0.213 & 1.87 & 0.806 & 0.244 & 1.08 & 48.2 & 0.68 & 0.37 & 70 & 28 & 621 & 43 & 86.0 & 0.130 & c & \\
\hline $34 \mathrm{X}-2,95-97$ & 306.46 & 4.21 & 0.147 & 1.21 & 0.531 & 0.219 & 0.987 & $\begin{array}{l}40.2 \\
49.9\end{array}$ & 0.65 & 0.25 & 43 & 33 & 572 & 43 & 89.1 & 0.137 & c & \\
\hline $34 \mathrm{X}-\mathrm{CC}, 27-29$ & 306.78 & 2.49 & 0.0983 & 0.766 & 0.320 & 0.128 & 0.999 & 51.4 & 0.41 & 0.12 & 31 & 30 & 565 & 32 & 91.8 & 0.146 & c & \\
\hline $35 \times-1,71-73$ & 314.32 & 6.83 & 0.174 & 1.95 & 0.712 & 0.123 & 1.15 & 47.6 & 0.79 & 0.34 & 44 & 34 & 405 & 88 & 85.0 & 0.101 & c & White to brown \\
\hline $35 \mathrm{X}-\mathrm{CC}, 36-37$ & 314.80 & 58.8 & 1.08 & 5.47 & 2.33 & 0.538 & 1.20 & 13.5 & 1.12 & 1.08 & 292 & 123 & 260 & 421 & 24.1 & 0.223 & sst & White \\
\hline $36 \mathrm{X}-1,91-93$ & 324.05 & 0.85 & 0.0319 & 0.287 & 0.124 & 0.179 & 0.863 & 53.0 & 0.80 & 0.06 & 4 & 29 & 439 & 26 & 94.5 & 0.126 & $\mathrm{c}$ & $\begin{array}{l}\text { White } \\
\text { White }\end{array}$ \\
\hline
\end{tabular}

Notes: Total iron expressed as $\mathrm{Fe}_{2} \mathrm{O}_{3} ; \mathrm{CaCO}_{3}$ calculated from $\mathrm{CaO}$ data, assuming that all $\mathrm{Ca}$ is present as carbonate. Lithologic groups are: $\mathrm{c}=$ calcareous; $\mathrm{v}=$ volcanic; $\mathrm{o}=$ organic-rich and other turbidites; sst $=$ volcaniclastic sandstone. Italicized letters in the Color column are Quaternary turbidite identifiers of Weaver and Kuijpers (1983). De Lange et al. (1987), Weaver, Buckley et al, 1989, and Weaver, Thomson, et al., 1989; bed divisions are indicated by horizontal lines. 


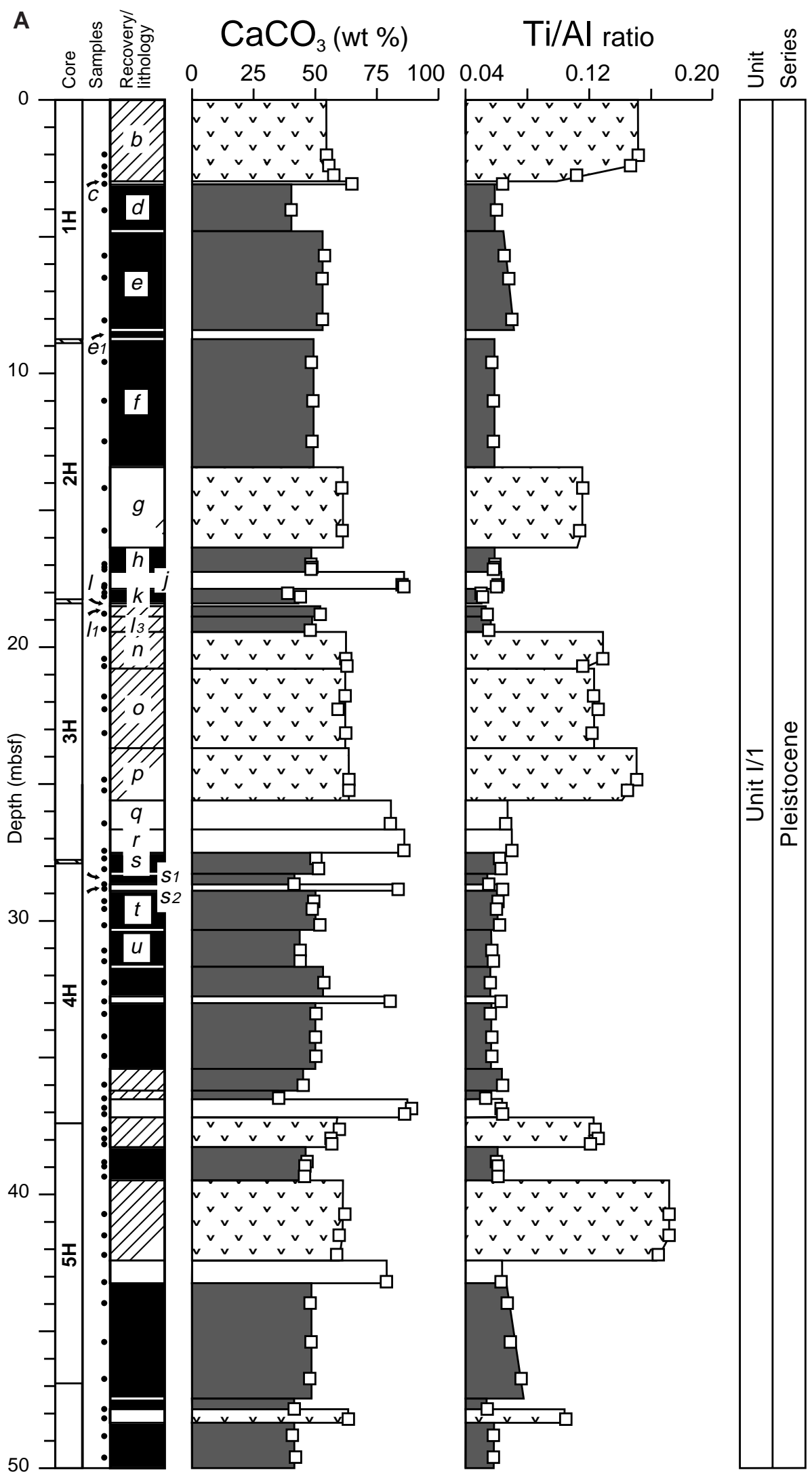

Figure 2. A-G. Carbonate and Ti/Al ratio profiles plotted against lithology for Site 950. Only turbidite data are plotted; pelagic intervals are excluded. Turbidite classification: (a) calcareous turbidites, $>75 \% \mathrm{CaCO}_{3}$ (calculated from $\mathrm{CaO}$ data); (b) volcanic turbidites, Ti/Al $>0.8$ and $\mathrm{CaCO}_{3}<75 \%$; (c) organic-rich and other turbidites. Turbidite lettering in Sections 157-950A-1H through 4H after Weaver and Kuijpers (1983) and Weaver et al. (1989a, 1989b); series boundaries derived from Howe and Sblendorio-Levy (Chap. 29, this volume). 


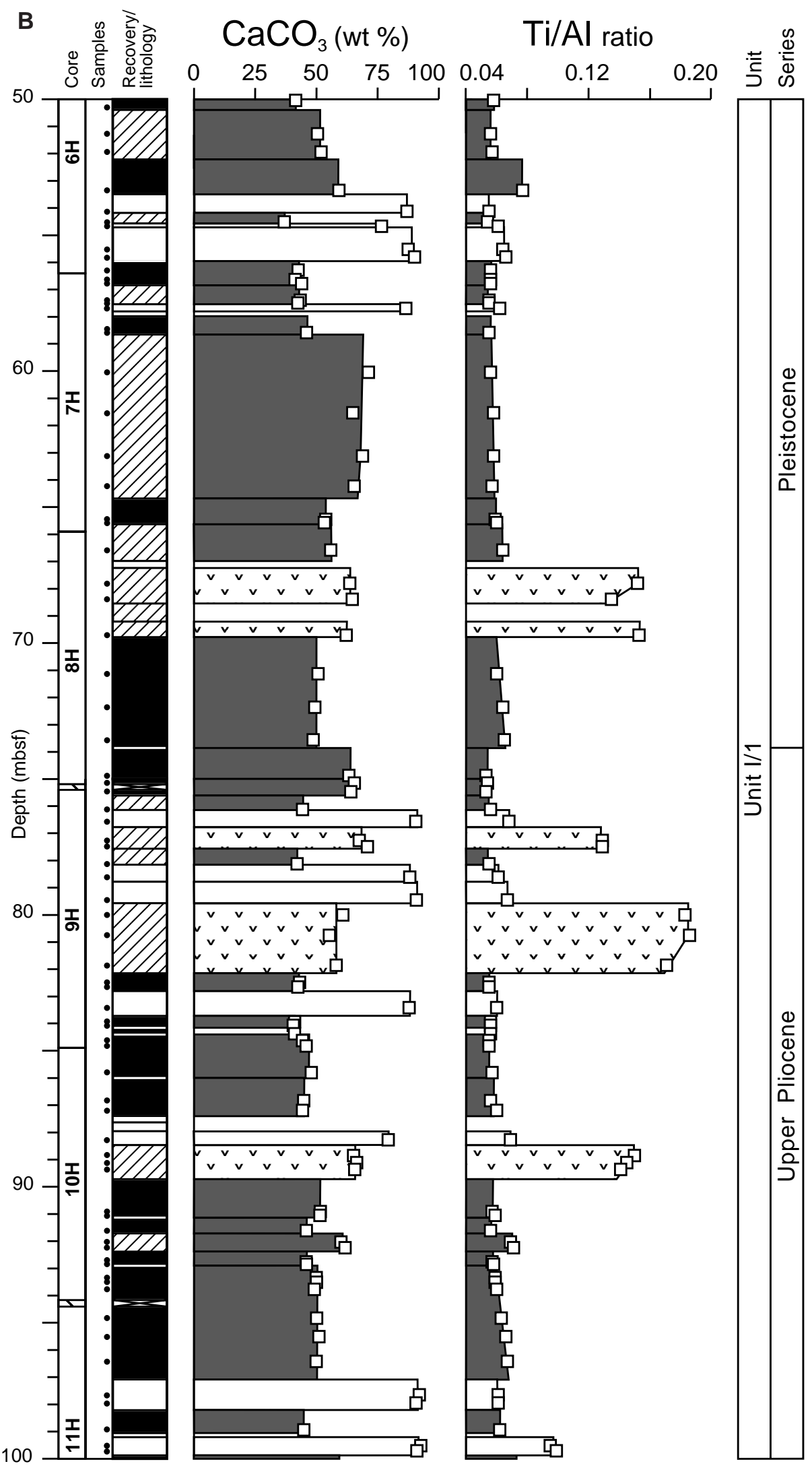

Figure 2 (continued). 


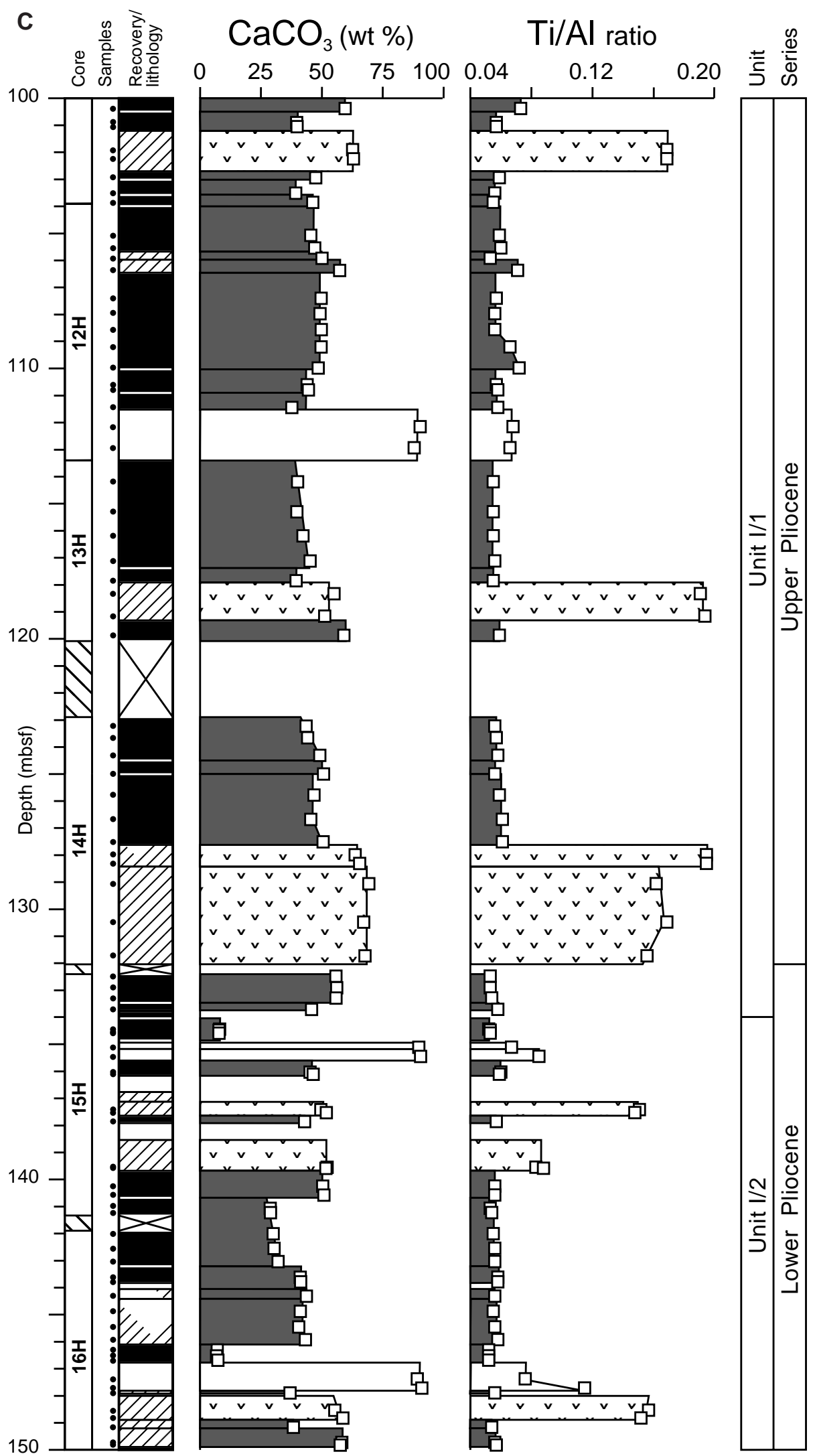

Figure 2 (continued). 


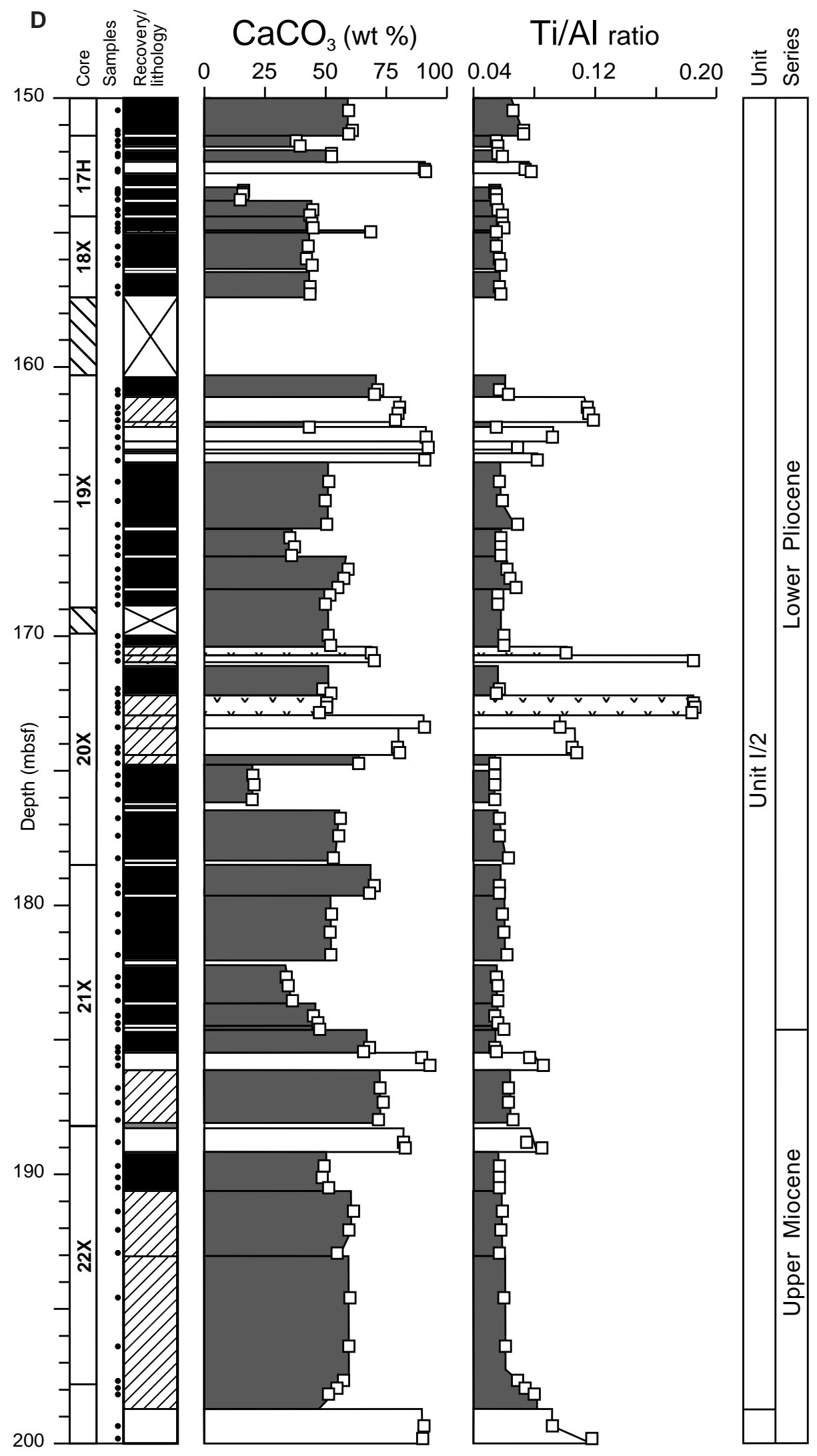

Figure 2 (continued). 


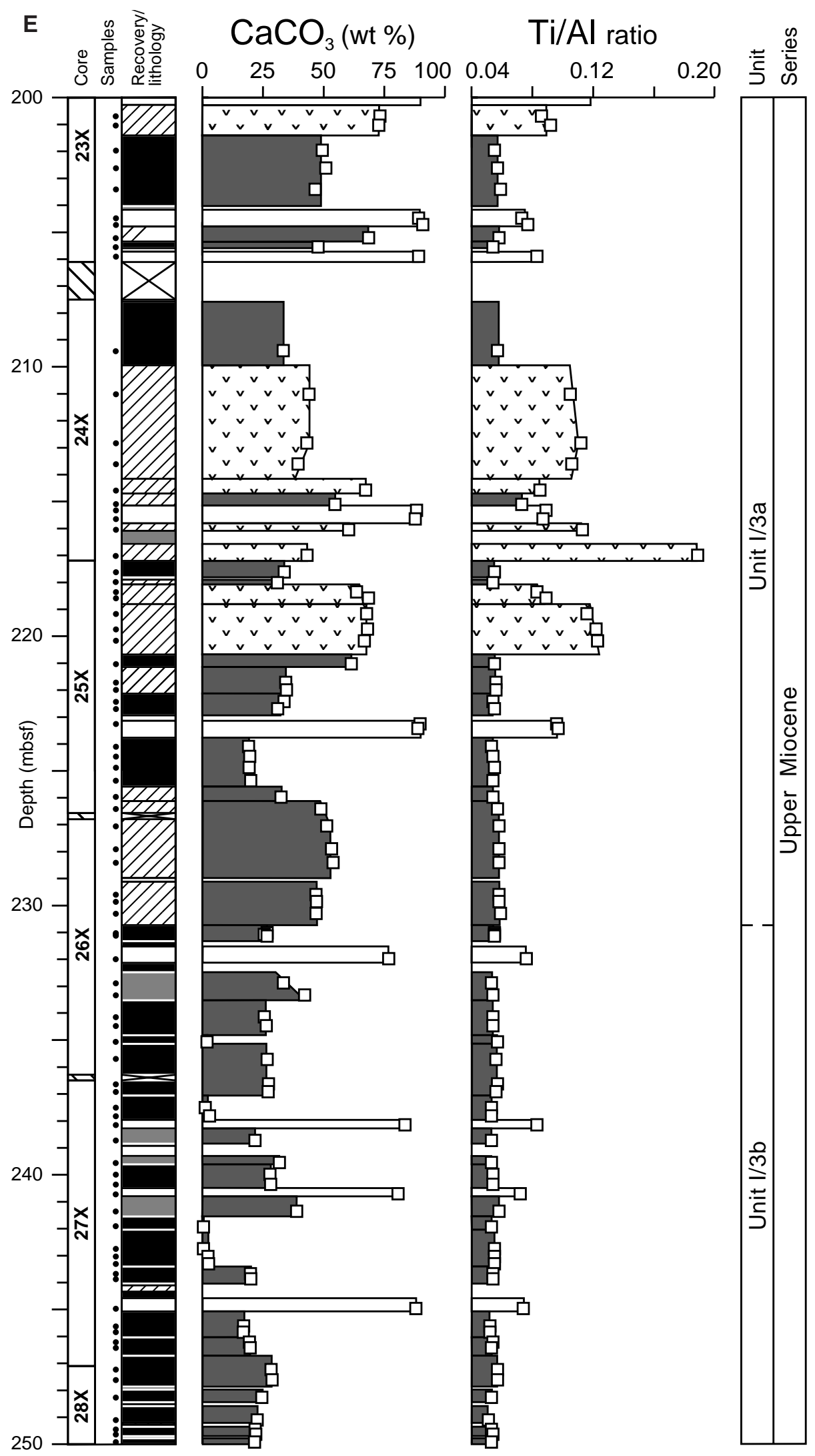

Figure 2 (continued). 


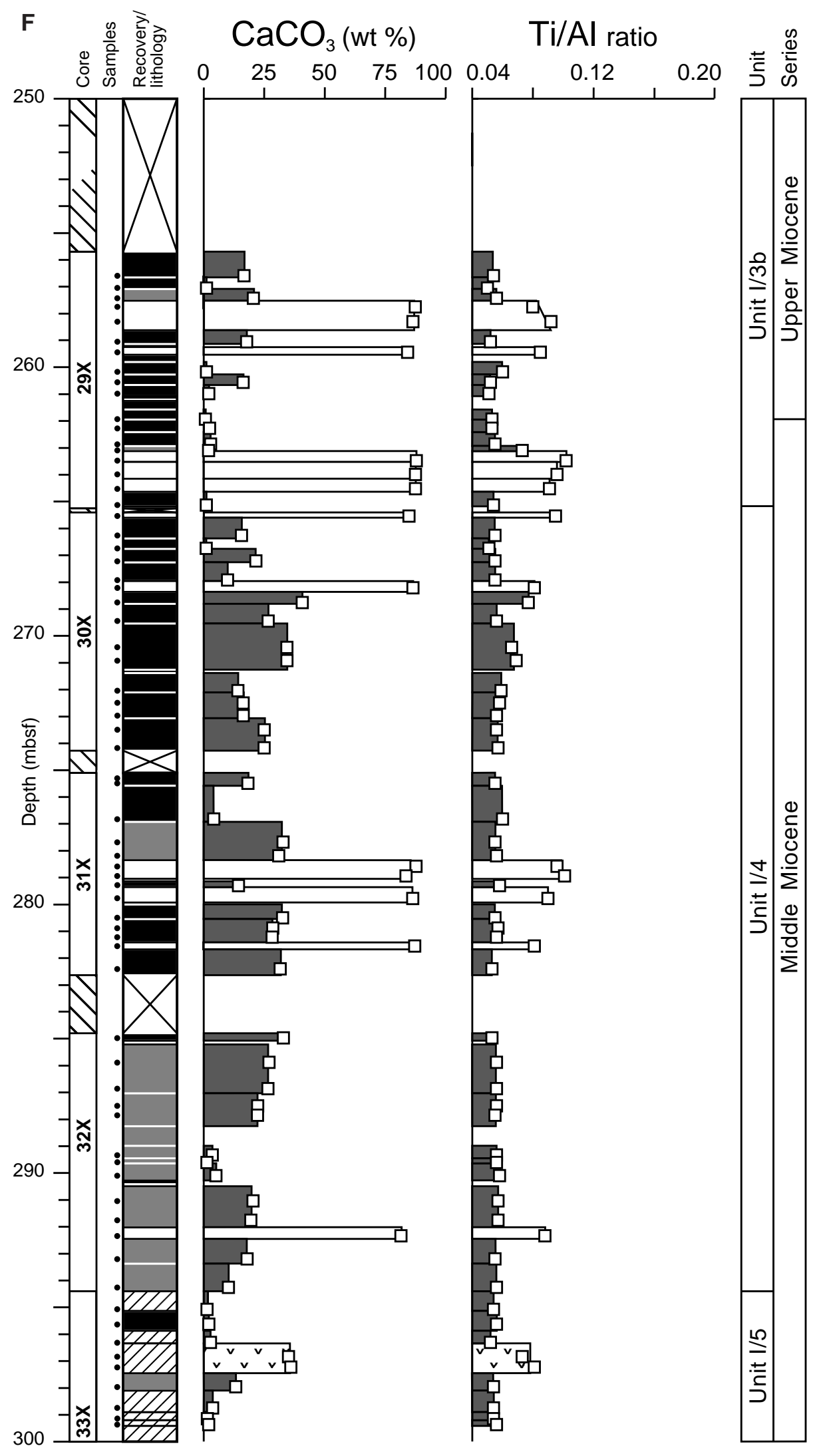

Figure 2 (continued). 


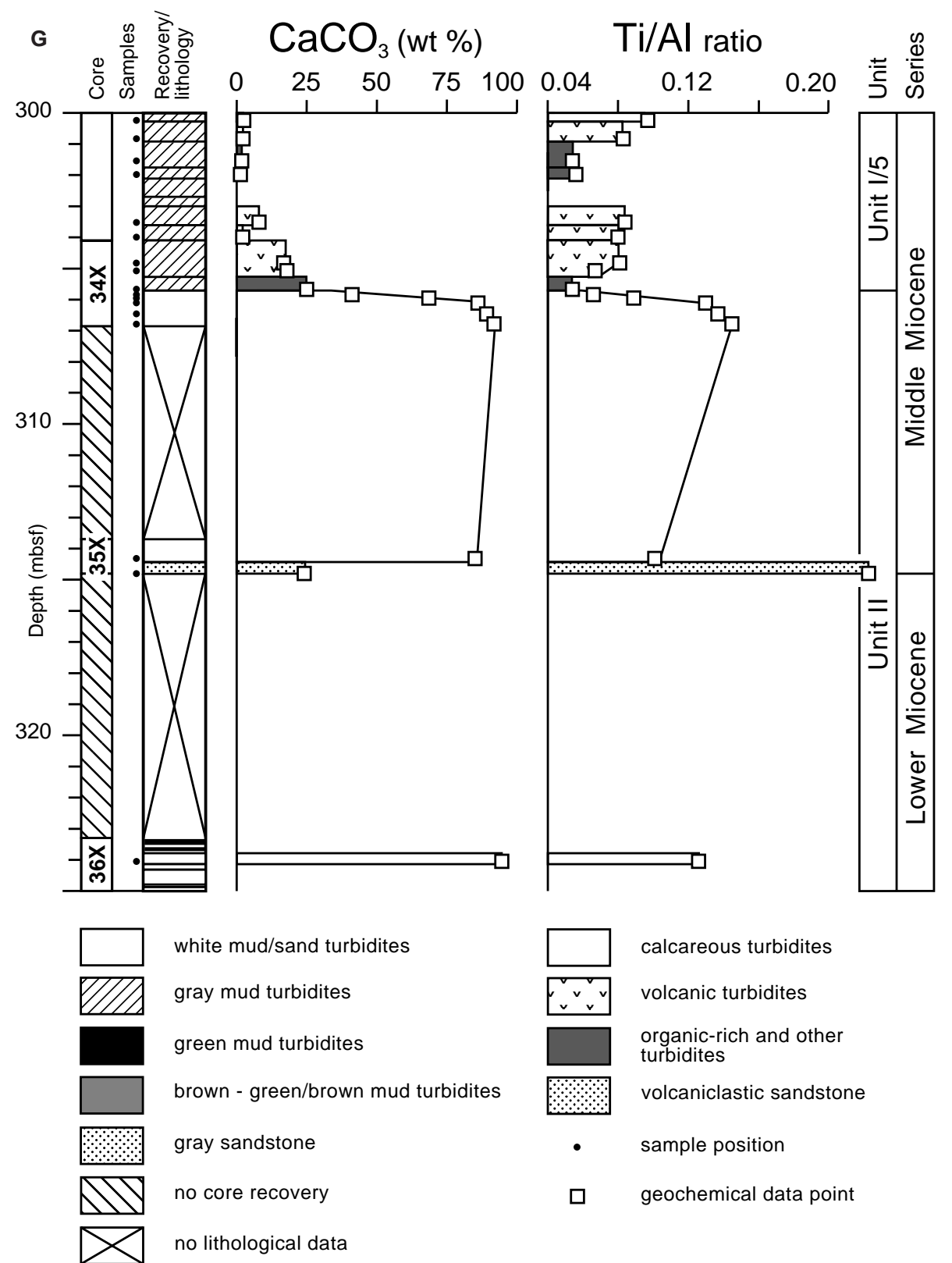

Figure 2 (continued).

Figure 2 demonstrates that shipboard identification of all gray turbidites as being members of the 'volcanic' group was erroneous, and that the frequency of volcanic turbidite deposition is much lower than originally thought. The detailed $\log$ (Fig. 2), combined with summary geochemical profiles (Fig. 3) for $\mathrm{CaCO}_{3}$ and Ti/Al ratios through shipboard lithostratigraphic Unit I (Schmincke, Weaver, Firth, et al., 1995), enable five new subdivisions to be designated here (defined here as chemostratigraphic Units I/1 through I/5):

\section{Unit I/1, 0-134 mbsf (upper Pliocene-Pleistocene)}

The pattern of turbidite deposition seen in the upper PleistoceneHolocene, continues down to $\sim 134$ mbsf (low-upper Pliocene). Turbidites in this interval fall clearly into one of the three previously defined turbidite groups. Ti/Al ratios for the volcanic turbidites form a broad array with increasing values downward, attaining maximum values of $\sim 0.2$ toward the base of the unit. There is a suggestion that the high-Ti (turbidites $b, p$; Fig. 2) and low-Ti $(g, o)$ subgroups defined by Pearce and Jarvis (1995) in the Quaternary sediments, continue downcore with a trend toward increasing Ti/Al ratios in both groups.

Carbonate contents of both the volcanic and calcareous turbidites also generally increase downward through this interval. $\mathrm{Ti} / \mathrm{Al}$ ratios of the organic-rich turbidites are remarkably consistent throughout the section, with small excursions toward higher values being caused by samples from the lower portions of beds, which commonly contain a proportion of entrained volcaniclastic grains (Pearce and Jarvis, 1992a, 1992b, 1995). A thick gray turbidite at 59-65 mbsf, originally assigned to the volcanic group (Schmincke, Weaver, Firth, et al., 1995), contains a high carbonate content of $68 \%$, but a low Ti/ Al ratio of 0.057 (Figs. 2B, 3). A sample (157-950A-7H-3, 78-79 $\mathrm{cm}$; $61.68 \mathrm{mbsf}$ ) from this turbidite analyzed for $\mathrm{C}_{\text {org }}$ aboard ship, 

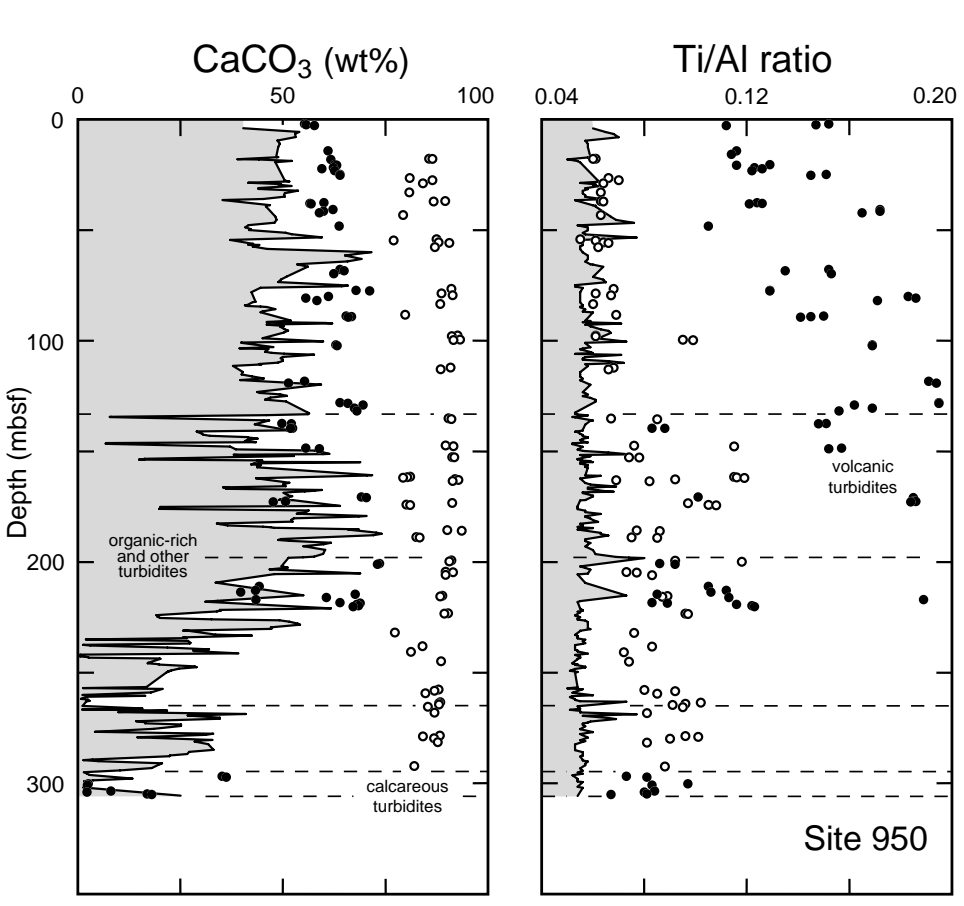

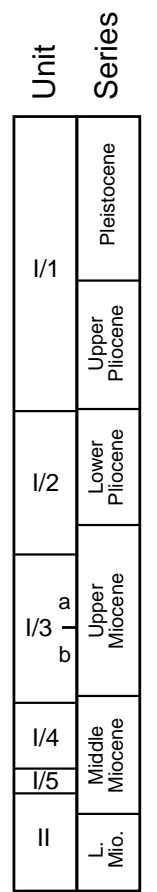

Figure 3. Geochemical variation in turbidite carbonate contents and Ti/Al ratios, Unit I, Site 950. Note the clear distinction between volcanic (solid circles; 71 data points), calcareous (open circles; 70 points), organic-rich and other (shaded area and continuous line; 338 analyses) turbidites in Unit I/1. yielded $0.51 \%$ (Schmincke, Weaver, Firth, et al., 1995), indicating that despite its color, the turbidite belongs to the organic-rich group.

The clear distinction between volcanic and other turbidites is shown by data for other elements known to be enriched in the volcaniclastic fraction (De Lange et al., 1987, 1989), particularly Fe/Al, $\mathrm{Cr} / \mathrm{Al}$, and $\mathrm{Zr} / \mathrm{Al}$ (Fig. 4). Fe/Al ratios, are also lowest in the organicrich turbidites, occur at intermediate levels in the calcareous turbidites, and are highest in the volcanic category. Chromium contents overlap between the organic-rich and volcanic groups, but trend to the highest values in the latter category. Zirconium contents are invariably highest in volcanic turbidites. Unlike $\mathrm{Ti}$, only $\mathrm{Cr} / \mathrm{Al}$ displays a clear downhole increase within the volcanic group of Unit $\mathrm{I} / 1$, and in this case a maximum is reached in a turbidite at $102 \mathrm{mbsf}$, some distance above the base of the unit.

Pearce and Jarvis (1995) recognized two subgroups within Quaternary organic-rich turbidites, based largely on potassium contents. Such a distinction is apparent in the $\mathrm{K} / \mathrm{Al}$ ratios of the green turbidites in Unit I/1 at Site 950 (Fig. 5), with high-K examples being characterized by ratios of $>0.3$, and a second subgroup having ratios of $0.2-$ 0.3 ; however, separation between the fields of the two subgroups becomes less clear in the mid-Pleistocene. A similar pattern is shown by $\mathrm{Mg} / \mathrm{Al}$ (Fig. 6), with organic-rich turbidites at 18 (turbidite $k$ ), 56, $84,85,115$, and $133 \mathrm{mbsf}$, in particular, displaying markedly $\mathrm{K}$ - and $\mathrm{Mg}$-depleted compositions. $\mathrm{Si} / \mathrm{Al}$ ratios vary considerably in the organic-rich turbidites of Unit I/1 (Fig. 7), but show no clear stratigraphic trends. Volcanic and calcareous turbidites display much less variation, with overlapping arrays on $\mathrm{K}, \mathrm{Mg}$, and Si plots, and little distinction between beds.

\section{Unit I/2, 134-199 mbsf (uppermost Miocene-lower Pliocene)}

A transition toward increased proportions of thin-bedded turbidites $\sim 134$ mbsf (Fig. 2C), is accompanied by the appearance of lowcarbonate $\left(<10 \% \mathrm{CaCO}_{3}\right)$ dark-green organic-rich turbidites, gray volcanic turbidites with low Ti/Al ratios $(\sim 0.09)$, and calcareous turbidites with high Ti/Al ratios of $>0.07$ (Fig. 3). Samples from calcareous turbidites with the highest $\mathrm{Ti} / \mathrm{Al}$ ratios (up to 0.12 ) are generally from the lower portions of beds. Together, these changes produce overlap between the calcareous and volcanic turbidite fields on the
Ti/Al ratio profile (Fig. 3), a characteristic that continues down through to the remainder of Unit I. Indeed, two gray calcareous turbidites within this interval (at 161 and $174 \mathrm{mbsf}$ ), have carbonate contents of $80 \%$, but high $\mathrm{Ti} / \mathrm{Al}$ ratios of $\sim 0.11$.

Despite the occurrence of a few dark green turbidites with very low carbonate contents, there is a general downward increase in the carbonate contents of the nonvolcanic turbidites through Unit I/2; $\mathrm{CaCO}_{3}$ contents attain a maximum in a series of three thick, gray turbidites with low Ti/Al ratios (Fig. 2D) at 199 mbsf. The bottom of Unit I/2 is defined by this shift in the carbonate profile (Fig. 3); calcareous turbidites also attain their highest $\mathrm{CaCO}_{3}$ contents of $>90 \%$ toward the base of the unit. Shipboard $\mathrm{C}_{\text {org }}$ determination (Schmincke, Weaver, Firth, et al., 1995) of a sample (157-950A$23 X-1,11-12 \mathrm{~cm} ; 197.91 \mathrm{mbsf})$ taken from one of the thick, gray nonvolcanic beds at the base of Unit I/2, gave a low value of $0.19 \%$. Given the considerable thickness $(>5 \mathrm{~m})$ of this turbidite, it is unlikely that it represents an oxidized member of the organic-rich group. This points to a different provenance for this, and possibly the other thick, gray nonvolcanic beds; additional organic carbon and other geochemical data are required to test this hypothesis.

A number of high-Ti volcanic turbidites continue to occur in Unit $\mathrm{I} / 2$, and also have high $\mathrm{Fe}$ and $\mathrm{Zr}$, but low $\mathrm{Cr}$ contents (Fig. 4). Occasional high Fe values in samples from organic-rich turbidites are attributed to the occurrence of scattered pyrite in the deeper turbidites. The two subgroups of organic-rich turbidites identified in Unit I/1 continue through Unit $\mathrm{I} / 2$, and display even clearer separation on $\mathrm{K}$ and $\mathrm{Mg}$ profiles (Figs. 5, 6). The low-carbonate organic-rich turbidites that first appear in Unit $\mathrm{I} / 2$ are also distinguished by having the lowest $\mathrm{K} / \mathrm{Al}(<0.2), \mathrm{Mg} / \mathrm{Al}$, and $\mathrm{Si} / \mathrm{Al}$ ratios in the sequence (Figs. 5 through 7), forming a distinctive third compositional subgroup on the geochemical profiles.

\section{Unit I/3, 199-265 mbsf (uppermost middle Miocene-upper Miocene)}

Turbidites below 199 mbsf generally display declining $\mathrm{CaCO}_{3}$ contents, reaching a minimum in a sequence of thin-bedded darkgreen turbidites at the base (Fig. 2E-G), which contains no significant carbonate. Even the calcareous turbidites become increasingly 

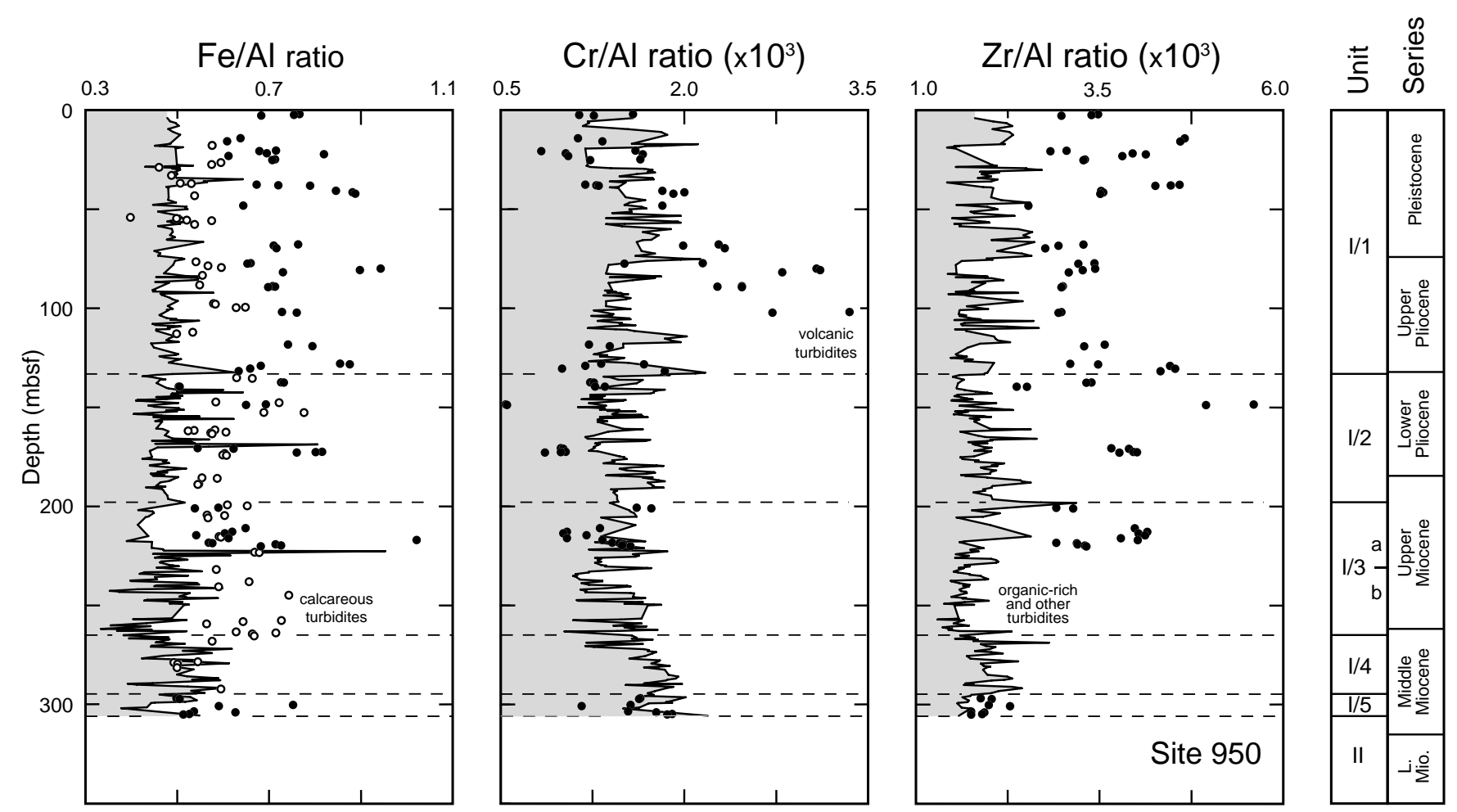

Figure 4. Geochemical variation in turbidite $\mathrm{Fe} / \mathrm{Al}, \mathrm{Cr} / \mathrm{Al}$, and $\mathrm{Zr} / \mathrm{Al}$ ratios, Unit I, Site 950. Volcanic (solid circles), calcareous (open circles), organic-rich and other (shaded area and continuous line) turbidites follow discrete trends similar to those displayed by Ti/Al ratio plots (Fig. 3). Calcareous turbidites have been omitted from the $\mathrm{Cr} / \mathrm{Al}$ and $\mathrm{Zr} / \mathrm{Al}$ plots because scattering produced by poor analytical reproducibility masks any geochemical trends.

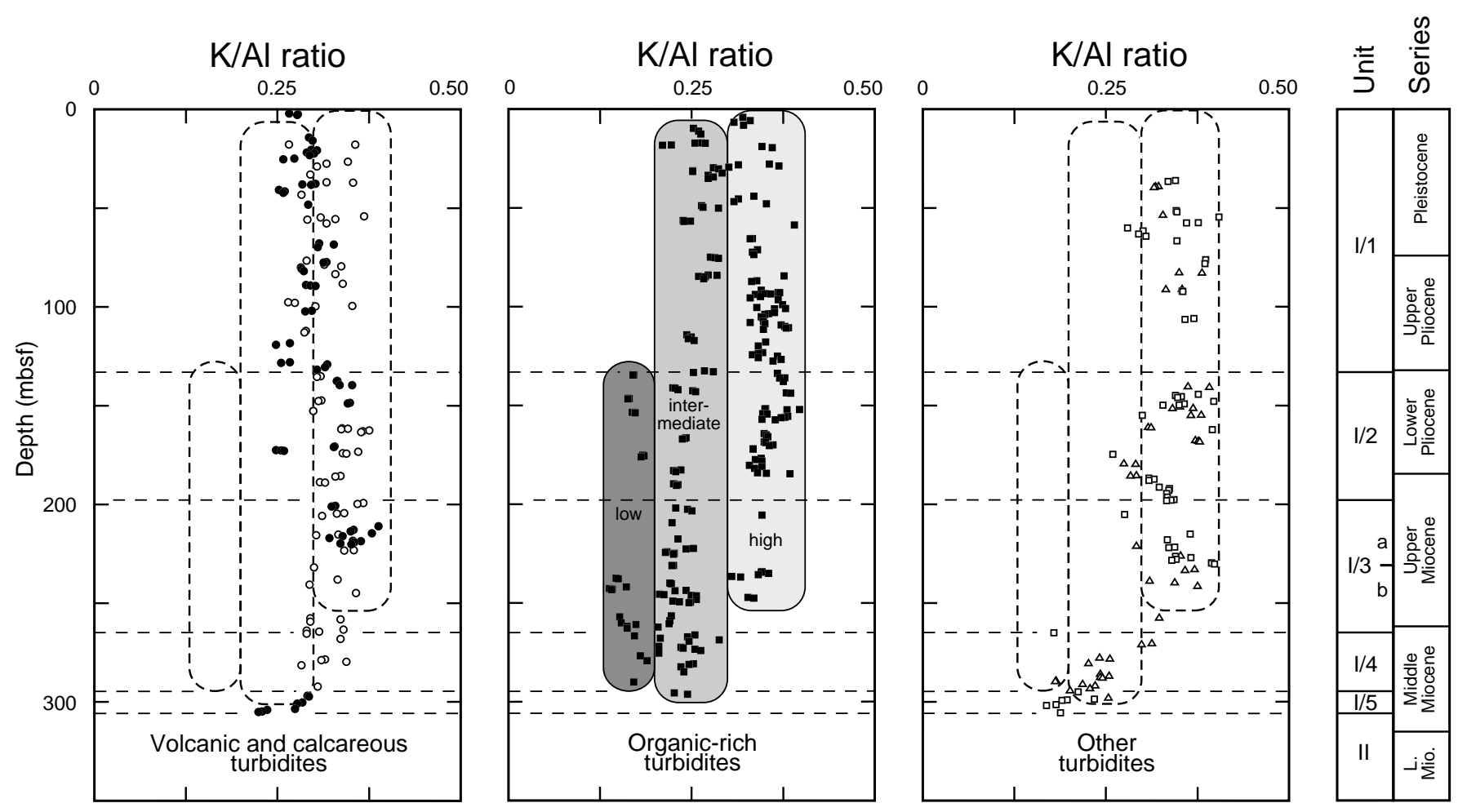

Figure 5. Stratigraphic variation in turbidite K/Al ratios, Unit I, Site 950. Volcanic (solid circles) and calcareous (open circles) turbidites have similar ratios, typically $>0.25$. Green organic-rich turbidites (filled squares; 229 analyses) display three distinct compositional arrays (shaded): with high ( $>0.3$ ), intermediate $(0.2-0.3)$ and low $(<0.2) \mathrm{K} / \mathrm{Al}$ ratios. Gray nonvolcanic (open squares; 60 data points) and other (open triangles; 48 analyses) turbidites fall mostly within the field (outlined) of the high K/Al organic-rich group, except near the base on Unit I. 

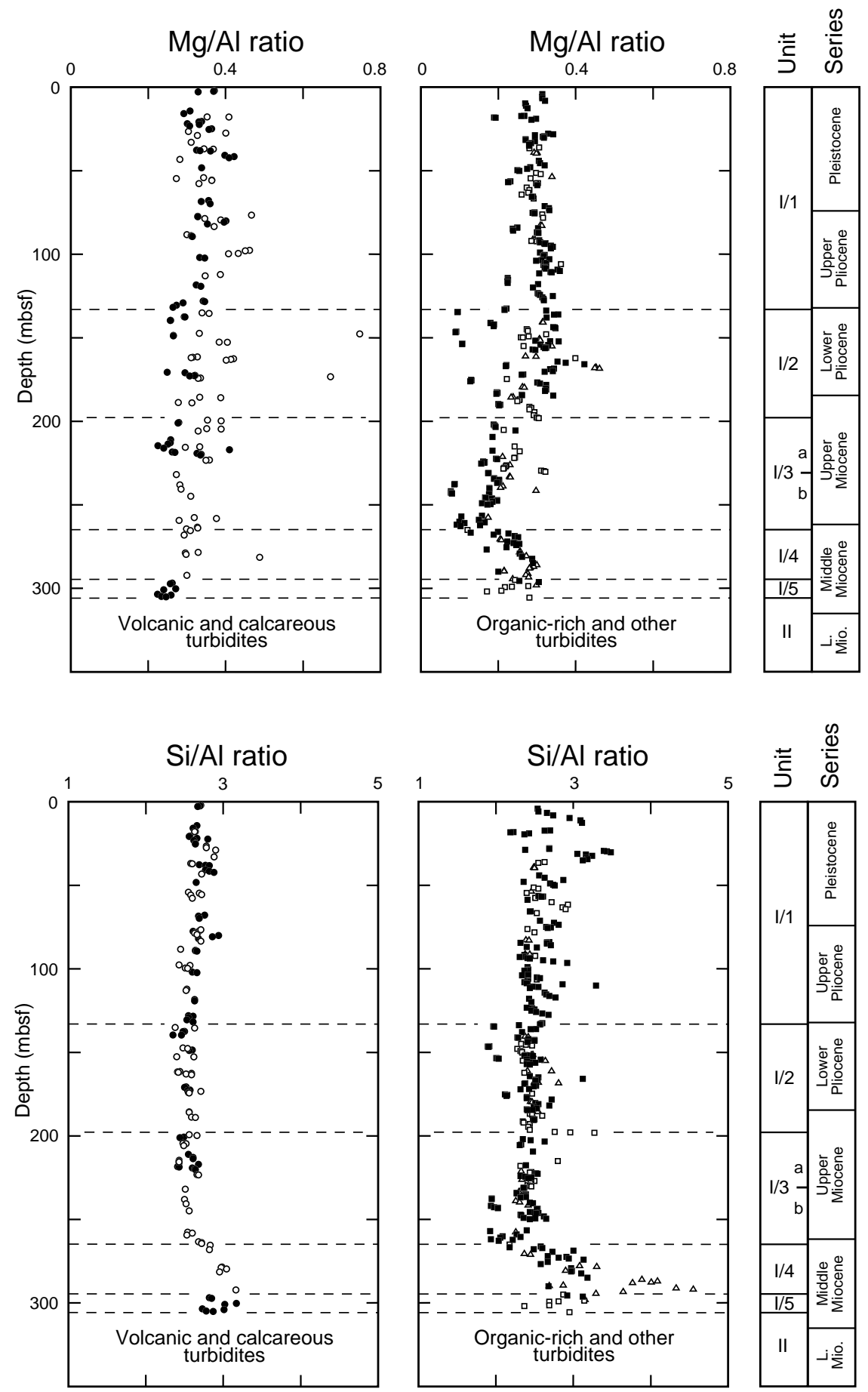

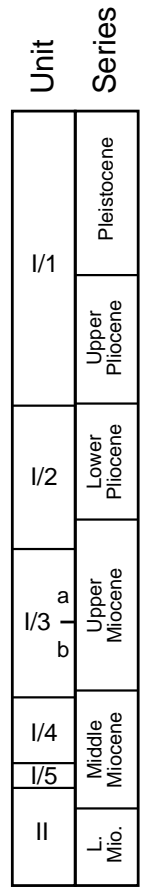

Figure 6. Stratigraphic variation in turbidite $\mathrm{Mg} / \mathrm{Al}$ ratios, Unit I, Site 950. Symbols as in Figure 5.

Figure 7. Stratigraphic variation in turbidite $\mathrm{Si} / \mathrm{Al}$ ratios, Unit I, Site 950. Symbols as in Figure 5. impure through this interval (Fig. 3). The last thick, gray volcanic turbidites occur $\sim 221 \mathrm{mbsf}$, and these, with one exception (at $217 \mathrm{mbsf}$ ), are characterized by low $\mathrm{Ti} / \mathrm{Al}$ ratios of $\leq 0.12$. There is a noticeable overall decrease in bed thickness below $231 \mathrm{mbsf}$ (Fig. 2E), with older turbidites typically $<2 \mathrm{~m}$ thick. This change in bedding style and lithology is used to subdivide the unit into I/3a (above) and I/3b (below).

Organic-rich turbidites in Unit I/3 display an increasing dominance of the intermediate $\mathrm{K}$ subgroup (K/Al ratios, 0.2-0.3) toward the base, with common low $\mathrm{K}(\mathrm{K} / \mathrm{Al}$ ratios, <0.2) examples in Subunit $\mathrm{I} / 3 \mathrm{~b}$ (Fig. 5). Separation between the subgroups is also seen on the $\mathrm{Mg} / \mathrm{Al}$ (Fig. 6), and to a lesser extent the Si/Al (Fig. 7) profiles.
However, there is a general shift toward lower Mg contents through Unit I/3, a consequence of declining carbonate, which contains significant $\mathrm{Mg}$. The $\mathrm{Mg} / \mathrm{Al}$ minimum, therefore, corresponds to the carbonate minimum at the base of Unit I/3; K and Si profiles remain unaffected.

\section{Unit I/4, 265-294 mbsf (mid- to high middle Miocene)}

Unit I/4 is dominated by thinly bedded green and green/brown turbidites (Fig. 2; Table 2), with low and variable $\mathrm{CaCO}_{3}$ contents of $0 \%$ to $34 \%$. Calcareous turbidites have moderate carbonate contents (87\%) and high Ti/Al ratios of $\sim 0.09$. The oldest dark-green high $\mathrm{K}$ 
organic-rich turbidite occurs toward the top of Unit I/4 at $247 \mathrm{mbsf}$ (Fig. 5), although brown turbidites with similar compositions occur below, at 257 and 263 mbsf.

All turbidite groups display increasing $\mathrm{Si} / \mathrm{Al}$ ratios downward through Unit $\mathrm{I} / 4$, with the highest $\mathrm{Si} / \mathrm{Al}$ ratios occurring in a unique suite of green/brown turbidites between 286 and 294 mbsf (Figs. 2, 7; Table 2), at the very base of the unit.

\section{Unit I/5, 294-306 mbsf (low middle Miocene)}

The oldest beds in Unit I consist of a thin-bedded sequence of dark-gray turbidites (Unit I/5; Fig. 2), with variable Ti/Al ratios and low carbonate contents. Many have Ti/Al ratios of $>0.08$, and so are assigned to the volcanic group, but they are lithologically and geochemically distinct from younger members of the group, which only appear above $221 \mathrm{mbsf}$, in the upper Miocene. Despite their Tienriched signatures (Fig. 3), these middle Miocene volcanic turbidites have lower $\mathrm{Zr}$ contents (Fig. 4), than other volcanic turbidites in the core; they are further distinguished by having low $\mathrm{Mg}$ and $\mathrm{K}$, and high Si to Al ratios. Organic-rich and other turbidites in this interval are all medium to low K/Al varieties (Fig. 5), with relatively high $\mathrm{Si}$ contents (Fig. 7) and no clear separation between subgroups. No calcareous turbidites occur in Unit I/5.

\section{Unit II, 306-333 mbsf (lower Miocene-lowest middle Miocene)}

Unit II is characterized by very poor core recovery; only a few sections of carbonate sand and gravel were obtained. Downhole logs indicate that three coarse-grained carbonate units occur in Unit II (Schmincke, Weaver, Firth, et al., 1995), and that its base coincides with the base of the nonrecovered interval (top of Core 157-950A$37 \mathrm{X}$ ). A unique feature in this sequence is the occurrence at its top of a hybrid turbidite, which grades upward from carbonate sands and gravels containing $90 \% \mathrm{CaCO}_{3}$, at its base (Fig. 2), to brown muds with $40 \% \mathrm{CaCO}_{3}$ at its summit. High Ti/Al ratios of 0.14 in the coarser lower part of the turbidite reflect a high proportion of volcaniclastic material, dominantly zeolitic vitric tuff and basaltic rock fragments with occasional glass shards and mineral crystals; this fraction is diluted by increasing proportions of clay minerals upward, and at the top of the bed the Ti/Al ratio is only 0.07 .

A sample of a thinner calcareous turbidite sand at $324 \mathrm{mbsf}$ (Section 157-950A-36X-1, 91-93 cm; Fig. 2G; Table 2) also has a very high $\mathrm{Ti} / \mathrm{Al}$ ratio of 0.13 , suggesting a similar source to the thick hybrid turbidite. A gray volcaniclastic sandstone at $315 \mathrm{mbsf}$ (Section 157-950A-35X-CC, 36-37 cm; Fig. 2G; Table 2), displays the highest $\mathrm{Ti} / \mathrm{Al}$ ratios measured, at 0.22 . Both of these sediments have moderate $\mathrm{Fe}$ but very high $\mathrm{Cr}$ and $\mathrm{Zr}$ contents. Very high $\mathrm{Mg} / \mathrm{Al}$ ratios characterize the carbonate sediments within this interval, attaining a value of 3.4 in the turbidite sand at 324 mbsf. The volcaniclastic sandstone, on the other hand, displays low Mg but high Si to Al ratios.

\section{Turbidite Provenance}

\section{Organic-Rich Turbidites}

The deposition of organic-rich turbidites at Site 950 began in the early middle Miocene $(\sim 15 \mathrm{Ma})$, with the first dark-green turbidite, now at 296 mbsf (two thin, green turbidites occur below this, $\sim 324$ mbsf, but these contain negligible $\mathrm{C}_{\text {org }}$ ). Organic-rich turbidites have dominated deposition on the MAP since that time.

Organic-rich turbidites display major changes in carbonate contents and other geochemical parameters through the sequence. Deposition on the plain during the middle Miocene was predominantly thin-bedded, low-carbonate sediments with low to medium $\mathrm{K} / \mathrm{Al}$ and high Si/Al ratios. Major changes occurred during the late Miocene
(6-11 Ma), with a progressive increase in bed thicknesses and carbonate contents, and a shift toward the deposition of turbidites containing higher $\mathrm{K} / \mathrm{Al}$ and $\mathrm{Mg} / \mathrm{Al}$ ratios. Carbonate contents generally fell slightly through the latest Miocene to latest early Pliocene (3.5$6 \mathrm{Ma})$, a trend accompanied by decreasing bed thicknesses and an increasing dominance of high-K over medium-K sediments, but with the continued intermittent influx of flows with very low carbonate, $\mathrm{K}$-depleted compositions. The low-K source switched off in the latest early Pliocene, and deposition during the last 3.5 m.y. has been remarkably uniform, with deposition being dominated by thick-bedded, high-carbonate, high-K organic-rich turbidites, accompanied by the occasional input of medium-K beds.

Preliminary interpretation of these trends is that, since the latest Miocene, organic-rich turbidites have originated predominantly from a chlorite- and illite-rich sediment source area on the northwest African continental slope off Morocco (Fig. 1), but with regular input of more kaolinitic sediment from a southerly source area, probably off Western Sahara. A third source, possibly a high-productivity area located even further to the south, was particularly active during the early late Miocene and mid-Pliocene. The less potassic sediments that dominate the middle and upper Miocene may be a consequence of the increased importance of the southerly source area, and/or climate change, promoting mineralogical changes in the northern source. Diagenetic processes are not considered to be a likely cause of the observed geochemical trends, because pore-water profiles (Schmincke, Weaver, Firth, et al., 1995) demonstrate minor uptake rather than the loss of potassium from deeper sediments at Site 950.

\section{Volcanic Turbidites}

Pearce and Jarvis (1995) concluded that during the late Quaternary, high-Ti volcanic turbidites on the MAP were derived from the younger western Canary Islands (Fig. 1), or possibly the basaltic complexes of Tenerife, whereas the low-Ti subgroup reflected a more fractionated volcanic source, and probably originated from the northern flanks of the central and eastern Canary Islands.

The oldest members of the volcanic turbidite group at Site 950 are confined to the early middle Miocene, $\sim 14-16 \mathrm{Ma}$. These thin-bedded, dark-gray turbidites are lithologically quite distinct from younger members of the group and have trace-element-depleted signatures. Their origin is currently uncertain.

The first thick-bedded, gray volcanic turbidites were deposited in the mid-late Miocene, $~ 6.5 \mathrm{Ma}$. This corresponds to volcanic hiatuses on Gran Canaria and Fuerteventura, and the early submarine stage of Tenerife (Schmincke, 1976, 1982, 1994). It has been demonstrated that Gran Canaria was supplying very little sediment to the deep ocean at that time (Schmincke, Weaver, Firth, et al., 1995). Low Ti, $\mathrm{Fe}$, and $\mathrm{Cr}$, and moderate $\mathrm{Zr}$ concentrations predominate in the late Miocene turbidites, pointing toward an evolved volcanic source area, probably the slopes of Lanzarote or Gomera. However, an increasing proportion of Ti-rich turbidites were deposited during the early Pliocene,

A major change in volcanic turbidite geochemistry occurred at the beginning of the late Pliocene, $\sim 3.5 \mathrm{Ma}$, with the disappearance of low-Ti sediments and an influx of common turbidites with very high $\mathrm{Ti} / \mathrm{Al}$ and $\mathrm{Fe} / \mathrm{Al}$ ratios. This points to a major shift toward a more basaltic source area. The island of La Palma was initiated around this time (Schmincke, 1994), while activity had ceased on Gomera and a volcanic hiatus was occurring on Tenerife. Gran Canaria was undergoing the development of a large stratocone, with the eruption of alkali basalt, trachyte, basanite, and phonolitic lavas and pyroclastics. It is speculated that the development of the La Palma Shield may have caused sediment instability on the slopes of the western Canary Islands, providing a significant sediment source at that time. 
There is a decline in $\mathrm{Ti} / \mathrm{Al}$ and $\mathrm{Fe} / \mathrm{Al}$ and a general increase in $\mathrm{Zr}$ / $\mathrm{Al}$ ratios through the subsequent $3.5 \mathrm{~m}$.y., indicating increasing input of sediment from areas having more evolved volcanic compositions. La Palma, Tenerife, and, more recently, Hierro, seem likely sources for the more basic material, with Gran Canaria, Lanzarote, and Fuerteventura providing a more fractionated volcaniclastic component.

\section{Calcareous Turbidites}

The deposition of thin calcareous turbidites on the MAP has occurred since at least the late Eocene. They occur regularly through the middle Miocene to Pleistocene record. Prior to the late Pliocene ( 3.5 $\mathrm{Ma})$, geochemical evidence indicates that calcareous turbidites entrained significant amounts of basaltic volcaniclastic debris, implying the active erosion of the westerly seamount chains (Fig. 1), which are believed to have acted as their source area. Younger turbidites contain little volcaniclastic material, indicating that the seamounts have been covered by a pelagic drape since that time.

\section{Other Turbidites}

A number of turbidites that do not fall clearly into any of the above groups have been observed at Site 950. Many of these are thin, gray turbidites that probably represent the now completely oxidized members of the organic-rich group. Such turbidites fall entirely within the geochemical compositional arrays defined by green turbidites. A smaller number of beds fall between the main arrays and may have mixed compositions. The source area of several thick, gray turbidites with nonvolcanic signatures remain uncertain, and the distinctive package of middle Miocene green/brown Si-rich turbidites also merits further investigation.

Thick, carbonate-debris flows and calcareous turbidites deposited during the early Miocene have distinctive trace-element-enriched geochemical signatures, reflecting the inclusion of a high proportion of basaltic and other volcaniclastic debris. In the absence of dolomite, high $\mathrm{Mg} / \mathrm{Al}$ ratios point to the inclusion of high-Mg calcite in the carbonate-sand fraction. The latter is consistent with a shallow-water origin for these sediments. They probably originated from the upper flanks of the Cruiser/Hyères/Great Meteor Seamount chain, which may have been emergent at that time.

\section{CONCLUSIONS}

Significant turbidite deposition on the MAP at Site 950 began in the early Miocene with the deposition of thin, carbonate-sand turbidites and three thick carbonate-debris flows. These beds have distinctive geochemical signatures, reflecting the incorporation of significant amounts of basaltic material and shallow-water carbonate grains derived from the erosion of seamounts lying to the west of the plain. The middle Miocene to Pleistocene was dominated by the deposition of distal mud turbidites, beginning at $\sim 15 \mathrm{Ma}$. Three major turbidite compositional groups (organic-rich, volcanic, and calcareous), originally defined in the Quaternary of the MAP, have been recognized in the older sedimentary record on the plain. Geochemical data define five chemostratigraphic units within the turbidite succession, reflecting sediment evolution in turbidite source areas and changes in provenance during the history of deposition on the plain.

Organic-rich turbidites dominate the sedimentary record, and become progressively more $\mathrm{K}$ and $\mathrm{Mg}$ rich with time. Three subgroups are evident from the geochemical data, indicating significant changes in sediment sources, particularly during the early late Miocene (10$11 \mathrm{Ma}$ ), with a shift toward more potassic- (illitic and chloritic) and carbonate-rich compositions, and during the latest early Pliocene $(\sim 3.5 \mathrm{Ma})$ with the final disappearance of very low-K (kaolinite- rich), carbonate-poor turbidites. The high-K subgroup probably originated principally from a northern source area on the upper continental slope off Morocco, whereas high-Al sediments were derived from the south, off Western Sahara. Climatic changes are also likely to have modified sediment mineralogy in the competing sources areas.

Volcanic turbidites are volumetrically the second most important sediment type through most of the sequence, although carbonate turbidites are more frequent. A thin package of thinly bedded dark gray volcanic turbidites with distinctive trace-element-depleted geochemical signatures was deposited during the early-middle Miocene (14$16 \mathrm{Ma})$. These are of uncertain affinity. The first typical thick-bedded, Ti-, Fe-, and $\mathrm{Zr}$-rich volcaniclastic turbidites were deposited on the MAP in the mid-late Miocene, $6.5 \mathrm{Ma}$, and probably originated from the Canary Island slopes of Lanzarote or Gomera. A major change to turbidites with very high $\mathrm{Ti}$ and $\mathrm{Fe}$ contents occurred around the beginning of the late Pliocene $(\sim 3.5 \mathrm{Ma})$, possibly associated with sediment failure during the early growth of La Palma. Younger volcanic turbidites display a clear trend toward progressively more fractionated volcanic sources since $3.5 \mathrm{Ma}$, although their wide range of trace-element compositions indicate continued supply from a variety of different source areas on the Canary Island slopes.

Thin-bedded calcareous turbidites occur throughout the sequence. They originated predominantly from the seamount chains to the west of the MAP and, up until the latest early Pliocene, incorporated a significant proportion of basaltic material derived from erosion of the exposed volcanic edifices. Since $\sim 3.5 \mathrm{Ma}$, these seamounts have been largely covered by a pelagic sediment drape, which now provides the main sediment source for calcareous turbidites on the plain.

\section{ACKNOWLEDGMENTS}

We thank the crew, marine technicians, and fellow scientists of Leg 157 for their help and enthusiasm. In particular, Sten Lindblom, Anne Pimmel, and Robert Kemp managed to survive the shipboard geochemical program. Erinn McCarty remained jovial despite our requests for difficult samples. Meryl Batchelder provided valuable technical assistance in the initial stages of this project. Constructive reviews were provided by Gert De Lange, Tim Pearce, Guy Rothwell, and Phil Weaver. We gratefully acknowledge support of this work through NERC ODP grant GST/02/1097.

\section{REFERENCES}

Bouma, A.H., 1962. Sedimentology of Some Flysch Deposits: A Graphic Approach to Facies Interpretation: Amsterdam (Elsevier).

Colley, S., and Thomson, J., 1985. Recurrent uranium relocations in distal turbidites emplaced in pelagic conditions. Geochim. Cosmochim. Acta, 49:2339-2348.

1992. Behavior and mobility of U-series radionuclides in Madeira Abyssal Plain turbidites over the past 750,000 years. Mar. Geol., 109:141-158.

Colley, S., Thomson, J., and Toole, J., 1989. Uranium relocations and derivation of quasi-isochrons for a turbidite/pelagic sequence in the Northeast Atlantic. Geochim. Cosmochim. Acta, 53:1223-1234.

Colley, S., Thomson, J., Wilson, T.R.S., and Higgs, N.C., 1984. Post-depositional migration of elements during diagenesis in brown clay and turbidite sequences in the North East Atlantic. Geochim. Cosmochim. Acta, 48:1223-1235

De Lange, G.J., 1992a. Distribution of exchangeable, fixed, organic and total nitrogen in interbedded turbiditic/pelagic sediments of the Madeira abyssal plain, eastern North Atlantic. Mar. Geol., 109:95-114.

, 1992b. Distribution of various extracted phosphorus compounds in the interbedded turbiditic/pelagic sediments of the Madeira Abyssal Plain, eastern North Atlantic. Mar. Geol., 109:115-139.

De Lange, G.J., Jarvis, I., and Kuijpers, A., 1987. Geochemical characteristics and provenance of late Quaternary sediments from the Madeira 
Abyssal Plain, North Atlantic. In Weaver, P.P.E., and Thomson, J. (Eds.), Geology and Geochemistry of Abyssal Plains. Geol. Soc. Spec. Publ. London, 31:147-165.

De Lange, G.J., Middelburg, J.J., Jarvis, I., and Kuijpers, A., 1989. Geochemical characteristics and provenance of late Quaternary sediments from the Madeira and southern Nares Abyssal Plains (North Atlantic). In Schuttenhelm, R.T.E., Auffret, G.A., Buckley, D.E., Cranston, R.E., Murray, C.N., Shephard, L.E., and Spijkstra, A.E. (Eds.), Geoscience Investigations of Two North Atlantic Abyssal Plain-The ESOPE International Expedition: Luxembourg (Commission of the European Community), 2:785-851.

Govindaraju, K., 1994. 1994 compilation of working values and sample description for 383 geostandards. Geostand. Newsl., 18 (spec. iss.)

Jarvis, I., 1992. Sample preparation for ICP-MS. In Jarvis, K.E., Gray, A.L., and Houk, R.S. (Eds.), Handbook of Inductively Coupled Plasma Mass Spectrometry: Glasgow (Blackie), 172-224.

Jarvis, I., and Higgs, N., 1987. Trace-element mobility during early diagenesis in distal turbidites: late Quaternary of the Madeira Abyssal Plain, N Atlantic. In Weaver, P.P.E., and Thomson J. (Eds.), Geology and Geochemistry of Abyssal Plains. Geol. Soc. Spec. Publ. London, 31:179214.

Jarvis, I., and Jarvis, K.E., 1992. Inductively coupled plasma-atomic emission spectrometry in exploration geochemistry. In Hall, G.E.M. (Ed.), J. Geochem. Expl., 44:139-200.

Jones, K.P.N., 1988. Studies of fine-grained, deep-sea sediments [Ph.D. thesis]. Univ. of Cambridge, UK.

Jones, K.P.N., McCave, I.N., and Weaver, P.P.E., 1992. Textural and dispersal patterns of thick mud turbidites from the Madeira Abyssal Plain. Mar. Geol., 107:149-173.

Kidd, R.B., Hunter, P.M., and Simm, R.W., 1987. Turbidity-current and debris-flow pathways to the Cape Verde Basin: status of long-range sidescan sonar (GLORIA) surveys. In Weaver, P.P.E., and Thomson, J. (Eds.), Geology and Geochemistry of Abyssal Plains. Geol. Soc. Spec. Publ. London, 31:33-48.

Kuijpers, A., and Weaver, P.P.E., 1985. Deep-sea turbidites from the northwest African continental margin. Dtsch. Hydrogr. Z., 38:147-164.

McArthur, J.M., Tyson, R.V., Thomson, J., and Mattey, D., 1992. Early diagenesis of marine organic matter: alteration of the carbon isotopic composition. Mar. Geol., 105:51-61.

McCave, I.N., and Jones, K.P.N., 1988. Deposition of ungraded muds from high-density non-turbulent turbidity currents. Nature, 333:250-252.

Middelburg, J.J., 1993. Turbidites provide a unique opportunity to study diagenetic processes. Geol. Mijnbouw, 72:15-21.

Middelburg, J.J., and De Lange, G.J., 1988. Geochemical characteristics as indicators of the provenance of Madeira Abyssal Plain turbidites: a statistical approach. Oceanol. Acta, 11:159-165.

Pearce, T.J., 1991. The geology, geochemistry, sedimentology and provenance of Late Quaternary turbidites, Madeira Abyssal Plain [Ph.D. thesis]. CNAA, Kingston Polytechnic.

Pearce, T.J., and Jarvis, I., 1992a. Applications of geochemical data to modelling sediment dispersal patterns in distal turbidites: Late Quaternary of the Madeira Abyssal Plain. J. Sediment. Petrol., 62:1112-1129.

, 1992b. Composition and provenance of turbidite sands: Late Quaternary, Madeira Abyssal Plain. Mar. Geol., 109:21-53.

1995. High-resolution chemostratigraphy of Quaternary distal turbidites: a case study of new methods for the analysis and correlation of barren sequences. In Dunay, R.E., and Hailwood, E.A. (Eds.), Non-biostratigraphical Methods of Dating and Correlation. Geol. Soc. Spec. Publ. London, 89:107-143.

Rothwell, R.G., Pearce, T.J., and Weaver, P.P.E., 1992. Late Quaternary evolution of the Madeira Abyssal Plain, Canary Basin, NE Atlantic. Basin Res., 4:103-131.

Schmincke, H.-U., 1976. The geology of the Canary Islands. In Kunkel, G. (Ed.), Biogeography and Ecology in the Canary Islands: The Hague (W. Junk), 67-184.

1982. Volcanic and chemical evolution of the Canary Islands. In von Rad, U., Hinz, K., Sarnthein, M., and Seibold, E. (Eds.), Geology of the Northwest African Continental Margin: Berlin (Springer), 273-306.

, 1994. Geological Field Guide: Gran Canaria (6th ed.): Kiel, Germany (Pluto Press).
Schmincke, H.-U., Weaver, P.P.E., Firth, J.V., et al., 1995. Proc. ODP, Init. Repts., 157: College Station, TX (Ocean Drilling Program).

Searle, R.C., 1987. Regional setting and geophysical characterization of the Great Meteor East area in the Madeira Abyssal Plain. In Weaver, P.P.E., and Thomson, J. (Eds.), Geology and Geochemistry of Abyssal Plains. Geol. Soc. Spec. Publ. London, 31:49-70.

Stow, D.A.V., and Shanmugam, G., 1980. Sequences of structures in finegrained turbidites: comparison of recent deep-sea and ancient flysch sediments. Sediment. Geol., 25:23-42.

Thomson, J., Colley, S., Higgs, N.C., Hydes, D.J., Wilson T.R.S., and Sorensen, J., 1987. Geochemical oxidation fronts in NE Atlantic distal turbidites and their effects in the sedimentary record. In Weaver, P.P.E., and Thomson, J. (Eds.), Geology and Geochemistry of Abyssal Plains. Geol. Soc. Spec. Publ. London, 31:167-177.

Thomson, J., Higgs, N.C., Croudace, I.W., Colley, S., and Hydes, D.J., 1993. Redox zonation of elements at an oxic/post-oxic boundary in deep-sea sediments. Geochim. Cosmochim. Acta, 57:579-595.

Thomson, J., Higgs, N.C., Jarvis, I., Hydes, D.J., Colley, S., and Wilson, T.R.S., 1986. The behaviour of manganese in Atlantic carbonate sediments. Geochim. Cosmochim. Acta, 50:1807-1818.

Totland, M.M., Jarvis, I., and Jarvis, K.E., 1992. An assessment of dissolution techniques for the analysis of geological samples by plasma spectrometry. Chem. Geol., 95:35-62.

Weaver, P.P.E., 1993. High resolution stratigraphy of marine Quaternary sequences. In Hailwood, E.A., and Kidd, R.B. (Eds.), High Resolution Stratigraphy. Geol. Soc. Spec. Publ. London, 70:137-153.

Weaver, P.P.E., Buckley, D.E., and Kuijpers, A., 1989. Geological investigations of ESOPE cores from the Madeira Abyssal Plain. In Schuttenhelm, R.T.E., Auffret, G.A., Buckley, D.E., Cranston, R.E., Murray, C.N., Shephard, L.E., and Spijkstra, A.E. (Eds.), Geoscience Investigations of Two North Atlantic Abyssal Plains: The ESOPE International Expedition: Luxembourg (Commission of the European Community), 1:535555.

Weaver, P.P.E., and Kuijpers, A., 1983. Climatic control of turbidite deposition on the Madeira Abyssal Plain. Nature, 306:360-363.

Weaver, P.P.E., Masson, D.G., and Kidd, R.B., 1994. Slumps, slides and turbidity currents: sea-level change and sedimentation in the Canary Basin. Geoscientist, 4:14-16.

Weaver, P.P.E., and Rothwell, R.G., 1987. Sedimentation on the Madeira Abyssal Plain over the last 300,000 years. In Weaver, P.P.E., and Thomson, J. (Eds.), Geology and Geochemistry of Abyssal Plains. Geol. Soc. Spec. Publ. London, 31:71-86.

Weaver, P.P.E., Rothwell, R.G., Ebbing, J., Gunn, D., and Hunter, P.M., 1992. Correlation, frequency of emplacement and source directions of megaturbidites on the Madeira Abyssal Plain. Mar. Geol., 109:1-20.

Weaver, P.P.E., Searle, R.C., and Kuijpers, A., 1986. Turbidite deposition and the origin of the Madeira Abyssal Plain. In Summerhayes, C.P., and Shackleton, N.J. (Eds.), North Atlantic Palaeoceanography. Spec. Publ. Geol. Soc. London, 21:131-143.

Weaver, P.P.E., and Thomson, J., 1993. Calculation erosion by deep-sea turbidity currents during initiation and flow. Nature, 364:136-138.

Weaver, P.P.E., Thomson, J., and Jarvis, I. 1989. The geology and geochemistry of Madeira Abyssal Plain sediments: a review. In Freeman, T.J. (Ed.), Advances in Underwater Technology, Ocean Science and Offshore Engineering (Vol. 18): London (Graham and Trotman), 51-78.

Wilson, T.R.S., Thomson, J., Colley, S., Hydes, D.J., Higgs, N.C., and Sorensen, J., 1985. Early organic diagenesis: the significance of progressive subsurface oxidation fronts in pelagic sediments. Geochim. Cosmochim. Acta., 49:811-822.

Wilson, T.R.S, Thomson, J., Hydes, D.J., Colley, S., Culkin, F., and Sørensen, J., 1986. Oxidation fronts in pelagic sediments: diagenetic formation of metal-rich layers. Science, 232:972-975.

Date of initial receipt: 8 July 1996

Date of acceptance: 8 January 1997

Ms 157SR-129 